\title{
Geometric constraints in dual F-theory and heterotic string compactifications
}

\author{
Lara B. Anderson ${ }^{a}$ and Washington Taylor ${ }^{b}$ \\ ${ }^{a}$ Department of Physics, Robeson Hall, 0435, Virginia Tech, \\ 850 West Campus Drive, Blacksburg, VA 24061, U.S.A. \\ ${ }^{b}$ Center for Theoretical Physics, Department of Physics, Massachusetts Institute of Technology, \\ 77 Massachusetts Avenue, Cambridge, MA 02139, U.S.A.
}

E-mail: lara.anderson@vt.edu, wati@mit.edu

ABSTRACT: We systematically analyze a broad class of dual heterotic and F-theory models that give four-dimensional supergravity theories, and compare the geometric constraints on the two sides of the duality. Specifically, we give a complete classification of models where the heterotic theory is compactified on a smooth Calabi-Yau threefold that is elliptically fibered with a single section and carries smooth irreducible vector bundles, and the dual F-theory model has a corresponding threefold base that has the form of a $\mathbb{P}^{1}$ bundle. We formulate simple conditions for the geometry on the F-theory side to support an elliptically fibered Calabi-Yau fourfold. We match these conditions with conditions for the existence of stable vector bundles on the heterotic side, and show that F-theory gives new insight into the conditions under which such bundles can be constructed. In particular, we find that many allowed F-theory models correspond to vector bundles on the heterotic side with exceptional structure groups, and determine a topological condition that is only satisfied for bundles of this type. We show that in many cases the F-theory geometry imposes a constraint on the extent to which the gauge group can be enhanced, corresponding to limits on the way in which the heterotic bundle can decompose. We explicitly construct all (4962) F-theory threefold bases for dual F-theory/heterotic constructions in the subset of models where the common twofold base surface is toric, and give both toric and non-toric examples of the general results.

KEYwords: F-Theory, Superstrings and Heterotic Strings, Superstring Vacua

ArXiv EPRINT: 1405.2074 


\section{Contents}

1 Introduction and overview 1

1.1 Introduction 1

1.2 Overview of main results 4

1.2.1 Classification and enumeration of models 5

1.2.2 Topological constraints on symmetries and spectra 5

$\begin{array}{lll}1.3 & \text { Outline } & 6\end{array}$

2 Lessons from heterotic/F-theory duality in higher dimensions $\quad 7$

$\begin{array}{lll}2.1 & \text { Heterotic/F-theory duality in eight dimensions } & 7\end{array}$

2.2 Heterotic/F-theory duality in six dimensions 8

2.2.1 Dual 6D geometries 8

$\begin{array}{ll}\text { 2.2.2 Geometric conditions on vacua } & 9\end{array}$

2.2.3 Moduli and the stable degeneration limit 9

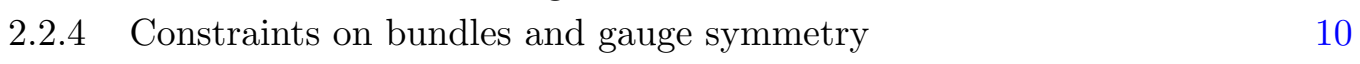

$\begin{array}{lll}\text { 2.2.5 Summary of } 6 \mathrm{D} \text { duality and relevance for 4D } & 14\end{array}$

3 Heterotic/F-theory duality in four dimensions $\quad \mathbf{1 5}$

$\begin{array}{lll}3.1 & \text { Geometry of heterotic/F-theory duality } & 15\end{array}$

$\begin{array}{lll}3.2 & \text { Possible base surfaces for smooth heterotic/F-theory duals } & 18\end{array}$

$\begin{array}{lll}3.3 & \text { Heterotic/F-theory duals with toric base surfaces } & 18\end{array}$

4 F-theory constraints $\quad 20$

4.1 Constraints on threefold base geometry 20

4.1.1 General constraints from F-theory geometry 21

$\begin{array}{lll}\text { 4.1.2 Constraints for toric bases } & 21\end{array}$

$\begin{array}{lll}\text { 4.1.3 } & \text { F-theory bounds on twists } & 22\end{array}$

4.1.4 Toric bounds on twists 23

4.1.5 General constraints on geometry from $f, g$ on divisors 24

4.1.6 General constraints on geometry from $f, g$ on curves 26

$\begin{array}{lll}4.2 & \text { Constraints on gauge enhancement } & 27\end{array}$

4.2.1 Constraints on $\mathrm{SU}(2)$ and $\mathrm{SU}(3)$ enhancement 28

4.2.2 Constraints from codimension two loci 28

5 Heterotic constraints $\quad 29$

5.1 The Bogomolov bound 31

5.2 Matter spectra in heterotic theories 32

5.3 The spectral cover construction 34

5.3.1 Spectral covers 34

$\begin{array}{lll}\text { 5.3.2 } & \text { Localized matter and spectral covers } & 36\end{array}$

$\begin{array}{ll}\text { 5.3.3 Limitations of spectral covers } & 37\end{array}$ 
6 Equivalence of constraints $\quad 38$

$\begin{array}{lll}6.1 & \text { Effective condition on } \eta_{i} & 39\end{array}$

$\begin{array}{lll}6.2 & \text { Effective constraint and gauge groups } & 39\end{array}$

6.3 F-theory constraints and $\mathrm{SO}(32)$ models $\quad 40$

6.4 Base-point free condition 40

6.5 A note of caution: G-flux 42

7 Examples $\quad 43$

$7.1 \mathbb{P}^{1}$ bundles over $\mathbb{P}^{2}$

$7.2 \quad \mathbb{P}^{1}$ bundles over $\mathbb{F}_{m}$

7.3 An F-theory model over $B_{2}=\mathbb{F}_{3}$ with a codimension two singularity but no gauge group $\quad 46$

$\begin{array}{lll}7.4 & \mathrm{dP}_{2} & 46\end{array}$

$\begin{array}{lll}7.5 & \mathrm{dP}_{3} & 47\end{array}$

$\begin{array}{lll}7.6 & \mathrm{dP}_{4} & 48\end{array}$

7.7 An example of an upper and lower bound on $\eta \quad 48$

$\begin{array}{ll}7.8 & \text { Examples with non-trivial chiral matter }\end{array}$

7.9 Generic G-flux that breaks gauge symmetry 52

8 Consequences for heterotic bundles $\quad 53$

9 Enumeration of heterotic/F-theory dual pairs with toric bases $B_{2} \quad 57$

10 Conclusions and open questions $\quad 61$

10.1 Detailed physics of smooth heterotic/F-theory dual pairs 61

10.2 Expanding heterotic/F-theory duality 64

A Properties of elliptically fibered Calabi-Yau three- and fourfolds $\quad 65$

B A brief exploration of rigid bundles $\quad 66$

\section{Introduction and overview}

\section{$1.1 \quad$ Introduction}

Since the early days of string theory it has been known that a wide range of different physical theories in four and higher dimensions can be realized by compactifying ten-dimensional string theories (and their more recently discovered higher-dimensional relatives M-theory and F-theory) on different geometric spaces [1,2]. Tremendous effort has been expended in exploring the range of theories that can be realized through such compactification. While for theories in higher dimensions with extended supersymmetry, the range of possible string models has a tractable scope, for theories in four dimensions with minimal supersymmetry known constructions seem to give rise to such a vast "landscape" [3-5] of possibilities that 
it is difficult to systematically study the set of allowed models and the constraints that they impose on $4 \mathrm{D}$ physics. It is suspected, in fact, that the known constructions of $4 \mathrm{D}$ $\mathcal{N}=1$ theories from string theory may represent only the tip of a much larger iceberg composed of compactifications described by more general mathematical objects including non-Kähler and non-geometric compactifications.

Nonetheless, it may be possible by analyzing specific string constructions to ascertain some global constraints and systematic features of the theories that arise from compactification of string theory. Recent work on globally classifying 6D string/F-theory compactifications and associated constraints on $6 \mathrm{D}$ supergravity theories [6-8] suggests that a systematic analysis is possible in six dimensions and may provide tools for a similar treatment of some aspects of the space of $4 \mathrm{D}$ compactifications [9]. In this paper we analyze how geometric constraints on two general classes of string compactifications to $4 \mathrm{D}$ are related, as a step towards a more systematic understanding of the space of $4 \mathrm{D} \mathcal{N}=1$ theories that can arise from string theory.

Compactifications of heterotic string theory and F-theory provide two corners of the string landscape where 4-dimensional $\mathcal{N}=1$ supersymmetric theories with chiral matter and exceptional gauge symmetries arise naturally. There is a tremendous literature on heterotic string compactifications; some recent work has sought to explore and enumerate the possible effective theories that can be obtained from compactification of the heterotic theory on a smooth Calabi-Yau (see [10-18] for some recent systematic studies). The duality between smooth heterotic compactifications and equivalent 4D F-theory constructions has also been broadly explored; see e.g. [19, 20]. The effective low-dimensional theories arising from compactifications of both heterotic string theory and F-theory are highly constrained by the background geometry of the compact dimensions. Indeed, it is an attractive possibility that these constraints might be strong enough to characterize which effective theories can arise (in any dimension) from heterotic or F-theory compactifications, or in the case of compactifications to 4 dimensions, used to characterize which string geometries could be relevant for string phenomenology and give rise to the low-energy physics we see in nature.

A major obstacle in any systematic attempt to classify the possible compactification geometries and effective theories for either the heterotic string or F-theory is the current limitation on our mathematical understanding of the relevant geometries. It is not known, for example, whether the number of distinct diffeomorphism classes of Calabi-Yau threefolds and fourfolds is even finite, much less how to characterize all the properties of the manifolds that determine the effective theories. There is, however, at least one class of backgrounds, involving dual heterotic and F-theory compactifications on elliptically fibered Calabi-Yau threefolds and fourfolds, where the number of topologically distinct string geometries is finite, and some systematic analysis is possible.

For those theories that have dual heterotic and F-theory constructions, the compactification geometries take the form $[21,22]$

$$
\text { Heterotic on } X_{n}, \quad \pi_{h}: X_{n} \stackrel{\mathbb{E}}{\longrightarrow} B_{n-1} \Leftrightarrow \text { F-theory on } Y_{n+1}, \quad \pi_{f}: Y_{n+1} \stackrel{K 3}{\longrightarrow} B_{n-1}
$$

where $X_{n}$ is elliptically fibered over $B_{n-1}$ and the K3-fibered manifold $Y_{n+1}$ admits a more detailed description as an elliptically-fibered Calabi-Yau $(n+1)$-fold with section over a 
base $\mathcal{B}_{n}$ which is itself $\mathbb{P}^{1}$ fibered over $B_{n-1}$. The classification of such dual theories can be done at increasing levels of complexity by including successively more information about the geometry. At the coarsest level, the theories can be classified by the topological type of the base $B_{n-1}$. In dimensions eight and six there is a unique $B_{n-1}$ associated with smooth heterotic/F-theory dual pairs (respectively a point and $\mathbb{P}^{1}$ ), but in four dimensions there are many distinct possible bases $B_{n-1}$. At the next level of detail, theories can be classified by the geometry of the F-theory base $\mathcal{B}_{n}$. For any given $B_{n-1}$ there are in general many distinct topological types of $\mathcal{B}_{n}$ that characterize allowed F-theory geometries. Theories with different bases $\mathcal{B}_{n}$ (including those with different $B_{n-1}$ ) are connected on the Ftheory side by tensionless string transitions and on the heterotic side by small instanton transitions [22-24]. We primarily focus in this paper on classifying theories at the level of $\mathcal{B}_{n}$. The choice of $\mathcal{B}_{n}$ on the F-theory side fixes some of the topology of the dual heterotic bundles, but not all (specifically, it fixes some components of the second Chern classes of the bundles). For a given choice of $\mathcal{B}_{n}$, Higgsing/unHiggsing transitions in the effective theory, which correspond to deforming along/tuning moduli in the F-theory and heterotic bundle pictures, can modify the gauge group of the low-energy effective theory, and correspondingly modify the bundle structure group on the heterotic side. For $\mathcal{N}=1$ theories in $4 \mathrm{D}, G$-flux on the F-theory side lifts some moduli and can give disjoint sets of string vacua associated with compactifications on a given $\mathcal{B}_{n}$. For the most part, in this paper we concentrate on features that depend only on the geometry of $\mathcal{B}_{n}$ and are independent of the moduli lifting and other issues associated with $G$-flux. While a further understanding of the consequences of $G$-flux is clearly desirable, a good understanding of the underlying geometric structure that we focus on in this paper seems to be an important first step in a systematic understanding of general 4D F-theory models.

In this work we focus on 4-dimensional effective theories arising from heterotic string theory on a smooth elliptically fibered Calabi-Yau threefold and F-theory on a dual K3fibered Calabi-Yau fourfold that admits a compatible elliptic fibration and has a smooth resolution. For both the Calabi-Yau threefold and fourfold geometries, we consider only geometries where the elliptic fibrations admit a (single) section. We assume that the gauge bundle in the heterotic theory is smooth, and that there are no heterotic 5-branes wrapping curves in the base $B_{2}$, which would be associated with singular small instanton configurations. $^{1}$

Beginning on the heterotic side of the duality, it is known that the number of topological types of smooth elliptically fibered Calabi-Yau threefolds with section is finite [25] (see also [7], for a more constructive argument in the context of Weierstrass models). In a heterotic dimensional reduction, the 10-dimensional gauge field and the vacuum gauge field configuration over the Calabi-Yau threefold must be taken into account. These are described in the $E_{8} \times E_{8}$ heterotic theory by adding to the Calabi-Yau geometry a pair of holomorphic vector bundles $V_{i}(i=1,2)$ on $X_{3}$ with structure groups $H_{i} \subseteq E_{8}$. In the $\mathrm{SO}(32)$ heterotic theory, only a single vector bundle is used. For fixed bundle topology

\footnotetext{
${ }^{1}$ In some cases, the dual geometries we consider may include heterotic 5-branes wrapping the elliptic fiber of the CY threefold. See [19] for a discussion of the different roles that heterotic 5-branes can play.
} 
(specified by $\operatorname{rank}(V)$ and a total Chern class, $c(V)$ ), it is known that the moduli space of bundles ${ }^{2}$ compatible with $\mathcal{N}=1$ supersymmetry in $4 \mathrm{D}$ has only finitely many components $^{3}[26,27]$. Although these proofs of general finiteness are at present not constructive, it seems possible to systematically construct at least one important class of dual models.

As we discuss further in section 3 , when the geometries are smooth on both the heterotic and F-theory sides the base surface $B_{2}$ is restricted to be a generalized del Pezzo surface [28], of which there are a finite number of topologically distinct types. Over these bases a rational $\left(\mathbb{P}^{1}\right)$ fibered threefold $\mathcal{B}_{3}$ must be built and here we restrict our attention to the case where $\mathcal{B}_{3}$ can be constructed as a $\mathbb{P}^{1}$ bundle over $\mathcal{B}_{2}$. We demonstrate here that there are a finite number of topologically distinct $\mathbb{P}^{1}$ bundles over any generalized del Pezzo surface such that $\mathcal{B}_{3}$ supports an elliptically fibered Calabi-Yau fourfold. Moreover, we show that this finite set of $\mathcal{B}_{3}$ 's can be enumerated for any $B_{2}$. The number of distinct branches of the moduli space of Weierstrass models over any specific $\mathcal{B}_{3}$ corresponding to distinct gauge group and matter contents is finite by a similar argument to that for base surfaces in (section 6.5 of) [7].

This class of dual heterotic/F-theory models thus represents a reasonable starting point with which we can get a first foothold into the problem of classifying and characterizing $4 \mathrm{D} \mathcal{N}=1$ string vacua and their effective theories, as well as understanding constraints on the effective theories arising from string geometry. ${ }^{4}$

The general structure just detailed is illustrated clearly in the simple case of $4 \mathrm{D}$ models where the base $B_{2}$ is toric. The powerful mathematical toolkit of toric geometry allows for simple and direct computations in this class of examples. While there are hundreds of generalized del Pezzo surfaces $B_{2}$ that can act as bases of smooth dual heterotic/F-theory Calabi-Yau threefolds and fourfolds, only 16 of these $B_{2}$ 's are toric. The direct enumeration of all associated F-theory bases $\mathcal{B}_{3}$ (built as $\mathbb{P}^{1}$ bundles) is a straightforward calculation, which we carry out in this paper as an example of the general theoretical framework.

\subsection{Overview of main results}

For the convenience of the reader, we summarize here some of the main results of the paper that we believe have some novelty, and indicate where in the paper these results are described in more detail.

\footnotetext{
${ }^{2}$ More precisely, the moduli space of Mumford semi-stable sheaves on $X_{3}$.

${ }^{3}$ Finiteness of the number of heterotic geometries here is established in two steps. First, the results of $[26,27]$ guarantee that for stable, hermitian bundles with fixed first and second Chern classes $c_{1}, c_{2}$ there are a finite number of possible values for the third Chern class $c_{3}(V)$ (note that in the case exceptional structure groups, $c_{3}$ is no longer a topological invariant). To argue that the number of heterotic geometries is finite we must further observe that $c_{1} \equiv 0 \bmod 2$, and the second Chern class is bounded as $0 \leq c_{2}(V) \leq$ $c_{2}\left(T X_{3}\right)$ by heterotic anomaly cancelation (see eq. (5.2)).

${ }^{4}$ As this paper was being completed, the paper [29] appeared, in which magnetized brane models were considered over smooth elliptically fibered Calabi-Yau threefolds over del Pezzo bases, and the number of models in this class was shown to be finite.
} 


\subsubsection{Classification and enumeration of models}

\section{Classification of $\mathbb{P}^{1}$-bundle bases $\mathcal{B}_{3}$ for $\mathbf{F}$-theory models with smooth heterotic}

duals

As described at the conclusion of the previous section, we show in section 4.1 that there are a finite number of $\mathbb{P}^{1}$ bundles $\mathcal{B}_{3}$ over smooth bases $B_{2}$ for $F$-theory models with smooth heterotic duals on elliptically fibered Calabi-Yau threefolds with section. This follows from the fact that the number of generalized del Pezzo surfaces $B_{2}$ is finite, and the number of possible "twists" of the $\mathbb{P}^{1}$ bundle $\mathcal{B}_{3}$ over any $B_{2}$ is finite. We construct explicit bounds on the twist that reduce the classification of $\mathcal{B}_{3}$ 's to a finite enumeration problem in section 4.1.3, and write a simple set of topological conditions that characterize allowed $\mathcal{B}_{3}$ 's in section 4.1.5 and section 4.1.6. These results are quite general, and do not depend on toric geometry or any other specific conditions on the F-theory base geometry beyond the $\mathbb{P}^{1}$ bundle structure.

\section{Enumeration of models with toric $B_{2}$ and smooth heterotic duals}

As a concrete example of the general classification results, we explicitly construct all $\mathrm{F}$ theory bases $\mathcal{B}_{3}$ that can be built as $\mathbb{P}^{1}$-bundles over toric surfaces $B_{2}$ giving rise to smooth elliptically fibered fourfolds for F-theory compactifications with smooth heterotic duals. For the 16 toric $B_{2}$ 's we find 4962 threefolds $\mathcal{B}_{3}$, and classify the generic associated effective theories. These manifolds add to the dataset of Calabi-Yau fourfolds that have been systematically studied to date (see [30-36]). These results are described in section 9 .

\subsubsection{Topological constraints on symmetries and spectra}

\section{Matching geometric F-theory constraints and heterotic bundle constraints}

We show that there is a close correspondence between the geometric constraints on Ftheory models and conditions for the existence of smooth, slope-stable bundles in heterotic theories. This extends earlier work of Rajesh [37] and Berglund and Mayr [38]. The details of this correspondence are elaborated in section 6 . Some of the most interesting aspects of this correspondence arise when a constraint is better understood on one side of the duality than the other. In particular, the next two items describe constraints on the gauge group and bundle structure that are currently understood most clearly from the F-theory perspective, while the last item below describes aspects of matter content that are clearest from the heterotic point of view.

\section{Heterotic bundles and the base-point free condition}

One of the most general methods known for explicitly constructing bundles suitable for heterotic compactification is the spectral cover construction [19, 39]. This construction is used to build bundles with structure group $\mathrm{SU}(N)$ or $\mathrm{Sp}(N)$. Irreducible bundles can only be constructed via a spectral cover when the second Chern class of the bundle satisfies a condition of base-point freedom. We find that for $\mathrm{SU}(N)$ or $\operatorname{Sp}(N)$ structure groups the base point freeness condition can be derived from the F-theory geometry independent of the assumption of any particular method of bundle construction. Thus, for these structure 
groups base-point-freeness of (a part) of the second Chern class is required for all bundles in the moduli space.

In addition, we find that many F-theory models that should have smooth heterotic duals violate this base-point free condition. We show that these are all associated with bundles having exceptional or $\mathrm{SO}(8)$ structure groups and thus do not violate the above constraints for $\mathrm{SU}(N)$ and $\operatorname{Sp}(N)$ bundles. More general methods such as the cameral cover construction [40-42] and other approaches to constructing general $G$-bundles described in [19] based on a theorem of Looijenga [43, 44] can in principle provide constructions of bundles with these more general structure groups. Our analysis gives a general classification of situations in which bundles with exceptional structure groups are expected to exist - though in many cases explicitly describing the properties of such bundles is an open problem in geometry. The property of base-point-freedom and its violation also has important consequences for the problem of vector bundle deformations and symmetry group breaking/enhancement. The base-point free condition is described in section 5.3. The corresponding F-theory condition and circumstances for its failure are described in section 6.4. Examples of cases where this condition is violated in F-theory are described in sections 7,9 .

\section{Limitations on gauge enhancement}

Geometric constraints on the F-theory side not only provide a minimal gauge group for the low-energy theory given a compactification topology, but can also limit the extent to which the gauge group can be enhanced over a given base geometry. For example, in many situations $\mathrm{SU}(2)$ and $\mathrm{SU}(3)$ gauge groups are constrained by F-theory geometry so that they cannot be in a broken phase of an $\mathrm{SU}(5)$ gauge group. In these cases the restriction is associated with the structure of a codimension one singularity in the F-theory geometry. In other cases, codimension two singularities related to matter fields constrain enhancement - so that, for example, in some cases an $E_{6}$ cannot be enhanced to an $E_{7}$. These limitations on gauge enhancement are described in section 4.2 and section 8 with examples given in section 7 .

\section{Chiral matter}

The circumstances under which the low-energy theory has chiral matter are better understood on the heterotic side. We identify a class of situations in which chiral matter must arise due to the heterotic geometry, with implications for the dual F-theory model when G-flux is incorporated. On the heterotic side these correspond to bundles built via the spectral cover construction with structure group given by $\mathrm{SU}(2 n+1)$, giving rise to $4 \mathrm{D}$ GUT theories with, for example, $E_{6}$ or SU(5) symmetry [45]. In particular, in the dual F-theory geometries enumerated in this work, we find that many examples of theories with generic $E_{6}$ symmetry contain chiral matter. Examples of this type are described in section 7.8.

\subsection{Outline}

This paper is organized as follows: we begin in section 2 with a brief review of the duality between heterotic string theory and F-theory in dimensions eight and six. We focus on the nature of heterotic/F-theory duality, the classification of models, and constraints 
on the effective theory, illustrating features and tools that are helpful in analyzing fourdimensional compactifications in the remainder of the paper. In section 3 we summarize heterotic/F-theory duality in four dimensions and describe the range of constructions of interest. Section 4 gives a more detailed description of the geometric constraints on the F-theory side, and section 5 describes the constraints on both the Calabi-Yau threefold and bundle geometries on the heterotic side. In section 6 we compare the constraints on the two sides and show when they are equivalent and when one side of the duality provides new information about the geometry of the other side. Section 7 contains some examples. In section 8 we summarize the consequences of our study for heterotic bundle moduli spaces, and in section 9 we describe the results of the systematic enumeration of all smooth Ftheory geometries with toric base $B_{2}$ and a smooth heterotic dual construction. Finally, a brief summary of this work and associated open questions are given in section 10. Some technical details are relegated to appendices.

\section{Lessons from heterotic/F-theory duality in higher dimensions}

\subsection{Heterotic/F-theory duality in eight dimensions}

Beginning with the initial formulation of F-theory in 8 dimensions [21, 46] (see [47] for a review), the duality of F-theory with the heterotic string has provided an important window through which both theories can be better understood. In 8 dimensions, F-theory compactified on an elliptically fibered $K 3$ surface $Y_{2}, \pi: Y_{2} \rightarrow \mathbb{P}^{1}$, is dual in certain (separate) limits of its parameter space to the perturbative $E_{8} \times E_{8}$ and $\mathrm{SO}(32)$ heterotic string theories on $T^{2}$. In the case of the $8 \mathrm{D} E_{8} \times E_{8}$ heterotic theory, this duality can be understood most explicitly in the weak coupling limit of the effective theory, which is realized by taking the volume of $T^{2}$ to be large in the heterotic theory. The heterotic $T^{2}$ volume modulus is mapped into a complex structure modulus of the $K 3$ surface in F-theory. Geometrically, the $E_{8} \times E_{8}$ limit corresponds to decomposing the $K 3$ surface into a (singular) fiber product of two elliptically fibered $d P_{9}$ surfaces, glued together along an elliptic curve ${ }^{5}$ - the so-called "stable degeneration limit" [19, 21, 22].

In the $8 \mathrm{D}$ stable degeneration limit, all the features of the two theories, including the moduli parameterizing the vacua, can be matched exactly [22, 46, 48-51]. For example, the possible gauge groups arising from different configurations of the heterotic flat gauge bundles on $T^{2}$ (i.e. Wilson lines) can be matched to the symmetries arising from ADE degenerations of the elliptic fiber of $K 3$ that produce different non-Abelian symmetries over points in the $\mathbb{P}^{1}$ base (corresponding to the positions of 7-branes in the language of Type IIB); these degenerations were classified mathematically by Kodaira [52, 53]. From the point of view of the classification of models and constraints, the $8 \mathrm{D}$ story is quite simple. In this case the base manifold $B_{0}$ is a point, and the F-theory base $\mathcal{B}_{1}=\mathbb{P}^{1}$ is the unique $\mathbb{P}^{1}$ bundle over this point. Thus, there is a single moduli space of $8 \mathrm{D}$ models connected by "Higgsing" type transitions that reduce or increase the size of the gauge group by de-tuning

\footnotetext{
${ }^{5}$ For the limit which produces the $\mathrm{SO}(32)$ heterotic theory, the $K 3$ degenerates into a fiber-product of rational surfaces, see [48] for details.
} 
or tuning moduli to modify the singularity structure of the elliptic fibration. On both the F-theory and heterotic sides, the only constraint is that the gauge algebra $\mathcal{G}$ must have a root lattice that can be embedded into the unique signature $(2,18)$ unimodular lattice $\Gamma^{2,18}([54]$, reviewed in [55]).

In lower dimensions, heterotic/F-theory duality is understood by fibering the $8 \mathrm{D} \mathrm{du}-$ ality over a nontrivial shared base manifold $B_{n-1}$. As in (1.1), a heterotic theory on an elliptically fibered Calabi-Yau $n$-fold $X_{n}, \pi_{h}: X_{n} \rightarrow B_{n-1}$ is dual to F-theory on a $K 3$ fibered $(n+1)$-fold $Y_{n+1}$ with the same base, $\pi_{f}: Y_{n+1} \rightarrow B_{n-1}$, in which the $K 3$ fiber is in turn elliptically fibered as described above. The elliptic and $K 3$ fibrations are taken to be compatible, and both are chosen to have sections. This duality has been studied primarily in the stable degeneration limit [19], though in this paper we describe aspects of the duality that are true more generally, independent of this limit.

\section{$2.2 \quad$ Heterotic/F-theory duality in six dimensions}

\subsubsection{Dual 6D geometries}

For dual heterotic/F-theory compactifications to six dimensions, the perturbative heterotic compactification space is a K3 surface that is elliptically fibered over the common base $B_{1}=\mathbb{P}^{1}$, and the dual F-theory geometry is a Calabi-Yau threefold $Y_{3}$ that is elliptically fibered with section over a Hirzebruch surface, $\pi: Y_{3} \rightarrow \mathbb{F}_{n}$, where the Hirzebruch surface $\mathcal{B}_{2}=\mathbb{F}_{n}$ is itself a $\mathbb{P}^{1}$ bundle over $B_{1}$. As in $8 \mathrm{D}$, codimension one singularities in the elliptic fibration encode a gauge group in the F-theory picture, which in $6 \mathrm{D}$ can include non-simply laced groups when monodromy is present [56]. Codimension two singularities encode matter fields.

In principle, heterotic/F-theory duality can be extended beyond the set of smooth dual geometries by incorporating non-perturbative effects such as NS5-branes in the heterotic theory. In this case, the dual F-theory geometry $Y_{3}$ is an elliptic fibration over a more general 2-dimensional base $\mathcal{B}_{2}$, which is a blow-up of a Hirzebruch surface [21, 22], corresponding to a more general $\mathbb{P}^{1}$ fibration over $B_{1}=\mathbb{P}^{1}$. The base $\mathcal{B}_{2}=\mathbb{P}^{2}$ can also be realized on the F-theory side, e.g. after a tensionless string transition from $\mathcal{B}_{2}=\mathbb{F}_{1}[21,24]$. For each choice of $\mathcal{B}_{2}$, there is a connected moduli space of elliptically fibered Calabi-Yau threefolds describing a set of $6 \mathrm{D}$ theories connected by Higgsing and unHiggsing transitions. The global space of 6D F-theory compactifications [7] consists of a finite family of such moduli spaces connected by tensionless string type transitions. The connectivity of the set of moduli spaces associated with distinct $\mathcal{B}_{2}$ 's corresponds to the mathematical framework of minimal surface theory [57, 58], in which curves of self-intersection -1 are blown down until a minimal surface (in this case a Hirzebruch surface $\mathbb{F}_{n}$ with $n \leq 12$, $\mathbb{P}^{2}$, or the Enriques surface [59]) is reached. A systematic classification of F-theory bases $\mathcal{B}_{2}$ according to the intersection properties of effective divisors is given in [8]. A complete enumeration of allowed bases is in principle possible and has been carried out explicitly for toric $\mathcal{B}_{2}$ 's [60], and the more general "semi-toric" class of $B_{2}$ 's that admit a single $\mathbb{C}^{*}$ action [61]. The global description of the moduli space is much more complicated on the heterotic side, where multiple coincident small instantons must be analyzed systemati- 
cally (section 2.2.4). We restrict attention in this paper to smooth heterotic/F-theory dual geometries where no small instantons arise, which in $6 \mathrm{D}$ limits us to $\mathcal{B}_{2}=\mathbb{F}_{n}$.

\subsubsection{Geometric conditions on vacua}

Even in $6 D$, each side of the duality encodes some nontrivial information about the geometry of its dual theory. In early explorations of F-theory [21, 22, 56], the degrees of freedom and effective theory of heterotic compactifications were used to develop the "dictionary" of how the dual Calabi-Yau threefold geometry determines the gauge symmetries and matter spectra in the F-theory description. F-theory can in turn be used to enumerate possible heterotic backgrounds $\left(K 3, V_{1}, V_{2}\right)$ and to make useful statements about their properties.

One of the most significant aspects of this duality is the way that the dual theories realize the condition for $\mathcal{N}=1$ (minimal) supersymmetry in 6 dimensions. On the $\mathrm{F}$ theory side this appears as the condition that the total elliptically fibered compactification space is a Calabi-Yau threefold (more precisely, the manifold can be singular, in which case the resolved geometry is a Calabi-Yau threefold; the F-theory description can be thought of as a singular M-theory limit, as reviewed for example in [47]). On the heterotic side, this condition corresponds to the statement that the compactification manifold is a $K 3$ surface and that the gauge bundles $\left(V_{1}, V_{2}\right)$ with structure groups embedded into each $E_{8}$ factor satisfy the Hermitian Yang-Mills equations [1], that is, that they are slope(poly)stable [62-64]. Furthermore, the first Chern class of the principal bundles must vanish, ${ }^{6}$ with $c_{1}\left(V_{i}\right) \equiv 0(\bmod 2)$.

The choice of a smooth heterotic/F-theory dual pair in 6D is determined by a single integer. In the heterotic theory this appears as the choice of a fixed second Chern class for the vector bundles $c_{2}\left(V_{1,2}\right)=12 \pm n$. On the F-theory side, this corresponds to the choice of Hirzebruch surface $\mathbb{F}_{n}$ for the two-fold base $\mathcal{B}_{2}$. The constraint $n \leq 12$ (originally described in [21]) gives a simple example of the type of geometric constraint that we explore later in this paper for $4 \mathrm{D}$ compactifications. On the heterotic side this constraint follows from the slope-stability of the vector bundles and the heterotic anomaly cancellation condition which relates the second Chern class of the holomorphic tangent bundle of K3 with those of the gauge bundles $V_{1,2}$. On the F-theory side this constraint follows from the fact that for $n>$ 12 the existence of an effective divisor in $\mathcal{B}_{2}=\mathbb{F}_{n}$ with self-intersection $-n<-12$ yields a singularity in any elliptic fibration over $\mathcal{B}_{2}$ that cannot be resolved to yield a total space that is a Calabi-Yau manifold. Thus, in this case rather different geometric considerations on the two sides give the same analytic constraint on the structure of the allowed theories.

\subsubsection{Moduli and the stable degeneration limit}

It is worth briefly reviewing the moduli of heterotic/F-theory compactifications in $6 D$ and the interpretation of these moduli in the two dual pictures. As described in $[21,22]$ and

\footnotetext{
${ }^{6}$ More precisely, since the generators of $E_{8}$ are traceless, all principal bundles $\mathcal{V}$ that are sub-bundles of an $E_{8}$ bundle (i.e. that have structure group $H \subseteq E_{8}$ ) must have vanishing first Chern class. However, if the associated vector bundles $V, \wedge^{2} V \ldots$ (arising in the heterotic theory from the decomposition of the 248-dimensional adjoint of $\left.E_{8}\right)$ are reducible, then their first Chern classes can be non-zero and satisfy $c_{1}\left(V_{i}\right) \equiv 0(\bmod 2)$.
} 
easily confirmed in the toric description (reviewed in section 3.3), a Calabi-Yau threefold $Y_{3}$ that is elliptically fibered over the Hirzebruch surface $\mathbb{F}_{n}$ can be described in Weierstrass form as

$$
y^{2}=x^{3}+f\left(z_{1}, z_{2}\right) x+g\left(z_{1}, z_{2}\right)
$$

where $\left(z_{1}, z_{2}\right)$ are coordinates on $\mathbb{F}_{n}$ and

$$
\begin{array}{ll}
f\left(z_{1}, z_{2}\right)=\sum_{i=0}^{I} z_{1}^{i} f_{8+n(4-i)}\left(z_{2}\right) & I \leq 8 \text { such that } 8+n(4-I) \geq 0 \\
g\left(z_{1}, z_{2}\right)=\sum_{j=0}^{J} z_{1}^{j} g_{12+n(6-j)}\left(z_{2}\right) & J \leq 12 \text { such that } 12+n(6-J) \geq 0
\end{array}
$$

More abstractly, $f, g$ are sections of the line bundles $\mathcal{O}(-4 K), \mathcal{O}(-6 K)$, where $K$ is the canonical class of the base $\mathcal{B}_{2}$; these explicit expressions give a local coordinate description of generic sections of these line bundles.

As is now well understood $[19,21,22]$, the heterotic/F-theory dictionary in $6 \mathrm{D}$ indicates that the "middle" polynomials (the coefficients of $z_{1}^{4}$ and $z_{1}^{6}$ in $f$ and $g$, respectively in $(2.2),(2.3))$ correspond to the moduli of the heterotic $K 3$ surface, while polynomials of low degree (coefficients of $z_{1}^{i}$ with $i<4$ in $f$ and coefficients of $z_{1}^{j}$ with $j<6$ in $g$ ) parameterize one of the heterotic bundles $V_{1}$, and polynomials of high degree $(i>4, j>6)$ parameterize the other bundle $V_{2}$. The bundle $V_{1}$ has structure group $H_{1}$, which is embedded in $E_{8}$, and the resulting gauge group is the commutant $G_{1}$ of $H_{1} \subseteq E_{8}$. On the F-theory side this corresponds to a Calabi-Yau threefold with 7-branes wrapping the $\mathbb{P}^{1}$ base of the $K 3$-fibration, giving rise to symmetry $G_{1}$ encoded in the singularity structure of the elliptic fibration at the point $z_{1}=0$. Similarly, the second heterotic bundle $V_{2}$ has structure group $\mathrm{H}_{2}$ with commutant $G_{2}$, associated with the singularity structure of the F-theory elliptic fibration at $z_{1}=\infty$. This correspondence can be made precise in the stable degeneration limit, in which $Y_{3} \rightarrow Y_{1} \cup_{K 3} Y_{2}$ where $Y_{1,2}$ are (non-CY) $d P_{9}$-fibered threefolds. In this limit, the infinitesimal deformation space, $\operatorname{Def}\left(Y_{i}\right)$, of $Y_{i}$ can be matched exactly to that of the bundles $\operatorname{Def}\left(V_{i}\right)$ and the $K 3$ surface, $\operatorname{Def}(K 3)$ (see $[21,22,56,65]$ and [66] for a modern treatment of this result in terms of limiting mixed Hodge structures). That is,

$$
h^{2,1}\left(Y_{i}\right)=h^{1}\left(K 3, \operatorname{End}_{0}\left(V_{i}\right)\right)+20
$$

The correspondence between the F-theory moduli in the Weierstrass model and moduli of the dual heterotic bundles is particularly transparent in the spectral cover construction (5.3), where the polynomials $f_{k}, g_{k}$ play a dual role in parameterizing the spectral cover divisor on the heterotic side.

\subsubsection{Constraints on bundles and gauge symmetry}

For a fixed topology of $\mathcal{B}_{2}$ on the F-theory side, the moduli encoded in the functions $f, g(2.2),(2.3)$ parameterize Weierstrass models for all elliptically (and $K 3$ ) fibered threefolds $Y_{3}$ over the base $\mathcal{B}_{2}$. Parts of this moduli space in principle give a complete encoding of each dual heterotic moduli space of sheaves with fixed total Chern class, denoted 
$\mathcal{M}_{\omega}\left(\mathrm{rank}, c_{1}, c_{2}\right)$, that are stable with respect to a chosen Kähler form ${ }^{7} \omega$ on $K 3$. In this context, nontrivial features of the heterotic and F-theory geometries can be exactly matched. Considering only F-theory on the elliptically fibered threefolds with the 13 Hirzebruch bases $\mathbb{F}_{n}, n=0, \ldots 12$, it is possible to deduce a number of facts about the dual moduli spaces of sheaves $\mathcal{M}_{\omega}\left(\mathrm{rank}, c_{1}, c_{2}\right)$. The first of these is that for $c_{1}=0$, fixed $c_{2}$, and the structure group $H_{i}$ of $V_{i}$ fixed subject to $H_{i} \subseteq E_{8}, \mathcal{M}_{\omega}$ has only one component - corresponding to a connected deformation space of a dual Calabi-Yau threefold $Y_{3}$ as described above. ${ }^{8}$

The moduli space structure on the F-theory side is matched non-trivially not only to the irreducibility of $\mathcal{M}_{\omega}\left(\mathrm{rank}, c_{1}, c_{2}\right)$ for fixed rank, but also provides information about deformations of $V_{i}$ that change the rank. A change in the rank (and hence structure group $H_{i}$ ) of the bundles $V_{i}$ corresponds to changing the gauge symmetry of the $6 D$ effective theory $\left(G_{i} \subseteq E_{8}\right)$. In the F-theory geometry, this gauge symmetry can be varied by changing the complex structure of $Y_{3}$. By tuning the complex structure to special loci in moduli space, it is possible to augment the Kodaira singularity types of the elliptic fibration over divisors in the base, which enhances the gauge group symmetry of the $6 \mathrm{D}$ theory; this corresponds on the heterotic side to specializing $V_{i}$ to bundles with smaller structure groups. In the reverse process, in cases where complex structure deformations exist to break a symmetry, there is a simple realization in the effective theory as a direct "Higgsing" of $G$ by charged hypermultiplets. Complete "chains" of these breaking/enhancement patterns have been determined for the dual $6 D$ theories and matched exactly to complex structure deformations of the corresponding threefolds $Y_{3}$ [56] (see also figure 1).

While the gauge symmetry of a $6 \mathrm{D}$ theory can often be made smaller or larger by Higgsing or un-Higgsing through de-tuning and tuning moduli in the F-theory picture, there are also constraints on both sides that can restrict the extent to which a gauge group can be broken or expanded. For $n \geq 3$, the threefolds $\pi: Y_{3} \rightarrow \mathbb{F}_{n}$ are generically singular (though they admit a smooth resolution). This means that in these cases there is a 6-dimensional gauge symmetry that cannot be Higgsed away by giving vevs to the hypermultiplets associated to the complex structure moduli. In the F-theory geometry, this corresponds to the presence of a divisor of self-intersection $-n$ in $\mathbb{F}_{n}$, over which the elliptic fibration must become singular and has a Kodaira type associated with a nontrivial gauge group factor. On the heterotic side, this implies that for certain values of the second Chern class $c_{2}$ there is a maximum structure group $H$ possible for any bundle with that topology, since $H \subseteq E_{8}$ encodes the unbroken symmetry $G$ in the 6-dimensional heterotic

\footnotetext{
${ }^{7}$ It is important to note that while heterotic/F-theory duality is believed to hold for the full moduli space of the the two theories, the explicit "dictionary" between degrees of freedom is only well understood for suitably weakly coupled regions of parameter space. On the heterotic side, this corresponds to moduli spaces of stable sheaves that are stable for the appropriate "adiabatic" choice [19] of Kähler form on $K 3$.

${ }^{8}$ To avoid confusion it should be noted here that the rank appearing in the definition of the moduli space $\mathcal{M}_{\omega}\left(\right.$ rank $\left., c_{1}, c_{2}\right)$ refers to a fixed fiber dimension for a given vector bundle appearing in a representation of $H_{i}$ determined by the decomposition of the adjoint of $E_{8}$, not the rank of $H_{i}$ as a Lie group. For example, although $F_{4}$ has rank 4 as a Lie group, one relevant moduli space for $F_{4}$ bundles appearing in heterotic theories would consist of rank 26 vector bundles. See section 5.2 for a general discussion of the relevant bundles, ranks and group representations.
} 


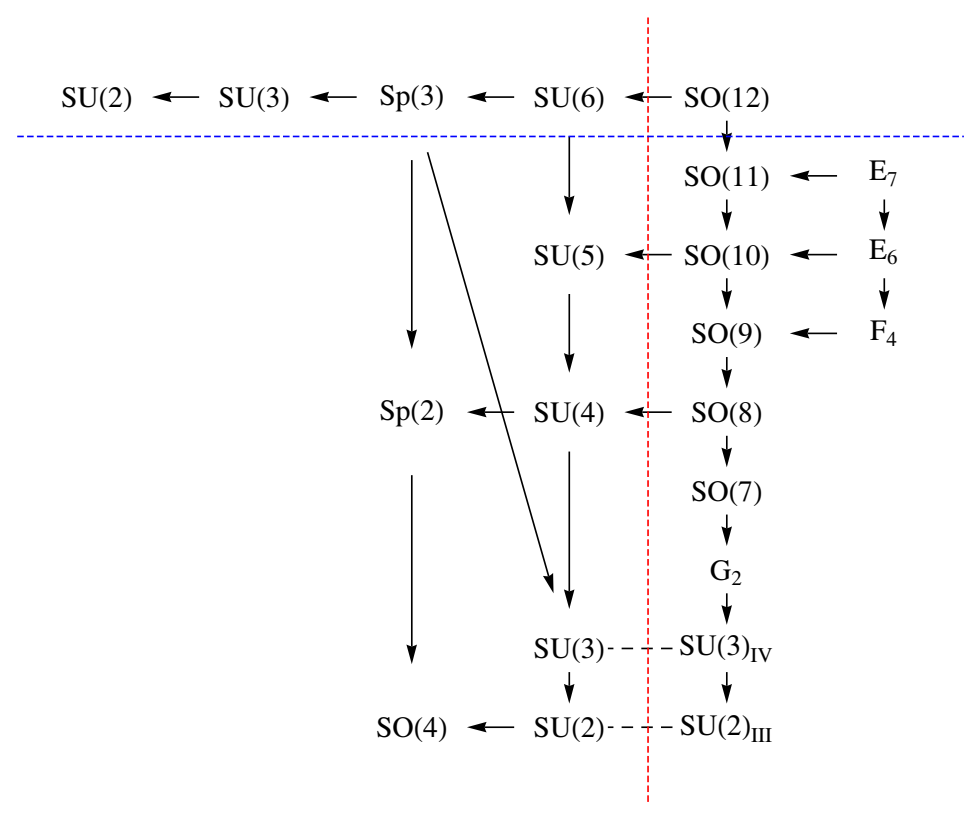

Figure 1. The possible Higgsing/Enhancement chains for smooth heterotic/F-theory dual pairs; modified from [56]. Figure depicts Higgsing possibilities based on heterotic bundles with structure group $H \subset E_{8}$, which match with dual F-theory models. F-theory gauge groups from Kodaira singularities with $f, g$ having nonzero degrees of vanishing lie to the right of the vertical red dashed line, such gauge groups can be forced from the geometry (geometrically "non-Higgsable") and cannot be unHiggsed to anything left of the line. The SU(3)'s and SU(2)'s connected near the bottom by horizontal dashed lines correspond to transitions between different Kodaira types in F-theory from type $I V, I I I$ to type $I_{3}, I_{2}$. The top row above the horizontal blue dashed line corresponds to an alternative Higgsing sequence from $E_{8}$ to $\mathrm{SU}(3), \mathrm{SU}(2)$ with non-standard commutants (e.g. $H=\mathrm{SU}(3) \times$ $G_{2}$ for upper $\mathrm{SU}(3)$ ), generically associated with matter in the adjoint representation, which on the F-theory side involves wrapping on higher genus curves for $6 \mathrm{D}$ models. Note that in F-theory models that do not have heterotic duals, further unHiggsing (e.g. to $\mathrm{SU}(N>6)$ ) can occur. Note also that in the heterotic theory some Higgsing chains lead to product gauge groups, as discussed further in text.

theory. For example, if $c_{2}(V)=4$, the maximal structure group of $V$ is $\mathrm{SU}(2)$, for any such bundle on $K 3$. It follows then that the moduli space of stable sheaves, $\mathcal{M}_{\omega}(r, 0,4)$ contains no locally free sheaves (i.e. smooth bundles) for $r>2$ (we will sometimes for brevity refer to such a moduli space as "empty"). This corresponds in F-theory to the fact that $\pi: Y_{3} \rightarrow \mathbb{F}_{8}$ is singular with a generic, non-Higgsable $E_{7}$ symmetry [22].

A fact that is perhaps not generally well appreciated is that there are also cases in $6 \mathrm{D}$ where there are nontrivial constraints on the ways in which a gauge group can be enhanced by "unHiggsing" the generic model in a given component of the moduli space (i.e. possible up/rightward paths towards $E_{7}$ or $\mathrm{SO}(12)$ in figure 1 ). In particular, in any $6 \mathrm{D}$ F-theory construction where the low-energy theory has a generic (non-Higgsable) gauge group SU(3) arising from a Kodaira type $I V$ singularity associated with a curve $C$ of self-intersection -3 , the group cannot be enhanced to any larger $\mathrm{SU}(N)$. In F-theory this follows from the fact that the Weierstrass coefficients $f, g$ must vanish to degrees 2,2 on $C$, which is 
incompatible with an $A_{n}$ type singularity for $n>2$. This condition corresponds on the heterotic side to a constraint on the extent to which the $E_{6}$ bundle over K3 with instanton number 9 can be deformed to a bundle with reduced structure group. In the case at hand, the enhancement $\mathrm{SU}(3) \rightarrow G_{2}$ (i.e., the reduction of the $E_{6}$ bundle $V_{27} \rightarrow V_{26} \oplus \mathcal{O}$ in $^{9} F_{4}$ ) is possible, while the enhancement $\mathrm{SU}(3) \rightarrow \mathrm{SU}(4)$ via $V_{27} \rightarrow V_{10}+V_{16} \oplus \mathcal{O}$ in $\mathrm{SO}(10)$ is not. Phrased differently, in terms of the Higgsing chains given in figure 1, if we begin with the lower right $\mathrm{SU}(3)_{I V}$ for this case, it is possible to move upwards (un-Higgsing) and to the right along the right-hand path $G_{2} \leftarrow \mathrm{SO}(7) \leftarrow \ldots$, but the group cannot be unHiggsed to the path $\mathrm{SU}(4) \leftarrow \mathrm{SU}(5) \leftarrow \ldots$. While in $6 \mathrm{D}$, those F-theory models with smooth heterotic duals have gauge groups that can always be enhanced to $E_{7}$ (or $\mathrm{SO}(12)$ ) along some path in figure 1, the obstacles to gauge group enhancement can be stronger for more complicated 6D F-theory models (without smooth heterotic duals), and in four dimensions there are a number of constraints of this type. We explore the $4 \mathrm{D}$ constraints to gauge group enhancement in more detail in section 4.2 .

The set of possible Higgsing/unHiggsing chains in dual pairs of 6D heterotic/F-theory models contains a number of other interesting features. As depicted in figure 1, a heterotic $E_{8}$ symmetry can be broken to $\mathrm{SU}(3)$ in several distinct ways, depending on whether the commutant is $H=E_{6}$ or $H=G_{2} \times \mathrm{SU}(3)$, for example. In the latter case, explicit computation of the branching rules generically gives matter in the adjoint representation for the smaller groups along the Higgsing chain. In 6D F-theory models, SU $(N)$ matter in the adjoint representation is only possible when the gauge group arises on a curve of higher genus in the F-theory base $B_{2}$. This is also true for $\mathrm{SU}(N)$ models containing any representation other than the fundamental and $k$-fold antisymmetric tensor representation [67-69]. For F-theory models on $\mathbb{F}_{m}$ dual to smooth heterotic models, the gauge group factors live on divisors of self-intersection $\pm m$. The divisors of self-intersection $-m$ with $m>0$ are rigid and cannot support a higher genus curve. The divisors of self-intersection $+m$, however, can be taken with higher multiplicity, giving a higher genus Riemann surface. For example, in $\mathbb{F}_{2}$ there are irreducible curves of genus one that have twice the divisor class of the irreducible curve $\tilde{S}$ of self-intersection +2 . An $\mathrm{SU}(3)$ gauge group with an adjoint results, corresponding to an $\mathrm{SU}(3)$ factor in the dual heterotic model with the non-standard commutant. Note that for $m \neq 0$ this can only happen on one side, so only one gauge group can have adjoint matter representations and lie on the top line of figure 1, corresponding on the heterotic side to the fact that only bundles with $c_{2}(V)>12$ can have a structure group such as $G_{2} \times \mathrm{SU}(3)$. Another interesting feature that can arise in this context is the appearance of product gauge groups as one of the factors $G_{i} \subset E_{8}$ in a heterotic model. On the F-theory side this corresponds again to a gauge group on a multiple of the divisor class with positive self-intersection, now given as a sum of two irreducible parts.

Constraints on heterotic bundles dual to F-theory models on $\mathcal{B}_{2}=\mathbb{F}_{n}$ are particularly strong for $n=9, \ldots 12$, in which cases the generic symmetry of $Y_{3}$ is $E_{8}$ (located on the patch containing $z_{1}=0$ in $\mathbb{F}_{n}$ ). This corresponds on the heterotic side to no structure group at all. That is, $H$ is trivial and full $E_{8}$ symmetry is unbroken. In these cases, no smooth

\footnotetext{
${ }^{9}$ Here $\mathcal{O}$ denotes the trivial line bundle over $X_{3}$.
} 
vector bundles exist on $K 3$ with $c_{2}=1,2,3$. On the F-theory side this corresponds to the fact that the $-9,-10$ or -11 curve in $\mathbb{F}_{m}$ must contain a point where the elliptic fibration is so singular that the point in the base must be blown up for the total space to have the structure of a Calabi-Yau threefold [8]. On the heterotic side this blowing up corresponds to the shrinking of an instanton to a point; in these cases there are sheaves (not locally free) with the desired topology. These cases go outside the smooth heterotic/F-theory dual paradigm that we focus on here; we encounter $4 \mathrm{D}$ analogues of these situations later but do not study them in detail. F-theory geometry and heterotic/F-theory duality thus lead to the inclusion of small instantons and sheafy degenerations in the heterotic picture, making it clear that the structure of the physical theory is in agreement with the mathematical notion of bundle/sheaf moduli spaces. Mathematically, any attempt to construct a moduli space of bundles alone results in a non-compact space. It is only by including sheaves (i.e., degenerations in the vector bundle) that a compact moduli space $\mathcal{M}_{\omega}$ arises. Physically, in the heterotic theory these sheaves ${ }^{10}$ correspond to point-like instantons (NS/M5 branes) on the K3 [21, $22]$, in the cases above with "instanton number" $c_{2}=1,2,3$. Thus for $0<c_{2}<4$ we note that the moduli space $\mathcal{M}_{\omega}$ is non-empty but contains only sheaves and no smooth bundles. In principle, this line of development could be used to develop a full description of the $6 \mathrm{D}$ moduli space of non-perturbative vacua from the heterotic side of the duality which could be matched to the geometric F-theory description, though we do not pursue this further here.

\subsubsection{Summary of $6 \mathrm{D}$ duality and relevance for $4 \mathrm{D}$}

To summarize this review of the $6 \mathrm{D}$ story, in 6 dimensions heterotic/F-theory duality encodes a deep and non-trivial correspondence between the moduli space of elliptically fibered Calabi-Yau threefolds (and their stable degeneration limits) and the moduli space of stable sheaves over $K 3$. In six dimensions, essentially all of the information that can be inferred from the F-theory geometry about the heterotic bundle moduli space, including a) the irreducibility of $\mathcal{M}_{\omega}\left(r, c_{1}, c_{2}\right)$ and b) the existence of a "maximal" rank/structure group for a given $c_{2}$, can be independently determined using known mathematics to study the heterotic geometry. Both of the facts, a) and b) were previously known in the mathematics literature in the study of moduli spaces of stable sheaves on $K 3$ and Donaldson-Thomas invariants on $K 3$ (see for example [72]).

In this paper, we ask many of the questions described above in the context of $4 \mathrm{D}$ heterotic/F-theory duality, which relates the moduli space of vector bundles $\left(V_{1}, V_{2}\right)$ on an elliptically fibered Calabi-Yau threefold to the moduli of a $K 3$ fibered Calabi-Yau four-fold. In this context, however, the information obtained is made more significant by the fact that far fewer mathematical techniques are known for determining the moduli space of stable sheaves on Calabi-Yau threefolds. Indeed, aside from a handful of examples with special topology (see e.g. [73-78]), no systematic tools exist for constructing the moduli spaces $\mathcal{M}_{\omega}\left(\mathrm{rank}, c_{1}, c_{2}, c_{3}\right)$ or the corresponding Donaldson-Thomas Invariants [79].

In the following sections we use heterotic/F-theory duality to develop analogous statements to those made above for bundles on $K 3$, and explore a number of new features,

\footnotetext{
${ }^{10}$ More precisely, skypscraper sheaves supported over points in the $\mathbb{P}^{1}$ base of $K 3$ [70, 71].
} 
unique to the 4D theory. These include deriving strong upper and lower bounds (based on $\left.c_{2}(V)\right)$ on the bundle structure group $H$, as well as constraints tying the matter spectrum of the theories to topology.

\section{Heterotic/F-theory duality in four dimensions}

We now briefly review the duality between heterotic string and F-theory compactifications for four-dimensional $\mathcal{N}=1$ supergravity theories [19, 80]. We begin with a general abstract formulation of the duality in section 3.1 , and then characterize the possible compactification geometries that are smooth on both sides of the duality in section 3.2. In section 3.3, we give a more detailed description of constructions that involve a toric base surface $B_{2}$.

\subsection{Geometry of heterotic/F-theory duality}

We focus on the best understood class of dualities, in which the heterotic compactification is on a smooth Calabi-Yau threefold $X_{3}$ that is elliptically fibered with a single section over a base $B_{2}$, the heterotic bundles are smooth and irreducible and there are no additional 5 -branes wrapping curves in the base. In the dual F-theory compactification, we consider a threefold $\mathcal{B}_{3}$ that is a $\mathbb{P}^{1}$ bundle over $B_{2}$. The F-theory compactification space $\mathcal{B}_{3}$ in turn acts as a base for an elliptically-fibered Calabi-Yau fourfold $Y_{4}$. Following [19], we can construct the $\mathbb{P}^{1}$ bundle $\mathcal{B}_{3}$ as a projectivization of a sum of two line bundles

$$
\mathcal{B}_{3}=\mathbb{P}(\mathcal{O} \oplus \mathcal{L}),
$$

where $\mathcal{L}$ is a general line bundle on the base $B_{2}$. On $\mathcal{B}_{3}$ we have the classes $R=$ $c_{1}(\mathcal{O}(1)), T=c_{1}(\mathcal{L})$, where $\mathcal{O}(1)$ is a bundle that restricts to the usual $\mathcal{O}(1)$ on each $\mathbb{P}^{1}$ fiber. There are sections $\Sigma_{-}$and $\Sigma_{+}=\Sigma_{-}+T$ of $\mathcal{B}_{3}$ that satisfy $\Sigma_{-} \cdot \Sigma_{+}=0$, corresponding to the relation $R(R+T)=0$ in cohomology.

An F-theory model on $\mathcal{B}_{3}$ is dual to a heterotic model on $X_{3}$. For the $E_{8} \times E_{8}$ heterotic theory, the bundle decomposes as $V_{1} \oplus V_{2}$, and the curvatures split as (see appendix A)

$$
\frac{1}{30} \operatorname{Tr} F_{i}^{2}=\eta_{i} \wedge \omega_{0}+\zeta_{i}, \quad i=1,2
$$

where $\eta_{i}, \zeta_{i}$ are (pullbacks of) 2-forms and 4 -forms on $B_{2}$ and $\omega_{0}$ is Poincaré dual to the section. The Bianchi identity gives $\eta_{1}+\eta_{2}=12 c_{1}\left(B_{2}\right)$. Heterotic/F-theory duality is possible when

$$
\eta_{1,2}=6 c_{1}\left(B_{2}\right) \pm T, \quad\left(E_{8} \times E_{8}\right) .
$$

This correspondence between $\eta_{i}$ and $T$ was identified by Friedman, Morgan, and Witten for bundles in the stable degeneration limit in [19]. It was shown more generally in [9] that this correspondence follows directly from the structure of axion-curvature squared terms in the dimensionally reduced supergravity action, independent of the stable degeneration limit or type of bundle construction. For the $\mathrm{SO}(32)$ heterotic string, the analysis [9] of the axion-curvature squared terms in the $4 \mathrm{D}$ supergravity action constrains the twisting $T$ of the bundle on the F-theory side to satisfy

$$
T=2 c_{1}\left(B_{2}\right), \quad(\mathrm{SO}(32))
$$


for a dual $\mathrm{SO}(32)$ heterotic compactification to exist. This generalizes the corresponding $6 \mathrm{D}$ case where the $\mathrm{SO}(32)$ heterotic theory is dual to F-theory on $\mathbb{F}_{4}(n=4 \Rightarrow T=4 H=$ $\left.2 c_{1}\left(B_{1}=\mathbb{P}^{1}\right)\right)$.

Note that we assume that the elliptic fibration on the heterotic side has precisely one section. It is possible that fibrations with more than one independent section (i.e., with nontrivial Mordell-Weil group) - or with multisections - may admit some more general kind of F-theory dual. We leave this interesting question for further work. On the F-theory side, elliptic fibrations without a global section were explored in [81], and included into the moduli space of Weierstrass models in [82]. As described in [82], such models can be understood from Higgsing abelian U(1) symmetries, which can in turn be understood from Higgsing nonabelian symmetries, all of which should have a clear parallel between the F-theory and heterotic descriptions, though we do not pursue this here.

For elliptic fibrations $X_{3}$ with one section and smooth total space, not all topological features of the heterotic bundle are determined by the dual F-theory geometry. Knowing the base $B_{2}$ and the twist $T$ of the dual F-theory $\mathbb{P}^{1}$ bundle (i.e., knowing $\mathcal{B}_{3}$ ) allows for the identification of all components of $c_{2}\left(V_{i}\right)$ except for $\zeta_{i}$ in (3.2); these components together satisfy $\zeta_{1}+\zeta_{2}=11 c_{1}\left(B_{2}\right)^{2}+c_{2}\left(B_{2}\right)$ (appendix A, [19]). The F-theory fourfold geometry also does not fix $c_{3}\left(V_{i}\right)$; for this we must consider in addition $G$-flux on $Y_{4}$. These features each correspond to one undetermined parameter on the heterotic side. It is interesting that many of the consequences that can be derived from F-theory for the structure of heterotic bundles - discussed later in this paper - are largely independent of any possible freedom in these two parameters on the heterotic side.

A central piece of information on which we focus in our analysis is the generic gauge group in the low-energy $4 \mathrm{D}$ supergravity theory corresponding to a given heterotic/F-theory dual pair. On the F-theory side, the threefold base $\mathcal{B}_{3}$ defined by $B_{2}$ and $T$ supports an elliptic fibration that may have singularities along certain divisors. The Kodaira classification of such singularities indicates the presence of nonabelian gauge group factors in the $4 \mathrm{D}$ supergravity theory $[21,22]$. There can only be a smooth heterotic dual when the only nonabelian gauge group factors are associated with the divisors $\Sigma_{-}, \Sigma_{+}$. For any given base $\mathcal{B}_{3}$, there is therefore a minimal gauge group $G=G_{1} \times G_{2}$, corresponding to singularity structures present over $\Sigma_{-}, \Sigma_{+}$in a completely generic elliptic fibration over $\mathcal{B}_{3}$. When such a gauge group is present, it implies that the largest possible structure group for a bundle on the heterotic side over $B_{2}$ with the topological data $\eta_{1,2}$ fixed by (3.3) is $H=H_{1} \times H_{2}$ where the commutant of $H_{i}$ in $E_{8}$ is $G_{i}$. The singularity structure of the F-theory elliptic fibration is determined in terms of a Weierstrass model $Y^{2}=X^{3}+f X+g$ over $\mathcal{B}_{3}$, parameterized by $f$ and $g$, which are as before sections of line bundles $\mathcal{O}(-4 K), \mathcal{O}(-6 K)$ where $K$ is the canonical class of $\mathcal{B}_{3}$. The minimal group factors $G_{i}$ are determined by the minimal degrees of vanishing of $f, g$ as listed in table 1 , and in some cases distinguished by monodromy around the singular divisor $[22,56,83]$. Note that the Kodaira singularity dictates the physical gauge algebra only; different theories may have gauge groups that differ by a discrete factor that does not affect the algebra. In much of this paper we are somewhat cavalier about the distinction between gauge algebra and gauge group, but the reader should keep in mind that in most cases the only structure fixed by the local 


\begin{tabular}{|cc|c|c|}
\hline $\operatorname{deg} f$ & $\operatorname{deg} g$ & $\mathcal{G}_{i}$ & $\mathcal{H}_{i}$ \\
\hline 1 & 2 & $\mathfrak{s u}_{2}$ & $\mathfrak{e}_{7}$ \\
2 & 2 & $\mathfrak{s u}_{3}, \mathfrak{s u}_{2}$ & $\mathfrak{e}_{6}, \mathfrak{e}_{7}$ \\
2 & 3 & $\mathfrak{s o}_{8}, \mathfrak{s o}_{7}, \mathfrak{g}_{2}$ & $\mathfrak{s o}_{8}, \mathfrak{s o}_{9}, \mathfrak{f}_{4}$ \\
3 & 4 & $\mathfrak{e}_{6}, \mathfrak{f}_{4}$ & $\mathfrak{s u}_{3}, \mathfrak{g}_{2}$ \\
3 & 5 & $\mathfrak{e}_{7}$ & $\mathfrak{s u}_{2}$ \\
4 & 5 & $\mathfrak{e}_{8}$ & trivial \\
\hline
\end{tabular}

Table 1. The gauge algebra summands associated with group factors $G_{i}$ arising in $4 \mathrm{D}$ supergravity theory from divisors on which Weierstrass parameters $f, g$ vanish to various degrees, and the associated structure group factors for dual heterotic bundles.

singularity structure of the F-theory geometry is the gauge algebra.

The only gauge factors listed in table 1 are those that can be forced to arise from the structure of the F-theory base threefold, independent of the choice of elliptic fibration, in cases with a smooth heterotic dual. This is analogous to the generic gauge groups for 6 D F-theory models over Hirzebruch surfaces $\mathbb{F}_{m}$ [21], with for example a generic gauge group of $E_{6}$ over the curve of self-intersection -6 in $\mathbb{F}_{6}$. For more general F-theory models (i.e., those without smooth heterotic duals) there can be more complicated minimal gauge groups, some involving multiple gauge group factors - in analogy to the the general set of structures arising in maximally Higgsed 6D F-theory constructions, which can contain "non-Higgsable" matter [8]. In general, the gauge group in a particular model may be larger than the minimum group dictated by the structure of the base. For example, over some F-theory base threefolds it is possible to tune the Weierstrass coefficients $f, g$ to have an $A_{4}$ singularity corresponding to a gauge factor SU(5) over certain divisors, though this group does not arise as an automatic consequence of the geometry of any base corresponding to a singularity that arises in the generic elliptic fibration over that base. While a wide variety of models with different gauge groups can be tuned over each base, we are focused here on the minimal gauge group for each base.

In a number of places we will need to know the precise gauge algebra associated with given degrees of vanishing of $f, g$ over the divisors $\Sigma_{ \pm}$, including the effects of monodromy. As described in $[56,83]$, this can be determined by performing an expansion $f=f_{0}+$ $f_{1} z+f_{2} z^{2}+\cdots, g=g_{0}+g_{1} z+g_{2} z^{2}+\cdots$ around the divisor $D$ of interest, where $z$ is an algebraic coordinate that vanishes on $D$. Since the precise conditions that determine the monodromy are expressed differently in various places in the literature, we collect here a succinct summary of the possible situations. When $\operatorname{deg} f=2, \operatorname{deg} g=2$, the gauge algebra is $\mathfrak{s u}_{3}$ when $g_{2}$ is a perfect square, and $\mathfrak{s u}_{2}$ otherwise. Similarly, when $\operatorname{deg} f=3, \operatorname{deg} g=4$ the algebra is $\mathfrak{e}_{6}$ when $g_{4}$ is a perfect square, and $\mathfrak{f}_{4}$ otherwise. The case $\operatorname{deg} f=2 \operatorname{deg} g=3$ is somewhat more complicated; in this case, the algebra depends on the factorization properties of the cubic $X^{3}+f_{2} X+g_{3}$. If this cubic can be algebraically factorized into a product of three terms to the form $(X-a)(X-b)(X+(a+b))$ then the gauge algebra is $\mathfrak{s o}_{8}$, if it factorizes into the form $(X-a)\left(X^{2}+a X+b\right)$ the algebra is $\mathfrak{s o}_{7}$, 
and if it does not factorize algebraically, the algebra is $\mathfrak{g}_{2}$. We use these conditions in the analysis in several places in the remainder of the paper.

It is also worth noting that in the cases $T=2 c_{1}\left(B_{2}\right)$ that admit an $\mathrm{SO}(32)$ heterotic dual, the twist $T$ - and hence the base $\mathcal{B}_{3}$ on the F-theory side - is fixed uniquely for any $B_{2}$. The corresponding minimal gauge group is always $\mathfrak{s o}_{8}$, matching with the expectation from the heterotic side. This is shown in section 6.3

\subsection{Possible base surfaces for smooth heterotic/F-theory duals}

There are only a limited class of bases $B_{2}$ over which the elliptic fibration geometry is a smooth Calabi-Yau on the heterotic side. The set of complex base surfaces over which an elliptically fibered Calabi-Yau threefold exists can be classified according to the intersection structure of effective divisors on the base [8]. When the base contains a curve of selfintersection -3 or below, the total space of the elliptic fibration becomes singular, and in general the heterotic theory acquires an enhanced gauge group. Note that there are some special cases where an F-theory construction on a $\mathbb{P}^{1}$ bundle over a base surface with -3 curves can apparently exist without an extra nonabelian gauge group, though there is still no smooth heterotic dual in such cases as the dual Calabi-Yau geometry would be singular; an example of such a model is given in section 7.3. Our restriction to models with smooth heterotic duals means that we limit our analysis here to bases $B_{2}$ that only have effective curves of self-intersection -2 or above.

The set of bases $B_{2}$ that contain no curves of self-intersection -3 or below consist of the del Pezzo surfaces $d P_{n}$, given by $\mathbb{P}^{2}$ blown up at $n \leq 9$ points, the bases $\mathbb{F}_{0}=\mathbb{P}^{1} \times \mathbb{P}^{1}$ and $\mathbb{F}_{2}$ (which is a limit of $\mathbb{F}_{0}$ with a -2 curve), and the broader class of generalized del Pezzo surfaces, which are in general described as limits of del Pezzo surfaces containing curves of self-intersection -2 [28]. For each $n, 1<n<9$, there are generalized del Pezzo surfaces corresponding to limits of $d P_{n}$ with a set of -2 curves having an intersection structure corresponding to any proper subgraph of the extended Dynkin diagram $\hat{E}_{n}$ [84]. For $n=9$, the classification is slightly more complicated. There are 279 rational elliptic surfaces with different combinations of -2 curves, corresponding to generalized del Pezzo surfaces with $n=9$; these surfaces are classified in [85, 86]. Over each of the possible base surfaces $B_{2}$ there are a wide range of possible twists $T$ giving different geometries on the F-theory side. Each such geometry will correspond to a different class of bundles on the heterotic side on the Calabi-Yau describing an elliptic fibration over $B_{2}$. In principle, all possible $\mathrm{F}$-theory bases $\mathcal{B}_{3}$ with a smooth heterotic dual can be classified by determining all allowed twists $T$ for each del Pezzo and generalized del Pezzo (and for $\mathbb{F}_{0}=\mathbb{P}^{1} \times \mathbb{P}^{1}$ ).

\subsection{Heterotic/F-theory duals with toric base surfaces}

A particularly simple class of bases $B_{2}$ can be described using toric geometry. In [60], the complete set of toric bases for elliptic threefold fibrations was enumerated. Here we are only interested in those cases where all effective curves have self-intersection -2 or above, which restricts us to only 16 possible bases: the del Pezzo surfaces $d P_{n}$ with $0 \leq n \leq 3$, the surfaces $\mathbb{F}_{0}$ and $\mathbb{F}_{2}$, and 11 other toric generalized del Pezzo surfaces with various combinations of curves of self-intersection -2 . These 16 bases are listed in table 4 . 
Following standard methods in toric geometry [87, 88], we characterize the base $B_{2}$ by the toric fan, consisting of vectors $v_{0}, \ldots, v_{k+1} \in N_{2}=\mathbb{Z}^{2}$. This describes a base with $h_{1,1}\left(B_{2}\right)=k$. We can choose a basis in which $v_{0}=(1,0)$ and $v_{k+1}=(0,1)$. The coordinates of the remaining vectors defining $B_{2}$ can be written as $v_{i}=\left(x_{i}, y_{i}\right)$ in this basis. Any toric $\mathbb{P}^{1}$ bundle over $B_{2}$ can then be described in terms of a $3 \mathrm{D}$ toric fan

$$
\begin{aligned}
w_{0} & =(1,0,0) \\
w_{i} & =\left(x_{i}, y_{i}, t_{i}\right), 1 \leq i \leq k \\
w_{k+1} & =(0,1,0) \\
w_{k+2, k+3}=s_{ \pm} & =(0,0, \mp 1),
\end{aligned}
$$

where $s_{ \pm}$correspond to the divisors $\Sigma_{ \pm}$, and $w_{a}, 0 \leq a \leq k+1$, are $3 \mathrm{D}$ rays that project to $v_{a}$, with the third component $t_{i}$ parameterizing the twist $T$ that defines the $\mathbb{P}^{1}$ bundle. The vanishing of the third component of $w_{0}, w_{k+1}$ is a coordinate choice on $N_{3}=\mathbb{Z}^{3}$ used to eliminate two redundant degrees of freedom in the twist $T$. For convenience we will use indices $i \in\{1, \ldots, k\}, a \in\{0, \ldots, k+1\}$, and $\alpha \in\{0, \ldots, k+3\}$, and write $r=k+4$ for the total number of rays generating the $3 \mathrm{D}$ toric fan.

The toric language gives a simple description of the monomials available in the Weierstrass description of the F-theory model. If the set of $1 \mathrm{D}$ rays describing the toric threefold $\mathcal{B}_{3}$ are $w_{\alpha}, \alpha=0, \ldots, r-1$, then the monomials in $f$ are in one-to-one correspondence with the elements $m=(a, b, c) \in M=N^{*}$ of the dual lattice whose inner product with all of the $w_{\alpha}$ is not less than -4 ,

$$
\mathcal{F}=\left\{m \in N_{3}^{*}:\left\langle m, w_{\alpha}\right\rangle \geq-4, \forall \alpha\right\} .
$$

Similarly, the monomials in $g$ are associated with

$$
\mathcal{G}=\left\{m \in N_{3}^{*}:\left\langle m, w_{\alpha}\right\rangle \geq-6, \forall \alpha\right\} .
$$

As a simple example, for the case $\mathcal{B}_{3}=\mathbb{P}^{1} \times \mathbb{P}^{1} \times \mathbb{P}^{1}$, which is the trivial $\mathbb{P}^{1}$ bundle over $\mathbb{F}_{0}=\mathbb{P}^{1} \times \mathbb{P}^{1}$, the rays $w_{\alpha}$ are the basis vectors $( \pm 1,0,0),(0, \pm 1,0),(0,0, \pm 1)$, and the monomials in $f, g$ are the triplets $(a, b, c) \in \mathbb{Z}^{3}$ with $|a|,|b|,|c| \leq 4,6$.

For a toric F-theory base, we can compute the (anti)canonical class of $B_{2}$ directly from the toric description. There are two equivalence relations on the set of divisors $D_{a}$ associated with the rays $v_{a}$, giving

$$
\begin{aligned}
D_{0} & \sim \sum_{i=1}^{k}-x_{i} D_{i} \\
D_{k+1} & =\sum_{i=1}^{k}-y_{i} D_{i} .
\end{aligned}
$$

We have then

$$
-K_{2}=c_{1}\left(B_{2}\right)=\sum_{a=0}^{k+1} D_{a}=\sum_{i}\left(1-x_{i}-y_{i}\right) D_{i} .
$$


Similarly, we have

$$
-K_{3}=2 \Sigma_{-}-K_{2}+T,
$$

where $T=\sum t_{i} D_{i}$. As discussed below for more general $\mathrm{F}$-theory geometries with a heterotic dual, the formula (3.14) also follows straightforwardly from the definition of $\mathcal{B}_{3}$ given in (3.1) and the adjunction formula [19].

\section{F-theory constraints}

In this section we describe the geometric constraints on $\mathbb{P}^{1}$-bundle threefold bases in the class of models with smooth heterotic duals. In section 4.1 we describe constraints on the threefold geometry, and in section 4.2 we describe further constraints on the gauge group of the corresponding 4D supergravity theory and the extent to which it can be enhanced through "unHiggsing" by moving on the Calabi-Yau moduli space.

\subsection{Constraints on threefold base geometry}

The basic conditions on an F-theory threefold base geometry $\mathcal{B}_{3}$ are that there are no codimension one or codimension two loci with singularities worse than the $\mathfrak{e}_{8}$ singularity in the Kodaira classification. These conditions can be described in terms of constraints on the base geometry $B_{2}$ and twist $T$ describing $\mathcal{B}_{3}$ as a $\mathbb{P}^{1}$ bundle over $B_{2}$. We begin (section 4.1.1) with a brief overview of the general F-theory constraints, which are easy to make explicit in the toric context (section 4.1.2). In section 4.1.3, we use the general conditions to derive a set of local constraints on the twist $T$ associated with specific divisors in the base. The toric description of these constraints is given in section 4.1.4. In section 4.1.5, we derive a simple set of necessary conditions associated with the divisors $\Sigma_{ \pm}$. Combined with the constraints on $T$, this gives a set of conditions that are necessary, but not sufficient, for the existence of a good F-theory compactification geometry. In section 4.1.6, we include more general, nonlocal conditions associated with curves in $\mathcal{B}_{3}$, which give a set of sufficient conditions for an acceptable F-theory model, subject to issues from codimension three singularities and G-flux that we do not address here (see section 7.8 for some relevant aspects of G-flux for 4D F-theory compactifications).

In the analysis in this section we repeatedly use a basic result from algebraic geometry, which states that if an effective divisor $A$ on a surface $S$ has a negative intersection $A \cdot D<0$ with an irreducible effective divisor $D$ having negative self-intersection $D \cdot D<0$, then $A$ contains $D$ as a component, meaning that $A=D+X$ with $X$ effective. This means in particular that any section $s \in \mathcal{O}(A)$ must vanish on $D$. This result was used in [8] to identify the "non-Higgsable clusters" that classify the intersection structure of base surfaces $\mathcal{B}_{2}$ for 6D F-theory compactifications. More generally, an effective divisor may contain a number of rigid divisors $D_{i}$ with multiplicity $\gamma_{i}$ by repeated applications of the preceding rule

$$
A=\sum_{i} \gamma_{i} D_{i}+X, \quad(X \text { effective }) .
$$

When such a decomposition is carried out over the rational numbers $\gamma_{i} \in \mathbb{Q}$, it is called the Zariski decomposition of $A$. While in higher dimensions the Zariski decomposition can 
be more subtle, for surfaces the computation of the terms $\gamma_{i} D_{i}$, known as the base locus of $A$ is straightforward. For example, if $D$ is a curve of self-intersection $D \cdot D=-2$, and $A \cdot D=-4$, then $A=2 D+X$ with $X$ effective, $X \cdot D=0$.

In a number of places in this section we focus on curves in $\mathcal{B}_{3}$ of the form $C=\Sigma_{ \pm} \cap D$, where $D$ is a divisor on $\mathcal{B}_{3}$ pulled-back from a corresponding divisor in the base surface $B_{2}$. We will generally use $C$ for the curve in $\mathcal{B}_{3}$, while $D$ can refer either to the divisor in $\mathcal{B}_{3}$ or in $B_{2}$, depending on context.

\subsubsection{General constraints from F-theory geometry}

We begin with a general statement of the F-theory constraints that hold for any geometry. For a good F-theory model to exist on a base $\mathcal{B}_{3}$ there must be a Calabi-Yau fourfold that is elliptically fibered over $\mathcal{B}_{3}[21,22,46]$. As described in section 3.1 , when the Weierstrass coefficients $f, g$ and the discriminant $\Delta=4 f^{3}+27 g^{2}$ vanish on a divisor in $\mathcal{B}_{3}$, the corresponding $4 \mathrm{D}$ supergravity theory gets a nonabelian gauge group contribution depending upon the Kodaira type of the corresponding singularity in the elliptic fibration. When the vanishing degrees of $(f, g, \Delta)$ reach or exceed $(4,6,12)$ on a divisor, the fibration becomes too singular to admit a Calabi-Yau resolution. Thus, a constraint on $\mathcal{B}_{3}$ is that $-n K_{3}$ must admit a section of vanishing degree $<n$ for $n=4$ or 6 on any irreducible effective divisor $D$. Similarly, $f, g$ cannot vanish to orders 4, 6 on any curve, or the curve would need to be blown up, giving a different base structure, for a Calabi-Yau resolution of the singular elliptic fibration to exist. This provides a strong set of constraints on bases $\mathcal{B}_{3}$ that admit good F-theory models. The constraint on codimension three loci (points) on the base is less clear; if the degrees of $f, g$ reach 8,12 on a point then the point must be blown up for a good Calabi-Yau resolution. On the other hand, if the degrees of vanishing reach 4,6 on a codimension three locus but do not exceed 8,12 then the model may be problematic yet cannot be blown up directly [89]. We focus in this paper on the constraints associated with codimension one and two loci, associated with gauge groups and matter content in the low-energy theory. We include therefore in our analysis models with codimension 3 singularities, leaving the resolution of the status of these models to future work. Codimension three singularities are discussed further in section 7.8.

\subsubsection{Constraints for toric bases}

The F-theory constraints described above are particularly simple to describe for toric Ftheory bases $\mathcal{B}_{3}$ using the explicit description of the Weierstrass monomials as elements of the dual lattice, as described in the previous section. ${ }^{11}$ The degrees of vanishing of $f, g$ on the divisor $D_{\alpha}$ associated with the ray $w_{\alpha}$ are given by

$$
\operatorname{deg}_{D_{\alpha}} f=\min _{m \in \mathcal{F}}\left\langle m, w_{\alpha}\right\rangle+4, \quad \operatorname{deg}_{D_{\alpha}} g=\min _{m \in \mathcal{G}}\left\langle m, w_{\alpha}\right\rangle+6 .
$$

These are easily computed for any given base and divisor. For a good F-theory base, these degrees cannot both reach or exceed 4,6 on any divisor $D_{\alpha}$. When the degrees are nonzero,

\footnotetext{
${ }^{11}$ Related constraints in the toric language of "tops" were described in [91].
} 
they indicate the presence of a generic gauge algebra factor on $D_{\alpha}$, according to table 1 . The degrees of vanishing of $f, g$ on the toric curve $D_{\alpha} \cap D_{\beta}$ are given by

$$
\operatorname{deg}_{D_{\alpha} \cap D_{\beta}} f=\min _{m \in \mathcal{F}}\left\langle m, w_{\alpha}+w_{\beta}\right\rangle+8, \quad \operatorname{deg}_{D_{\alpha} \cap D_{\beta}} g=\min _{m \in \mathcal{G}}\left\langle m, w_{\alpha}+w_{\beta}\right\rangle+12 .
$$

Again, these degrees cannot both reach or exceed 4,6 on any curve or the F-theory base must be blown up along that curve to give a new base. When these degrees reach or exceed 1,2 along a given curve they generally indicate the presence of $\mathcal{N}=2$ matter transforming under gauge groups carried on the divisors $D_{\alpha}, D_{\beta}$, in analogy to the $6 \mathrm{D}$ situation. In some toric $4 \mathrm{D}$ cases, however, there are no such nonabelian gauge groups. An example of such a situation is described explicitly in section 7.2 , with $\mathcal{B}_{3}$ a $P^{1}$ bundle over $B_{2}=\mathbb{F}_{1}$. Such codimension two singularities may simply represent cusps where the discriminant becomes singular, as occurs in 6D compactifications (see e.g. [90]), or may in some cases represent matter charged under $\mathrm{U}(1)$ gauge factors.

\subsubsection{F-theory bounds on twists}

We now specialize to the class of F-theory geometries that have smooth heterotic duals as described in section 3. In this case, the relation (3.14) derived above in the toric context holds more generally from the adjunction formula applied to $\mathcal{B}_{3}$ defined in (3.1)

$$
-K_{3}=2 \Sigma_{-}-K_{2}+T .
$$

Writing the Weierstrass functions $f, g$ locally in a region around the locus $\Sigma_{-}$defined by the coordinate $z=0$, where $\Sigma_{+}$is at $z=\infty$, we have

$$
\begin{aligned}
& f=f_{0}+f_{1} z+f_{2} z^{2}+\cdots f_{8} z^{8} \\
& g=g_{0}+g_{1} z+g_{2} z^{2}+\cdots g_{12} z^{12} .
\end{aligned}
$$

The term $f_{k} z^{k}$ vanishes to order $k$ on $\Sigma_{-}$and to order $8-k$ on $\Sigma_{+}$, and $f_{k}$ is a section of $\mathcal{O}\left(-4 K_{3}-k \Sigma_{-}-(8-k) \Sigma_{+}\right)$. Similarly, $g_{k} z^{k}$ vanishes to order $k$ on $\Sigma_{-}$and to order $12-k$ on $\Sigma_{+}$, and $g_{k}$ is a section of $\mathcal{O}\left(-6 K_{3}-k \Sigma_{-}-(12-k) \Sigma_{+}\right)$. Thus, $f_{k}$ and $g_{k}$ are sections of $\mathcal{O}\left(-4 K_{2}-(4-k) T\right)$ and $\mathcal{O}\left(-6 K_{2}-(6-k) T\right)$ respectively. We can use this fact to determine constraints on the possible twists $T$ compatible with any particular base $B_{2}$.

A set of necessary conditions on $T$ can be determined by imposing the condition that $f, g$ should not vanish to orders 4,6 on any curve $C$ in $\mathcal{B}_{3}$ that is of the form $\Sigma_{-} \cap D$, where $D$ is associated with an irreducible effective divisor on $B_{2}$. We consider the various possibilities depending upon the self-intersection of $D$ in $B_{2}$, using the fact that $\left(K_{2}+D\right) \cdot D=-2$ when $D$ is a rational curve. We focus on divisors $D$ with non-positive self-intersection $D \cdot D \leq 0$, since determining conditions on these divisors is sufficient to bound the total number of twists $T$ over any base $B_{2}$. Note that, as shown in [8], any higher genus divisor of negative self-intersection in the base $B_{2}$ gives a singular elliptic fibration that cannot be resolved to a Calabi-Yau, so it is sufficient to restrict attention to rational curves $D$.

We begin with the case $D \cdot D=-2$, where $K_{2} \cdot D=0$. Consider then the intersection

$$
\left(-n K_{2}-(n-k) T\right) \cdot D=-(n-k) T \cdot D, k<n .
$$


When $T \cdot D>0$, this intersection is negative, and $\left(-n K_{2}-(n-k) T\right)$ contains $D$ as a component for $n=4,6$ when $0 \leq k<n$. This means that the sections $f_{k}, g_{k}$ must vanish on $D$. If $T \cdot D \geq 2$, then $f_{k}, g_{k}$ must vanish on $D$ at least to order $4-k, 6-k$ respectively, which would mean that $f, g$ vanish to degrees 4,6 on $C$. Thus, the twist must satisfy $T \cdot D<2$ for any rational curve $D$ in the base having self-intersection $D \cdot D=-2$. A similar argument for $\Sigma_{+}$shows that $T \cdot D>-2$, so $|T \cdot D| \leq 1$ for any -2 curve in $B_{2}$.

Now, consider the case $D \cdot D=-1$. In this case, $-K_{2} \cdot D=1$, so we have $\left(-n K_{2}-\right.$ $(n-k) T) \cdot D=n-(n-k) T \cdot D$. This is less than or equal to $-(n-k)$ for all $k<n=4,6$ when $T \cdot D>6$, which would force $f, g$ to vanish on $D \cap \Sigma_{-}$to degrees 4 , 6 , with a similar constraint with the opposite sign for $\Sigma_{+}$, so we have a bound in this case of $|T \cdot D| \leq 6$. (For example, if $T \cdot D=6$, then $\left(-6 K_{2}-T\right) \cdot D=0$, so $g_{5}$ need not vanish on $D$, though all other $f_{k}, g_{k}$ vanish on $D$ to degree $n-k$.)

Similar reasoning shows that analogous constraints hold for curves of self-intersection 0 and for curves of more negative self-intersection; the complete set of constraints for the twist over any rational curve of (non-positive) self-intersection $-n$ is

$$
\begin{array}{rlr}
n=0: & |T \cdot D| \leq 12 \\
n=1: & |T \cdot D| \leq 6 \\
6 \geq n \geq 2: & |T \cdot D| \leq 1 . \\
n \geq 7: & T \cdot D=0 .
\end{array}
$$

We only need the results for $n \leq 2$ in this paper.

These bounds provide strong constraints on the twists that are allowed for a $\mathbb{P}^{1}$ bundle over any base $B_{2}$. As promised in section 1, these reduce the problem of identifying all smooth $\mathrm{F}$-theory bases $\mathcal{B}_{3}$ with smooth heterotic dual geometries to a finite enumeration problem, since the curves of negative or 0 self-intersection in the base $B_{2}$ form a connected set. We summarize the results of a complete enumeration of all twists over toric bases with smooth heterotic duals in section 9 .

\subsubsection{Toric bounds on twists}

The bounds on twists can be seen explicitly in the toric context. We can identify the bounds on the twist $t_{i}$ over the divisor $D_{i}$ associated with a given base ray $v_{i}$ by considering the local geometry of the $\mathbb{P}^{1}$ bundle over the sequence of rays $v_{i-1}, v_{i}, v_{i+1}$. (If $i=0$ or $i=k+1$, we replace $i-1$ or $i+1$ with $k+1$ or 0 respectively in the obvious fashion to respect the cyclic ordering of rays). We can choose a basis for $N_{2}$ so that $v_{i-1}=(1,0)$ and $v_{i}=(0,-1)$ (note that this is a different choice of basis than that used in (3.5)-(3.8)). Associated with the three $2 \mathrm{D}$ rays $v_{i-1}, v_{i}, v_{i+1}$ there are twists $t_{i-1}, t_{i}, t_{i+1}$, associated with the extension of the corresponding $3 \mathrm{D}$ rays $w_{i-1}, w_{i}, w_{i+1}$ in the third dimension (3.6). In the $3 \mathrm{D}$ toric lattice $N_{3}$ we can perform a linear transformation taking

$$
w_{i-1}=\left(1,0, t_{i-1}\right) \rightarrow \tilde{w}_{i-1}=(1,0,0), \quad w_{i}=\left(0,-1, t_{i}\right) \rightarrow \tilde{w}_{i}=(0,-1,0) .
$$

Since we are assuming that the base $B_{2}$ is smooth, the third ray has the form $w_{i+1}=$ $\left(-1,-n, t_{i+1}\right)$, where the integer $-n$ is the self-intersection of the divisor $D_{i}[87]$. The 
linear transformation (4.12) takes

$$
w_{i+1} \rightarrow \tilde{w}_{i+1}=\left(-1,-n, \tilde{t}_{i}\right), \text { where } \tilde{t}_{i}=t_{i-1}+t_{i+1}-n t_{i} .
$$

The parameter $\tilde{t}_{i}=T \cdot D_{i}$ determines the nontrivial part of the twist around the ray $v_{i}$, and can be constrained geometrically depending upon $n$, to reproduce the conditions (4.8)(4.11). As in the general situation described in the previous subsection, the strongest constraint on the twist component $\tilde{t}_{i}$ comes from the condition that $f, g$ do not have degrees 4,6 on the curves associated with $w_{i}+s_{ \pm}$. Let us assume that $\tilde{t}_{i} \geq 0$. Then we have the following constraints on the monomials associated with $m=(a, b, c) \in N_{3}^{*}$ for $f, g$ with $B=4,6$

$$
\begin{aligned}
\left\langle m, \tilde{w}_{i-1}\right\rangle & \geq-B \quad \rightarrow \quad a \geq-B \\
\left\langle m, \tilde{w}_{i}\right\rangle & \geq-B \quad \rightarrow \quad b \leq B \\
\left\langle m, s_{-}\right\rangle & \geq-B \quad \rightarrow \quad c \geq-B \\
\exists\left\langle m, \tilde{w}_{i}+s_{-}\right\rangle & <-B \quad \rightarrow \quad b-c>B \\
\left\langle m, \tilde{w}_{i+1}\right\rangle & \geq-B \quad \rightarrow \quad-a-n b+\tilde{t}_{i} \geq-B .
\end{aligned}
$$

All of these inequalities except (4.17) must be satisfied for all monomials in $\mathcal{F}, \mathcal{G}$. Inequality (4.17) on the other hand, need only be satisfied by at least one point $m$, to avoid having a 4,6 singularity on $w_{i}+s_{-}$. But this means that there must be at least one integral point satisfying all of these inequalities. The second through fourth inequalities define a simple triangle in the $b$-c plane within which any solutions must lie (figure 2). The final constraint imposes a condition that restricts the solutions within this triangle. This constraint is weakest when $a$ is maximally negative, so if there are any solutions in $b, c$ for any $a$ they will also be acceptable for $a=-B$. Therefore, we need only ask whether there can exist any solutions of the form $(-B, b, c)$ to inequalities (4.15)-(4.18). For $n=0$, (4.18) becomes $\tilde{t}_{i} c \geq-2 B$, and since $c<0$ from (4.17) and (4.15), we need $\tilde{t}_{i} \leq 2 B$ for a solution of all the inequalities to exist. This is weakest for $B=6$, so we have the constraint $\tilde{t}_{i} \leq 12$ when $n=0$. For $n=1$, (4.18) becomes $b \leq \tilde{t}_{i} c+2 B$, which combined with (4.17) becomes $c+B<\tilde{t}_{i} c+2 B$, so $\left(\tilde{t}_{i}-1\right)(-c)<B$ and since $-c>0$ we need $\tilde{t}_{i} \leq 6$. Finally, for $n=2$, (4.18) becomes $2 b \leq \tilde{t}_{i} c+2 B$, which combined with (4.17) becomes $2 c<\tilde{t}_{i} c$, and since $c<0$ this implies $\tilde{t}_{i} \leq 1$. To summarize, we have reproduced the constraints $(4.8)-(4.11)$ in the toric context

$$
\begin{array}{ll}
n=0: & \left|\tilde{t}_{i}\right| \leq 12 \\
n=1: & \left|\tilde{t}_{i}\right| \leq 6 \\
n \geq 2: & \left|\tilde{t}_{i}\right| \leq 1 .
\end{array}
$$

The constraints on coefficients in $g(B=6)$ for $n=2, \tilde{t}_{i}=1$ are shown in figure 2 .

\subsubsection{General constraints on geometry from $f, g$ on divisors}

In section 4.1.3 we used curves of the form $C=\Sigma_{ \pm} \cap D$ in the base $\mathcal{B}_{3}$ to determine bounds on the individual components $T \cdot D$ of the twist $T$ parameterizing the $\mathbb{P}^{1}$ bundle over $B_{2}$. We now describe more general constraints on $T$ associated directly with the divisors $\Sigma_{ \pm}$. 


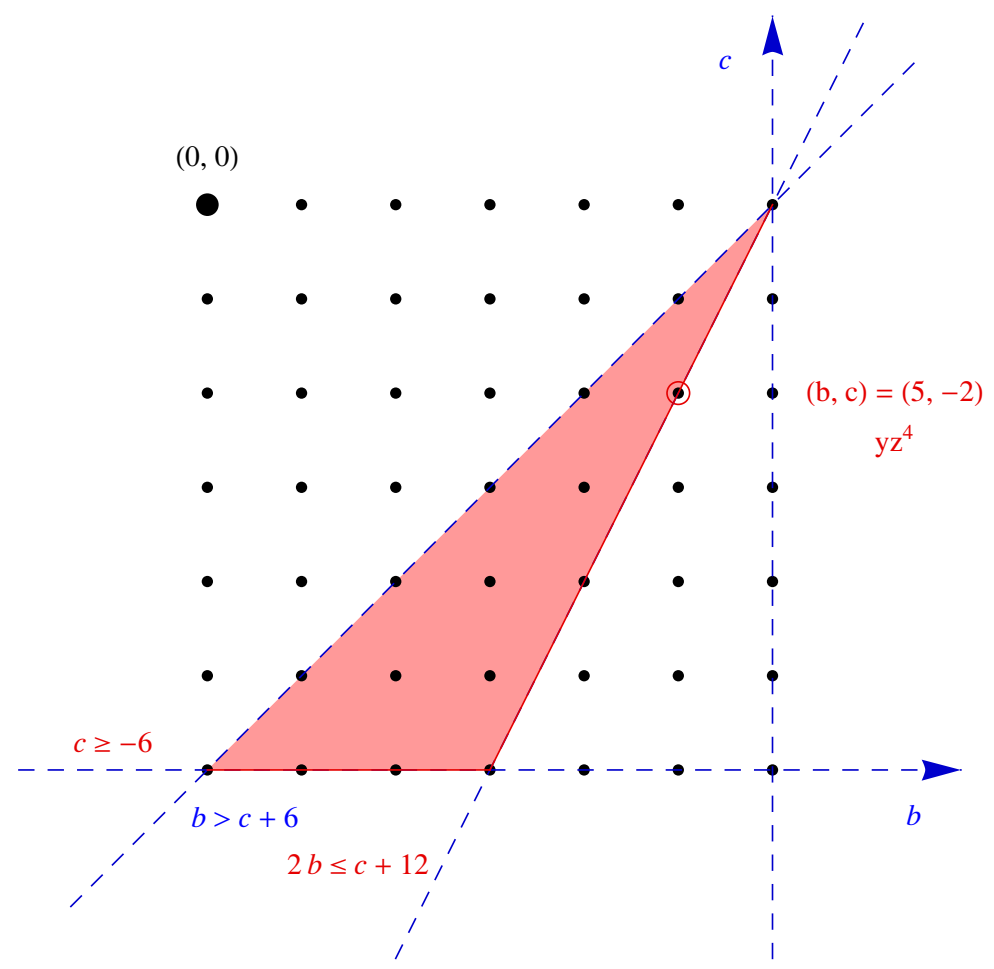

Figure 2. Constraints imposed on dual monomials in the Weierstrass function $g$ parameterized by $a, b, c$ in the toric description of a twist $\tilde{t}_{i}=1$ over a curve of self-intersection $-n=-2$; the depicted constraints on $b, c$ correspond to weakest conditions, which hold at $a=-B=-6$. For a smooth F-theory geometry, at least one monomial in the shaded region (not including the boundary at $b>c+6$, or the corresponding region for $f(B=4)$ must be nonzero. The circled point $(5,-2)$ corresponds to the monomial $y z^{4}$ in coordinates where $z=0$ corresponds to $\Sigma_{-}$and $y=0$ corresponds to $D_{i}$. This point is relevant in ruling out gauge algebra factors $\mathfrak{e}_{7}, \mathfrak{e}_{8}$ on $\Sigma_{-}$ under these conditions (section 6.4).)

Note that the divisors $D$ on the base cannot give further constraints on $T$ since $f, g$ cannot vanish on $D$ to higher degree than they do on the corresponding curve in $B_{2}$. This follows from the fact that $f_{4}, g_{6}$ are sections of $-4 K_{2},-6 K_{2}$ respectively. Thus, we need only consider constraints associated with the degrees of vanishing of $f, g$ on the divisors $\Sigma_{ \pm}$.

As described in section 4.1.3, the components $f_{k}, g_{k}$ of the discriminant locus that vanish to degree $k$ on $\Sigma_{-}$are associated with sections of the line bundles $\mathcal{O}\left(-4 K_{2}-(4-k) T\right)$ and $\mathcal{O}\left(-6 K_{2}-(6-k) T\right)$ respectively. In particular, $g_{5}$ is a section of $\mathcal{O}\left(-6 K_{2}-T\right)$. If $-6 K_{2}-T$ is effective, then this line bundle admits a section, so generically $g_{5}$ is nonzero and $g$ does not vanish to degree 6 on $\Sigma_{-}$. On the other hand, if $-6 K_{2}-T$ is not effective then there are no sections and $g_{5}=0$. Furthermore, if $-6 K_{2}-T$ is not effective then $-6 K_{2}-n t$ cannot be effective for $n \geq 2$, since

$$
n\left(-6 K_{2}-T\right)=-6 K_{2}-n t+(n-1)\left(-6 K_{2}\right)
$$

and $-6 K_{2}$ is effective, as is any positive combination of effective divisors. Similarly, if 
$-6 K_{2}-T$ is not effective then $-4 K_{2}-m t$ cannot be effective for $m \geq 1$ since

$$
m\left(-6 K_{2}-T\right)=-4 K_{2}-m t+(6 m-4)\left(-K_{2}\right)
$$

where again the last term is effective. This shows that $-6 K_{2}-T$ is effective if and only if $f, g$ do not vanish to degrees 4,6 on $\Sigma_{-}$. A parallel argument shows that $-6 K_{2}+T$ is effective if and only if $f, g$ do not vanish to degrees 4,6 on $\Sigma_{+}$.

From these considerations we can distinguish several possible configurations of allowed geometries on the F-theory side

A) One possibility is that

$$
T=-6 K_{2}
$$

In this case all coefficients of $f_{k}, g_{k}$ vanish up to but not including degrees $(4,5)$.

B) If $T \neq-6 K_{2}$ then $g_{5}$ is not a constant and must vanish on some curves $D$ in $B_{2}$. To avoid having $f, g$ both vanish to degrees 4,6 on the associated curve $C=\Sigma_{-} \cap D$ in $\mathcal{B}_{3}$ at least one other coefficient $f_{k \leq 3}, g_{k \leq 4}$ must be nonvanishing. But by a parallel argument to the above this means that

$$
-4 K_{2}-T \text { is effective. }
$$

C) If there is any curve $D$ in the base with self-intersection $D \cdot D=-2$, where the associated component of the twist is $T \cdot D=+1$, then by the analysis of section 4.1.3 we know that $f_{3}, g_{5}$ both vanish on $D$. This means that one of $f_{k \leq 2}, g_{k \leq 4}$ must be nonvanishing. Again, a parallel argument to the above means that in this case

$$
\exists D: D \cdot D=-2, T \cdot D=+1 \Rightarrow-3 K_{2}-T \text { is effective. }
$$

Any good F-theory base geometry must satisfy these conditions and must fit into one of the 3 categories (A-C). A similar set of conditions hold for $\Sigma_{+}$where the sign is changed for $T$ on all equations.

\subsubsection{General constraints on geometry from $f, g$ on curves}

The conditions (A-C) derived in section 4.1.5, along with the local twist conditions (4.8)(4.11), give a set of necessary conditions that must be satisfied for any F-theory compactifications on a space $\mathcal{B}_{3}$ that is a $\mathbb{P}^{1}$ bundle over a base $B_{2}$ without divisors of self-intersection below -2 . These conditions are not, however, sufficient. While the conditions derived in the preceding subsection are both necessary and sufficient for $f, g$ to be well-behaved on divisors, the local constraints on twist components $T \cdot D$ are not sufficient to guarantee that $f, g$ are well-behaved on all curves $C=\Sigma_{-} \cap D$ with $D$ a divisor in $B_{2}$. In general, "nonlocal" effects from other divisors can limit the range of allowed twists more stringently than the local conditions (4.8)-(4.11). Note, however, that there are no further constraints associated with curves formed from the intersection of two divisors $D_{i}, D_{j}$ since the vanishing on such curves cannot be greater than at the corresponding points in $B_{2}$. 
As an example of a further constraint following from the interaction between twists on different divisors, consider a base $B_{2}$ that contains two divisors $D_{1}, D_{2}$ with $D_{1} \cdot D_{1}=$ $D_{2} \cdot D_{2}=-2, D_{1} \cdot D_{2}=1$. While the bound (4.10) seems to allow $T \cdot D_{1}=T \cdot D_{2}=1$, if this were to hold then $f, g$ would vanish to degrees 4,6 on $D_{1} \cap \Sigma_{-}, D_{2} \cap \Sigma_{-}$. This can be seen by considering (as usual, for $n=4,6$ and $0 \leq k<n) Q=\left(-n K_{2}-(n-k) T\right.$ ), which satisfies

$$
\begin{aligned}
& Q \cdot D_{1}=-(n-k) T \cdot D_{1}=-(n-k)<0 \\
& Q \cdot D_{2}=-(n-k) T \cdot D_{2}=-(n-k)<0 .
\end{aligned}
$$

We then have a decomposition (4.1) of $Q$ of the form

$$
Q=\gamma D_{1}+\eta D_{2}+X
$$

with $X \cdot D_{1} \geq 0, X \cdot D_{2} \geq 0$, so $(\eta-2 \gamma) \leq-(n-k),(\gamma-2 \eta) \leq-(n-k)$, from which it follows that $\eta \geq n-k, \gamma \geq n-k$.

A general statement of the sufficient conditions on $T$ for $f, g$ to be well-behaved on all curves $D \cap \Sigma_{ \pm}$(and all divisors $\Sigma_{-}$) is that for each divisor $D$ in $B_{2}$, there is at least one $n, k$ $(n=4$ or $6, k<n)$ so that $Q_{n, k}=-n K_{2}-(n-k) T$ has a decomposition of the form (4.1) with $\gamma_{D}<k$. This condition is automatically satisfied for any base $B_{2}$ without -2 curves, since for a -1 curve $D$ as long as $-6 K_{2}-T$ is effective, $Q_{6,5} \cdot D=6-T \cdot D \geq 0$ for $T \cdot D \leq 6$, so $Q_{6,5}$ is effective and has sections that do not vanish on any -1 curves $D$ in the base.

This shows that while the conditions described in section 4.1.5 and (4.8)-(4.11) are sufficient for a model to have acceptable $f, g$ on all divisors and curves when the base is del Pezzo (or $\mathbb{P}^{2}$ or $\mathbb{F}_{0}$ ) with no -2 curves, when the base has -2 curves the more general conditions stated above must be included to give a set of sufficient conditions.

In formulating these conditions, note again that we have not considered potential problems with codimension 3 singularities or G-flux, which may make a geometry unsuitable for F-theory compactification even when the sufficient conditions discussed here are satisfied. These other issues are deferred to future work. In specific, however, note that any curve on which $f, g$ vanish to degrees 4,5 but are not constants will generically have points where $f, g$ vanish to degrees 4,6 , which are of codimension 3 in the full base $\mathcal{B}_{3}$.

\subsection{Constraints on gauge enhancement}

The constraints described so far limit the possible geometries that can be used for Ftheory compactification, and for any given geometry impose a minimum gauge group that cannot be broken without changing the F-theory base $B_{2}$. In some situations, the vanishing conditions on $f, g$ also impose constraints that limit the extent to which gauge group factors in the effective supergravity theory can be enhanced by "unHiggsing" matter fields to form larger gauge groups. These constraints give nontrivial limitations on bundle structure in the dual heterotic picture. We consider two specific types of such constraints. In the first type, generic $\mathrm{SU}(2)$ and $\mathrm{SU}(3)$ gauge groups are constrained from being enhanced to $\mathrm{SU}(N)$ with $N>3$; in the second type, constraints are associated with codimension two loci in the F-theory picture. In both cases, the restriction on enhancement is related to the absence of sufficient matter to represent a Higgsed phase of a theory with higher symmetry. 
Note that while the bounds considered here on gauge enhancement are a consequence of the geometry, it is possible that in some cases G-flux may decrease the size of the gauge group, as discussed further in later sections. This would not affect the upper bounds described in this section.

\subsubsection{Constraints on $\mathrm{SU}(2)$ and $\mathrm{SU}(3)$ enhancement}

One unusual feature of the gauge groups $\mathrm{SU}(2)$ and $\mathrm{SU}(3)$ is that they can be realized in two separate ways in F-theory, associated with two different Kodaira singularities. There is a standard $A_{N-1}$ realization of $\mathrm{SU}(N)$ where $f$ and $g$ do not vanish on a given divisor but $\Delta$ vanishes to order $N$ (an $I_{N}$ singularity in the Kodaira classification). Another realization of $\mathrm{SU}(2)$ arises when $f, g, \Delta$ vanish to degrees $1,2,3$ and $\mathrm{SU}(2)$ or $\mathrm{SU}(3)$ can be realized when $f, g, \Delta$ vanish to degrees 2, 2, 4 (Type III and IV singularities in the Kodaira classification). While type III and IV singularities are in one sense simply special limit points on the loci of type $I_{2}$ and $I_{3}$ singularities, their physical properties are rather different. When $\mathfrak{s u}_{2}$ or $\mathfrak{s u}_{3}$ gauge algebras are forced to exist on a divisor $D$ by type III or type IV singularities in the F-theory geometry, there is no way to tune the Weierstrass moduli to realize any $\operatorname{SU}(N)$ gauge group with $N>3$ on $D$ since this can only happen from a type $I_{N}$ singularity where $f, g$ do not vanish on $D$. This means that for many theories with generic $\mathrm{SU}(2)$ or $\mathrm{SU}(3)$ gauge symmetries there is in principle no branch of the theory with enhanced $\mathrm{SU}(N)$ gauge symmetry (particularly no SU(5) gauge symmetry).

As a concrete example of where this constraint is relevant, we begin by considering the $6 \mathrm{D}$ case of $\mathrm{F}$-theory compactification on $\mathbb{F}_{3}$. In this case, the base $\mathcal{B}_{2}=\mathbb{F}_{3}$ contains a divisor $\Sigma$ with self-intersection $\Sigma \cdot \Sigma=-3$ on which $f, g, \Delta$ necessarily vanish to degrees 2 , 2,4 . The resulting $\mathrm{SU}(3)$ gauge group in the corresponding $6 \mathrm{D}$ supergravity theory cannot be enhanced to $\mathrm{SU}(4)$ or any higher $\mathrm{SU}(N)$ by tuning the Weierstrass moduli to get an $A_{N-1}$ singularity. This is clear in the low-energy $6 \mathrm{D}$ theory, as there is no matter charged under the $\mathrm{SU}(3)$, such as would arise under Higgsing from a larger gauge group. As we discuss in section 7 , this corresponds in the heterotic dual picture to a constraint on how the associated bundle over K3 can be decomposed.

A similar constraint occurs for a wide range of $4 \mathrm{D}$ F-theory compactifications. In many $4 \mathrm{D}$ compactifications the structure of the base $\mathcal{B}_{3}$ is such that $f, g, \Delta$ are forced to vanish to degree $1,2,3$ or $2,2,4$, giving rise to a non-Higgsable $\mathrm{SU}(2)$ or $\mathrm{SU}(3)$ gauge group. In such cases these gauge groups cannot be enhanced to higher $\mathrm{SU}(N)$ with $N>3$ anywhere in the moduli space, though the $\mathrm{SU}(2)$ gauge factors can generally be enhanced to $\mathrm{SU}(3)$ by tuning moduli. We describe some specific examples where these kinds of constraints appear in section 7 .

\subsubsection{Constraints from codimension two loci}

In another class of situations, the extent to which a gauge group factor can be enhanced is limited by the degrees of vanishing of $f, g, \Delta$ on a locus of codimension 2. This occurs when the enhancement of the factor $G$ on a divisor $D$ to a given Kodaira singularity type automatically raises the degrees of vanishing of $f, g, \Delta$ on a codimension two locus to $4,6,12$ or beyond. 
In six dimensions, constraints of this type only appear for F-theory constructions without smooth heterotic duals. A simple example is when the base $\mathcal{B}_{2}$ contains two effective irreducible divisors $C_{1}, C_{2}$ with self and mutual intersections $C_{1} \cdot C_{1}=-2, C_{2} \cdot C_{2}=$ $-3, C_{1} \cdot C_{2}=1$. In such a situation, the $6 \mathrm{D}$ theory has a non-Higgsable gauge group with Lie algebra summands $\mathfrak{s u}_{2} \oplus \mathfrak{g}_{2}$, and there is matter charged under both groups, as described in [8]. While naively the $G_{2}$ factor can be tuned to an $E_{6}$ or $F_{4}$ by increasing the degrees of vanishing of $f$ and $g$ on $C_{2}$ to 3,4 , doing this raises the degrees of vanishing of $f, g$ to 4,6 on the intersection point $C_{1} \cdot C_{2}$, leading to a point in the base that must be blown up. Writing the Weierstrass coefficients explicitly, in a coordinate system where $z, w$ vanish on $C_{1}, C_{2}$ respectively we have $f=a z w^{2}+\mathcal{O}(z, w)^{4}, g=b z^{2} w^{3}+\mathcal{O}(z, w)^{6}$, from which the above conclusions follow directly.

While this kind of enhancement constraint only arises for 6D F-theory models that do not have heterotic duals, in $4 \mathrm{D}$ the issue is much more general. One key class of examples, which we discuss further in section 6 , are $4 \mathrm{D}$ compactifications of F-theory that have heterotic duals violating the base-point free condition (see section 5.3). In such situations an $F_{4}$ symmetry can have an obstruction to enhancement to an $E_{6}, E_{7}$ or $E_{8}$ on the F-theory side as such an enhancement would lead to a 4,6 vanishing of $f, g$ on a codimension two locus.

\section{$5 \quad$ Heterotic constraints}

As noted in section 1, the compactification of the $E_{8} \times E_{8}$ heterotic string gives rise to a number of consistency constraints, linking the topological data of the Calabi-Yau threefold with that (i.e., Chern classes) of the vector bundles $V_{1}, V_{2}$. We will be interested here in these bounds on topology, as well as in the conditions for supersymmetric $\mathcal{N}=1$ heterotic vacua.

We consider a pair $\left(V_{1}, V_{2}\right)$ of vector bundles on a Calabi-Yau threefold with structure groups $H_{i} \subseteq E_{8}, i=1,2$, which break each $E_{8}$ factor to the commutant $G_{i}$ of $H_{i}$ in $E_{8}$. The bundles must satisfy the topological constraints

$$
\begin{aligned}
& c_{1}\left(V_{1}\right) \equiv c_{1}\left(V_{2}\right) \equiv 0(\bmod 2) \\
& \operatorname{ch}_{2}\left(T X_{3}\right)-\operatorname{ch}_{2}\left(V_{1}\right)-\operatorname{ch}_{2}\left(V_{2}\right)+[W]_{\mathrm{eff}}=0
\end{aligned}
$$

The first of these conditions is equivalent to the vanishing of the second Steifel-Whitney class of the bundles $V_{i}$, a necessary condition for the existence of spinors; in the case of irreducible principal bundles, this reduces to the condition $c_{1}=0$ (see the discussion in section 2.2.2). Henceforth in this paper we focus attention on irreducible bundles and take $c_{1}=0$. The second constraint on the second Chern characters of the bundles is the familiar 10D anomaly cancellation condition we have already encountered in section 3.1 in the context of heterotic/F-theory duality. The last term in (5.2) is a non-perturbative contribution arising from NS5-branes (equivalently M5 branes, in heterotic M-theory), where $[W]_{\text {eff }}$ denotes the total class of effective curves wrapped by 5 -branes. In this work, we will not include 5-branes wrapping curves in the base $B_{2}$ (i.e., degenerations of the bundle corresponding to sheaves supported over curves) and as a result, any possible term $[W]_{\text {eff }} \neq 0$ will not affect (3.3), the definition of $\eta$ given in section 3.1. In some cases, 
non-perturbative effects in the form of 5-branes wrapping the elliptic fibers may be present (see [19] for a discussion of such heterotic 5-branes and G-flux).

For a supersymmetric vacuum, the vanishing of the 10-dimensional gaugino variation requires each bundle $V_{i}$ to satisfy the well-known "Hermitian-Yang-Mills" equations [1]

$$
F_{a b}=F_{\bar{a} \bar{b}}=0, \quad g^{a \bar{b}} F_{a \bar{b}}=0
$$

The first half of these conditions, namely the vanishing of $F^{2,0}=F^{0,2}=0$, is by definition the condition that the vector bundle is holomorphic (i.e., that its transition functions are holomorphic functions over the base $X_{3}$ ). The consequences of the condition $g^{a \bar{b}} F_{a \bar{b}}=0$ are not so easy to state, however; solving this partial differential equation has historically posed a significant challenge to the construction of supersymmetric heterotic vacua, since the background Ricci-flat Calabi-Yau metric $g^{a \bar{b}}$ and the field strength $F$ associated to the bundle $V$ are not known analytically except in very special cases. ${ }^{12}$ Thanks, however, to the powerful Donaldson-Uhlenbeck-Yau theorem [62-64], it is possible to translate this problem in differential geometry into one in algebraic geometry. According to the DUY theorem, a holomorphic bundle $V$ admits a connection $A$ that solves (5.3) if and only if $V$ is slope poly-stable. A bundle $V$ is defined to be slope stable with respect to a given Kähler form $\omega \in H^{1,1}\left(X_{3}\right)$ if for all sub-sheaves $\mathcal{F} \subset V$, with $0<r k(\mathcal{F})<r k(V)$,

$$
\mu(\mathcal{F})<\mu(V)
$$

where for any sheaf,

$$
\mu(\mathcal{F})=\frac{1}{r k(\mathcal{F})} \int_{X_{3}} c_{1}(\mathcal{F}) \wedge \omega \wedge \omega
$$

A bundle is called semi-stable ${ }^{13}$ if $\mu(\mathcal{F}) \leq \mu(V)$ for all sub-sheaves, and "poly-stable" if $V=\bigoplus_{i} V_{i}$ with $V_{i}$ stable and $\mu(V)=\mu\left(V_{i}\right) \forall i$. Regardless of the structure group $H$ of $V$, vector bundles describing a good heterotic vacuum must be holomorphic, slope poly-stable and satisfy

$$
\mu(V)=0
$$

for the physical Kähler form $\omega$.

It is poly-stable bundles that we must consider in the context of $\mathcal{N}=14 \mathrm{D}$ heterotic Calabi-Yau vacua, and although the study of such bundles and their moduli spaces is a rich and ongoing subject in algebraic geometry, at present very little is known in general about how to fully classify and enumerate the moduli space of stable bundles (sheaves) on CalabiYau threefolds. ${ }^{14}$ One of our goals in this work is to try to use heterotic/F-theory duality to understand as much as possible about which stable bundles can exist on Calabi-Yau threefolds and what properties characterize the associated heterotic effective theories.

\footnotetext{
${ }^{12}$ For recent progress in solving these equations via numeric approximations, see for example [92-94].

${ }^{13}$ Note that all poly-stable bundles are automatically semi-stable, but the converse does not hold.

${ }^{14}$ See $[78,95,96]$ for some recent results in the math/physics literature on bundle moduli spaces on Calabi-Yau threefolds in examples with special topology.
} 


\subsection{The Bogomolov bound}

There are a number of constraints that slope-stability places on the topology of a holomorphic vector bundle. One of the most important of these is the so-called "Bogomolov bound" (see [97] for a review), which states that if a rank $N$ bundle $V$ is slope (poly-) stable with respect to a choice of Kähler form $\omega=t^{k} \omega_{k}$ (with $k=1, \ldots h^{1,1}\left(X_{3}\right)$ ) on the CY 3-fold $X_{3}$, then

$$
\int_{X_{3}}\left(2 N c_{2}(V)-(N-1) c_{1}^{2}(V)\right) \wedge \omega \geq 0
$$

For simplicity, let us consider first the case of vector bundles with $c_{1}(V)=0$, in which case the Bogomolov bound reduces to $\int_{X_{3}} c_{2}(V) \wedge \omega \geq 0$.

Thus far our discussion of the consistency conditions on heterotic vacua has been general. We restrict our attention now to those threefolds that can give rise to F-theory duals, namely smooth, elliptically fibered Calabi-Yau threefolds, $\pi: X_{3} \rightarrow B_{2}$ (with section). More specifically, we restrict our consideration to the case in which there is a single section (i.e., the Mordell-Weil group of sections is trivial and $h^{1,1}\left(X_{3}\right)=1+h^{1,1}\left(B_{2}\right)$ ) and the manifold can be put in Weierstrass form as

$$
\hat{Y}^{2}=\hat{X}^{3}+f(u) \hat{X} \hat{Z}^{4}+g(u) \hat{Z}^{6}
$$

where $\{\hat{X}, \hat{Y}, \hat{Z}\}$ are coordinates on the elliptic fiber (described as a degree six hypersurface in $\mathbb{P}_{231}$ ) and $\{u\}$ are coordinates on the base $B_{2}$.

Let us consider the consequences of the Bogomolov bound for a bundle over an $X_{3}$ defined as above. Recalling the geometric identities in appendix A, as in (A.9) we can expand the second Chern class of the bundle as

$$
c_{2}(V)=\pi^{*}(\eta) \wedge \omega_{0}+\pi^{*}(\zeta)
$$

where $\pi^{*}(\eta)$ and $\pi^{*}(\zeta)$ are pullbacks of, respectively, $\{1,1\}$ and $\{2,2\}$ forms on the base $B_{2}$. (Note that in other sections we often use the notation e.g. $\eta$ both for the form on $B_{2}$ and for the pullback form - technically $\pi^{*}(\eta)-$ on $\mathcal{B}_{3}$. Which form is used should be apparent in any given equation from context.) Expanding the Kähler form $\omega$ in the explicit basis of appendix section $\mathrm{A}$, we have

$$
\omega=a \omega_{0}+\pi^{*}\left(\omega^{\text {base }}\right)
$$

where $a$ is a constant and $\omega_{\text {base }}=b^{\alpha} \omega_{\alpha}\left(\alpha=1, \ldots h^{1,1}\left(B_{2}\right)\right)$ is an ample divisor on $B_{2}$. Without loss of generality, we can scale the Kähler form to set $a=1$

$$
\omega=\omega_{0}+M \pi^{*}\left(\omega^{\text {base }}\right)
$$

for some constant $M$. Substituting this form for the Kähler moduli into the Bogomolov bound, the constraint becomes

$$
\begin{aligned}
& \int_{X}\left(\pi^{*}(\eta) \wedge \omega_{0}+\pi^{*}(\zeta)\right) \wedge\left(\omega_{0}+M \pi^{*}\left(\omega_{\text {base }}\right)\right) \\
& =M \omega_{0} \wedge \pi^{*}\left(\eta \wedge \omega_{\text {base }}\right)+\left(\omega_{0} \wedge \pi^{*}(\zeta)+\pi^{*}(\eta) \wedge \omega_{0} \wedge \omega_{0}\right) \geq 0
\end{aligned}
$$


Moreover, we recall that by the triple intersection numbers in (A.5),

$$
\int_{X_{3}} \omega_{0} \wedge \pi^{*}\left(\eta \wedge \omega_{\text {base }}\right)=\eta \cdot \omega_{\text {base }},
$$

and $\pi^{*}(\eta) \wedge \omega_{0} \wedge \omega_{0}=\eta \cdot K_{B_{2}}$. Thus, the Bogomolov bound becomes the condition

$$
\int_{X_{3}} c_{2}(V) \wedge \omega=M \eta \cdot \omega_{\text {base }}+\zeta+\eta \cdot K_{B_{2}} \geq 0
$$

(with $\zeta$ viewed now as the coefficient of the $\{2,2\}$-form on $B_{2}$ ). Note that in (5.13) and (5.14) while the l.h.s. is computed by integrating over the threefold $X_{3}$, the r.h.s. is computed in terms of intersection products on the base $B_{2}$.

In general, to extract consistency conditions on $\eta$ from the Bogomolov bound, the condition in (5.14) must be examined in a case-by-case manner. That is, given a choice of $\zeta$ and a Kähler form in (5.11), it is possible to derive consistency conditions on $\eta$ associated to the underlying bundle being slope-stable (though once again it is important to recall that the Bogomolov bound is necessary but not sufficient for the stability of $V$ ).

There is one limit, however, that is of particular interest in heterotic/F-theory duality. In order to take the stable degeneration limit of section 3, it is necessary that we evaluate this expression with not just any Kähler form, but one chosen in the appropriate "Adiabatic limit" [19] in which the volume of the elliptic fiber is small compared to that of the base (and the volume of $X_{3}$, as given in (A.6), is large). This limit is achieved by taking $M \gg 1$ in (5.14). For $M$ sufficiently large, it is clear that the dominant constraint from the Bogomolov bound in (5.14) is that $\eta$ has positive intersection with the Kähler form of the base $B_{2}$. In the adiabatic limit, it is impossible for the Bogomolov bound to be satisfied unless

$$
\eta \cdot \omega_{\text {base }} \geq 0
$$

If this is taken to hold for any ample Kähler form $\omega_{\text {base }}$ of the base then, by definition, this is simply the condition that $\eta$ is an effective divisor in $B_{2}$.

\subsection{Matter spectra in heterotic theories}

As described above, the presence of a vector bundle $V_{i}$ with structure group $H_{i}$ on the Calabi-Yau threefold breaks $E_{8}$ to $G_{i}$, the commutant of $H_{i}$ inside of $E_{8}$. All matter in the low-energy effective theory arises under dimensional reduction from components of the 10-dimensional gauge field, that is from the decomposition of the 248-dimensional representation of $E_{8}$ under the direct product $G_{i} \times H_{i}$ (see [1] for a standard review):

$$
\mathbf{2 4 8} \rightarrow(\operatorname{Ad}(G), 1)+\bigoplus_{A}\left(R_{A}, r_{A}\right)
$$

where $\operatorname{Ad}(G)$ represents the adjoint representation of $G$ and $\left\{\left(R_{A}, r_{A}\right)\right\}$ denotes a set of representations of $G \times H$. For example, the presence of a bundle with $H=\mathrm{SU}(3)$ over $X_{3}$ breaks one $E_{8}$ factor down to $G=E_{6}$ and the possible states in the theory are determined by the decomposition

$$
248 \rightarrow(78,1)+(27,3)+(\overline{27}, \overline{3})+(\mathbf{1}, 8)
$$




\begin{tabular}{|c|c|}
\hline Field & Multiplicity \\
\hline $\mathbf{2 7}$ & $h^{1}(X, V)$ \\
\hline$\overline{\mathbf{2 7}}$ & $h^{1}\left(X, V^{\vee}\right)$ \\
\hline $\mathbf{1}$ & $h^{1}\left(X, \operatorname{End}_{0}(V)\right)$ \\
\hline
\end{tabular}

Table 2. Matter multiplicities for the $E_{6}$ example.

That is, in addition to the adjoint-valued $E_{6}$ gauge boson, the low energy theory can contain charged matter in the $\mathbf{2 7}$ and $\overline{\mathbf{2 7}}$ representations, as well as $E_{6}$-singlet fields.

While the decomposition above is sufficient to determine the type of matter in the 4dimensional $E_{6}$ theory, to find the multiplicity of these massless scalar fields it is necessary to count the number of bundle-valued 1-forms on the Calabi-Yau manifold. More precisely, under dimensional reduction, the zero-modes of the 4-dimensional theory are determined by the dimensions of vector bundle-valued cohomology groups (in the representation $r_{A}$ ) over the Calabi-Yau threefold, such as

$$
H^{1}\left(X_{3}, V\right), H^{1}\left(X_{3}, \wedge^{2} V\right), H^{1}\left(X, \operatorname{End}_{0}(V)\right), \text { etc. } \ldots
$$

For the $E_{6}$ example above this leads to the bundle-valued cohomology groups shown in table 2 where $V$ is the rank 3 vector bundle valued in the fundamental of SU(3) (and hence $V^{\vee}$ is associated to the $\overline{\mathbf{3}}$ and $\operatorname{End}_{0}(V)$ to the $\left.\mathbf{8}\right)$.

The chiral index ${ }^{15}$ of the $\mathcal{N}=1$ theory is determined by the Atiyah-Singer index theorem [98] as the alternating sum

$$
\operatorname{Ind}(V)=h^{0}(X, V)-h^{1}(X, V)+h^{2}(X, V)-h^{3}(X, V) .
$$

But by slope stability of the bundle $V$ and the condition $c_{1}(V)=0$,

$$
H^{0}\left(X_{3}, V\right)=H^{0}\left(X_{3}, V^{\vee}\right)=0
$$

(as well as the induced representations; i.e., $H^{0}\left(X, \wedge^{n} V\right)=0$, with $n<r k(V)$, etc). Finally, it should be recalled that by Serre duality [98], $h^{m}(X, V)=h^{3-m}\left(X, V^{\vee}\right)$ and as a result, the chiral index can be expressed simply as the difference,

$$
\operatorname{Ind}(V)=-h^{1}(X, V)+h^{1}\left(X, V^{\vee}\right) .
$$

In the case that $c_{1}(V)=0$ this is further given by $\operatorname{Ind}(V)=\mathrm{Ch}_{3}(V)=\frac{1}{2} c_{3}(V)$, the third Chern character. Finally, it should be noted that the index given in (5.21) is written in terms of the vector bundle associated to the fundamental representation of the underlying principal $H$-bundle. The chiral asymmetry in all other representations (i.e., for the induced vector bundles, $\left.V^{\vee}, \wedge^{m} V, S^{p} V, \ldots\right)$ is fact determined by the index of the fundamental representation given above (see [99] for further details). In heterotic theories, the exact

\footnotetext{
${ }^{15}$ Physically, the chiral index in a heterotic compactification counts the number of generations minus the number of anti-generations of chiral particles. For example, the number of $\mathbf{2 7}$ multiplets minus the number of $\overline{\mathbf{2 7}}$ 's in the $E_{6}$ theory given above, or the number of families in a heterotic Standard Model.
} 
massless matter spectrum is frequently easier to compute than in F-theory, and in this work we use the simple structure of heterotic matter to extract useful information about the spectrum and chiral index arising from F-theory compactifications on Calabi-Yau fourfolds.

For heterotic theories defined over elliptically fibered Calabi-Yau threefolds, the bundle-valued cohomology groups defined above have a simple decomposition in terms of the base/fiber geometry. Using the techniques of Leray spectral sequences, there exists a decomposition

$$
H^{m}(X, V)=\bigoplus_{p+r=m} H^{r}\left(B_{2}, R^{p} \pi_{*}(V)\right)
$$

where $R^{p} \pi_{*}(V)$ is the $p$-th derived push forward [98] of $V$. On any open set $\mathcal{U}, R^{p} \pi_{*}(V)$ can locally be represented on $B_{2}$ by the pre-sheaf

$$
\mathcal{U} \rightarrow H^{p}\left(\pi^{-1}(\mathcal{U}), V\right)
$$

This formalism allows for a very precise notion of "localized" matter in the heterotic theory (supported over loci in the base $B_{2}$ ) which (in the case of simply-laced $G$ ) can be matched exactly to the localized matter associated to 7-brane intersections in the dual F-theory geometry. We explore this localized matter and the chiral index further in section 7.8 and in the context of the spectral cover construction of vector bundles below.

\subsection{The spectral cover construction}

Thus far our discussion of heterotic compactifications and constraints has been completely general, and throughout this work we attempt as far as possible to keep our study of the holomorphic vector bundles $\left(V_{1}, V_{2}\right)$ independent of any particular method of bundle construction. It will, however, be useful in certain examples to appeal to one method of constructing vector bundles on elliptically fibered manifolds in which heterotic/F-theory duality is particularly well understood [19, 42]. This is the well-known "spectral cover" construction [39, 41].

\subsubsection{Spectral covers}

The spectral cover construction can be used to build rank $N$ bundles, $V \rightarrow X_{3}$, with structure group $\mathrm{SU}(N)$ or $\mathrm{Sp}(2 N)$. Moreover, for some bundles with these structure groups, which are slope-stable in the adiabatic region described in the previous section, the FourierMukai transform ${ }^{16}$ [40] provides a 1-1 map (in fact a full functor on the category of coherent sheaves) from $V$ to a pair $\left(\mathcal{S}, L_{\mathcal{S}}\right)$ where $\mathcal{S}$ is a divisor in $X_{3}$ that is an $N$-fold cover of the base $B_{2}$ and $L_{\mathcal{S}}$ is a rank- 1 sheaf on $\mathcal{S}$. The class of $\mathcal{S}$ is given by

$$
[\mathcal{S}]=N[\sigma]+\pi^{*}(\eta)
$$

where $\sigma$ is the zero section of $\pi: X_{3} \rightarrow B_{2}$ and $\eta$ is defined as in (5.9). As in (5.8), let $\hat{X}, \hat{Y}, \hat{Z}$ be the coordinates of the elliptic fiber (where $\hat{Z}=0$ defines the section $\sigma$ ). Then

\footnotetext{
${ }^{16}$ The precise conditions for stability and consistency of spectral cover bundles will be discussed further in the next section.
} 
in the case that the structure group of $V$ is $\mathrm{SU}(N)$, the spectral cover $\mathcal{S}$ can be represented as the zero set of the polynomial

$$
s=a_{0} \hat{Z}^{N}+a_{2} \hat{X} \hat{Z}^{N-2}+a_{3} \hat{Y} \hat{Z}^{N-3}+\ldots
$$

ending in $a_{N} \hat{X}^{\frac{N}{2}}$ for $N$ even and $a_{N} \hat{X}^{\frac{N-3}{2}} \hat{Y}$ for $N$ odd [19] (see [19] also for the analogous construction for $\operatorname{Sp}(2 N)$ structure group). The coefficients $a_{j}$ are sections of line bundles over the base $B_{2}$

$$
a_{j} \in H^{0}\left(B_{2}, K_{B_{2}}^{\otimes j} \otimes \mathcal{O}(\eta)\right)=H^{0}\left(B_{2}, \mathcal{O}\left(\eta+j K_{2}\right)\right),
$$

which can locally be described as polynomial functions with appropriate degrees. Note that in the duality to F-theory, the coefficients $a_{j}$ play a dual role as coefficients $f_{k}, g_{k}$ in the F-theory Weierstrass model, providing a direct map between the moduli on the two sides of the duality. In order for the spectral cover to be an actual algebraic surface in $X_{3}$ it is necessary that $\mathcal{S}$ be an effective class in $H_{4}\left(X_{3}, \mathbb{Z}\right)$. It is straightforward to show $[19,39,100]$ that this is true if and only if $\eta$ is an effective class in $B_{2}$. This can be seen by noting that $\eta$ must be effective for $a_{0}$ to be nonvanishing, and since $-K_{2}$ is effective no other coefficient $a_{j}$ can be nonvanishing if $\eta$ is not effective. In view of the Bogomolov condition in the previous section, it is clear that spectral cover bundles are built to be slope-stable in the adiabatic region of Kähler moduli space. ${ }^{17}$

There is a further condition that must be imposed in order for the spectral cover bundle $V$ to be slope stable. By construction, irreducible spectral covers are stable in the adiabatic region given above $[19,39]$. However, the condition that the cover is irreducible places another condition on $\eta$. It can be argued that $\mathcal{S}$ is irreducible ${ }^{18}$ if $\eta$ is base-point free (i.e., has no base locus in a decomposition of the type (4.1)) and $\eta-N c_{1}\left(B_{2}\right)$ is effective (see [102] for example). The condition of base point free-ness will be explored in further detail for surfaces in section 6.4, but for now it should simply be noted that this condition guarantees that there exist irreducible curves in the class $[\eta]$.

To fully determine the bundle $V$ and its topology after Fourier-Mukai transform, it is necessary to specify not only the class $\eta$ in (5.24), but also the rank-1 sheaf $L_{\mathcal{S}}$ above. The condition that $c_{1}(V)=0$ fixes the first Chern class of $L_{\mathcal{S}}$ to be [19]

$$
c_{1}\left(L_{\mathcal{S}}\right)=N\left(\frac{1}{2}+\lambda\right) \sigma+\left(\frac{1}{2}-\lambda\right) \pi_{S}^{*} \eta+\left(\frac{1}{2}+N \lambda\right) \pi_{S}^{*} c_{1}\left(B_{2}\right)
$$

\footnotetext{
${ }^{17}$ More precisely, it is known [100] that for a spectral cover bundle there exists some value $M_{0}$ such that for $M \gg M_{0}$ in (5.11), the bundle associated to that spectral data is slope stable for the given region of Kähler moduli space. For Kähler twofolds the proof of stability is constructive and yields an explicit value of $M_{0}$ defining the stable region of Kähler moduli space. For Calabi-Yau threefolds however, the arguments are not constructive and we are restricted to considering the limit $M \gg 1[19,100]$.

${ }^{18}$ Note that if $\mathcal{S}$ is irreducible as an algebraic curve in $X_{3}$, the associated vector bundle under FourierMukai transform will be indecomposable (i.e. not a direct sum $V_{1} \oplus V_{2} \oplus \ldots$ ). However, the converse does not hold. Some reducible spectral covers can still correspond to indecomposable vector bundles. See $[71,101]$ for examples.
} 
where $\pi_{\mathcal{S}}: \mathcal{S} \rightarrow B_{2}$, the bundle $V$ has structure group $H=\mathrm{SU}(N)$, and the parameter $\lambda$ is either integer or half-integer depending on $N$ :

$$
\lambda= \begin{cases}m+\frac{1}{2}, & \text { if } N \text { is odd } \\ m, & \text { if } N \text { is even }\end{cases}
$$

where $m \in \mathbb{Z}$. This condition arises from the fact that $c_{1}\left(L_{\mathcal{S}}\right)$ must be an integral class in $H^{1,1}(\mathcal{S}, \mathbb{Z})$. When $N$ is even it is clear that this integrality condition imposes

$$
\eta \equiv c_{1}\left(B_{2}\right) \bmod 2
$$

where "mod 2" indicates that $\eta$ and $c_{1}\left(B_{2}\right)$ differ only by an even element of $H^{2}\left(B_{2}, \mathbb{Z}\right)$. The relation (5.27) holds when the cohomology of $\mathcal{S}$ is spanned by the class $\sigma$ and the pullback of the cohomology in the base. While this is expected to be true generically, there can be situations in which $\mathcal{S}$ has a larger Picard group (i.e. more independent divisor classes). Examples where this increase in $h^{1,1}(\mathcal{S})$ may occur include Noether-Lefschetz loci in the complex structure of $\mathcal{S}$ and degenerate (singular) spectral covers (see [103] for some generalizations of (5.27) for such examples). In these more general situations, the constraint (5.29) may not hold. We will see the need for such interesting possibilities (and their F-theory duals) in later sections.

Finally, with this data in hand it is possible to extract the full topology of $V$, including the chiral index, $\operatorname{Ind}(V)=-h^{1}\left(X_{3}, V\right)+h^{1}\left(X_{3}, V^{\vee}\right)$. The Chern classes of a spectral cover bundle $V$, specified by $\eta$ and the integers $N$ and $\lambda$, are [19, 39, 45, 103]

$$
\begin{aligned}
& c_{1}(V)=0 \\
& c_{2}(V)=\eta \wedge \sigma-\frac{N^{3}-N}{24} c_{1}\left(B_{2}\right)^{2}+\frac{N}{2}\left(\lambda^{2}-\frac{1}{4}\right) \eta \wedge\left(\eta-N c_{1}\left(B_{2}\right)\right) \\
& c_{3}(V)=2 \lambda \sigma \wedge \eta \wedge\left(\eta-N c_{1}\left(B_{2}\right)\right)=2 \lambda \eta \cdot\left(\eta-N c_{1}\left(B_{2}\right)\right)
\end{aligned}
$$

Note that since $c_{1}(V)=0, \operatorname{Ind}(V)=\operatorname{ch}_{3}(V)=\frac{1}{2} c_{3}(V)$.

The essential heterotic constraints on a bundle constructed via spectral covers can be simply encapsulated by the $\{1,1\}$ form $\eta$. For a bundle $V_{i}$, if $\eta_{i}$ and $\eta_{i}-N c_{1}\left(B_{2}\right)$ are effective, $\eta_{i}$ is base-point free, and an $L_{\mathcal{S}}$ is chosen subject to (5.27), (5.28) and (5.29), a spectral cover bundle is guaranteed to exist and to be slope stable (for a region in Kähler moduli space in which heterotic/F-theory duality is well understood). In section 6 we explore the way that some of these same constraints appear in the dual fourfold geometry, giving information about when these constraints must be true based on topological data independent of a specific method of bundle construction.

\subsubsection{Localized matter and spectral covers}

In $\mathrm{SU}(N)$ spectral covers, at least some of the zero-modes of the theory have a simple realization in terms of the geometry of the spectral cover $\mathcal{S} \subset X_{3}$. By the Leray spectral sequence arguments outlined above, it can be shown that the matter in the theory determined by $H^{1}(X, V)$ is localized at the intersections $\mathcal{S} \cap \sigma$ in $B_{2}$ (see section (6.2) of [19] 
for a review). For example, for an $\mathrm{SU}(2)$ spectral cover of the form

$$
a_{0} \hat{Z}^{2}+a_{2} \hat{X}=0
$$

$\mathcal{S}$ intersects the zero-section $\sigma$ at the zero-locus of the section $a_{2}(u) \in H^{0}\left(B_{2}, K_{2}^{\otimes 2} \otimes \mathcal{O}(\eta)\right)$ over $B_{2}$. This localized matter appears as the $\mathbf{5 6}$ multiplets of the 4 -dimensional $E_{7}$ theory. The exact multiplicity of these fields can be found by a Leray calculation to determine the exact zero-mode spectrum along the curve $a_{2}=0$ in $B_{2} \cdot{ }^{19}$

More generally, given an $\mathrm{SU}(N)$ spectral cover of the form shown in (5.25), the localized matter counted by $H^{1}(X, V)$ (i.e., the matter valued in the fundamental representation of $V$ ) will be controlled by the zeros of $a_{N}(u) \in H^{0}\left(B_{2}, K_{2}^{\otimes N} \otimes \mathcal{O}(\eta)\right)$. Note that this matter need not be chiral, and this is of course not the full matter spectrum of the low-energy $4 \mathrm{D}$ theory - for that other representations appearing in the decomposition of the $\mathbf{2 4 8}$ of $E_{8}$ in (5.16), such as $H^{1}\left(X, \wedge^{2} V\right), H^{1}\left(X, \operatorname{End}_{0}(V)\right)$ etc., must be considered. For these, we must consider not just the $N$-sheeted spectral cover associated to the fundamental representation of the $\mathrm{SU}(N)$ bundle, but other curves associated to other induced vector bundles (such as $\wedge^{k} V, S^{p} V$, etc) as well. At present, it is not known how to construct all such associated spectral covers in full generality (see [20, 104] for some progress in this direction), though in the case of some of these representations there will likewise be a notion of localized matter [20].

Finally, it is worth noting that although the presence of "matter curves" in the class $\left[a_{N}=0\right]$ in $B_{2}$ indicates the presence of charged matter in the $4 \mathrm{D}$ theory, the exact matter spectrum with multiplicities cannot be determined without fully specifying all the data of the bundle, including detailed properties of a particular $V$ (not just its topology) and of course, the third Chern class (5.21). With this data in hand, the restriction of $V$ to both the fiber and base implicit in (5.22) and (5.23) can be explicitly calculated. From the perspective of F-theory, we expect this further data to be necessary, since it is known that the exact (chiral) matter spectrum depends crucially not just on the fourfold geometry, but also a choice of $G$-flux. We will return to the issue of chiral matter and heterotic/F-theory duality again in section 7.8 .

\subsubsection{Limitations of spectral covers}

Despite the fact that it constitutes one of the most studied and best understood corners of the dual heterotic/F-theory landscape, the spectral cover construction is far from general and care must be taken in generalizing results derived in this context to the full vector bundle moduli space or the generic dual fourfold geometries. As pointed out above, the spectral cover construction is valid only for special structure groups (i.e., $H=\mathrm{SU}(N)$ or $\mathrm{Sp}(2 N))$ and perhaps more importantly, its applicability is limited even in these settings. That is, not all consistent $\mathrm{SU}(N)$ or $\mathrm{Sp}(2 N)$ bundles arising in heterotic compactifications can be represented by well-behaved spectral covers.

\footnotetext{
${ }^{19}$ Note that in the dual F-theory geometry matter curves appear in the shared base $B_{2}$ in exactly the same way. In the notation of (4.5)-(4.6), the dual Weierstrass model to (5.33) is $y^{2}=x^{3}+\left(a_{2} z^{3}+\ldots\right) x+\left(a_{0} z^{5}+\ldots\right)$ and on the vanishing locus $a_{2}=0$ there is an enhancement of $E_{7} \rightarrow E_{8}$ as expected.
} 
Since this has an impact on the heterotic/F-theory comparisons undertaken in this work, it is worth briefly reviewing some of these constraints here. The Fourier-Mukai transform is a well-behaved functor on the moduli space of sheaves, subject to the following conditions: that the restriction $\left.V\right|_{E_{p}}$ of the bundle to each elliptic fiber $E_{p}$ is

\section{Semistable}

2. Regular (i.e., that the restricted rank $N$ bundle on the elliptic curve has an automorphism group of the minimum possible dimension: $\left.\operatorname{dim}\left(\operatorname{Aut}\left(\left.V\right|_{E_{p}}\right)\right)=N\right)$

For the first of these, the semi-stability of $V$ is defined with respect to the restricted slope in (5.5). For indecomposable stable bundles $V$ this will be true for generic fibers when the Kähler class is chosen to be

$$
\omega=\omega_{0}+M \omega_{\alpha} \quad, \quad M \gg 1
$$

where, as above, for threefolds the stability proof is based on $M>M_{0}$ for some unknown $M_{0}$ and is not constructive. The condition above is sufficient to guarantee that a stable region for $V$ exists, compatible with the large volume, weakly-coupled limit that we require for heterotic/F-theory dual pairs. It is important to note, however, that this limitation of moduli space is certainly not necessary for the consistency of heterotic theories $[105,106]$. This point is explored further in appendix B.

The second condition of "regularity" appearing above was introduced by Atiyah in his classification of semi-stable sheaves on elliptic curves [107]. In particle, $V$ is called "regular" if when it is restricted to every elliptic fiber it decomposes into a poly-stable sum of line bundles (i.e., by the divisor-line bundle correspondence, a set of points on $E_{i}$ summing to zero) rather than a non-trivial extension (for example, Atiyah's $I_{2}$ bundle of the form $\left.0 \rightarrow \mathcal{O}_{E_{i}} \rightarrow I_{2} \rightarrow \mathcal{O}_{E_{i}} \rightarrow 0\right)$. Unlike the previous one, this condition cannot be stated as a simple global restriction on the heterotic moduli space and is harder to characterize for generic bundles. Indeed, it is significant that many good heterotic bundles will fail the regularity criterion. For instance, it is known that most bundles described via the monad construction $[108,109]$ are not regular [110]. In fact, there are indications that this criteria can sometimes be consistently violated in the context of perturbative heterotic/F-theory dual geometry (including in such well-known examples [71] as the heterotic "Standard Embedding" in which $V=T X_{3}$ ). See [101] for some recent results on degenerate spectral covers and dual F-theory geometry. The regularity condition will not be explored here in detail, but it may be relevant in explaining some of the unsolved questions regarding geometric constraints arising in dual heterotic/F-theory pairs, including exotic G-flux (see section 7.9) and the role of quantization conditions like (5.28) and (5.29).

\section{Equivalence of constraints}

We now consider the relation between constraints on F-theory geometry and the constraints on bundle constructions on the heterotic side. 


\subsection{Effective condition on $\eta_{i}$}

We begin by showing that the F-theory condition that $f, g$ do not vanish to degrees 4,6 on either $\Sigma_{-}$or $\Sigma_{+}$is equivalent to the heterotic condition that $\eta_{1}$ and $\eta_{2}$ are effective. This follows directly from the analysis of section 4.1.5, where it was shown that $-6 K_{2} \pm T$ must be effective in any F-theory geometry where $f, g$ are well-behaved on $\Sigma_{ \pm}$. With the identification

$$
\eta_{ \pm}=\eta_{1,2}=-6 K_{2} \pm T
$$

it follows that the condition that $\eta_{ \pm}$are effective is a necessary condition for the existence of a good F-theory geometry. This is thus a necessary condition for the existence of a good heterotic dual. This matches with what is known of the heterotic theory, where this bound is necessary in order to satisfy the Bogomolov bound in the adiabatic limit of the stable degeneration limit. The fact that this bound is necessary for any good F-theory geometry shows that this bound on the heterotic side must be more general and applies to any bundle construction, irrespective of of the stable degeneration limit.

In fact, from the F-theory side the constraint is significantly stronger. The constraint on the F-theory side states that either $\eta_{-}=0$, or $-4 K_{2}-T=\eta_{-}-2 c_{1}$ must be effective. This constraint, and the analogous constraint for $\eta_{+}$must be necessary conditions on the heterotic side for the existence of any smooth bundle with the specified components of $c_{2}$.

\subsection{Effective constraint and gauge groups}

In [38] the effective constraint on $\eta$ for a bundle to exist on the heterotic side was generalized to situations where the $4 \mathrm{D}$ gauge group can be seen to be restricted in specific ways on the F-theory side. These constraints can be readily attained by a generalization of the analysis in the previous section. For example, if we consider a divisor $\Sigma_{-}$that carries a gauge group no larger than $E_{7}$, then $-4 K_{2}-T$ must be effective for $f$ to have a term of degree $\leq 3$, so $\eta=6 c_{1}-T \geq 2 c_{1}$, where by $A \geq B$ we mean that $A-B$ is effective. This corresponds on the heterotic side to a constraint on $\eta$ for bundles with structure group $H=\mathrm{SU}(2)$, so we conclude that $\eta \geq 2 c_{1}$ is a necessary condition for the existence of a bundle with structure algebra $\mathfrak{s u}_{2}$ (or greater). This matches with the result found in [38]. Similarly, bundles with structure $\mathfrak{s u}_{3}$ or $\mathfrak{g}_{2}$ correspond to gauge algebras $\mathcal{G}=\mathfrak{e}_{6}, \mathfrak{f}_{4}$, which have $g_{4}$ or a lower term in $g$ or $f$ vanishing, so $-6 K_{2}-2 t=2 \eta-6 c_{1}=2\left(\eta-3 c_{1}\right)$ is effective. The results for these and the other minimal gauge group types are shown in table 3, again in agreement with [38]. Note that these conditions for bundles with structure group $\mathrm{SU}(2), \mathrm{SU}(3)$ precisely agree with the condition that $\eta-N c_{1}\left(B_{2}\right)$ is effective that was needed in section 5.3, showing that this constraint is more general and independent of bundle construction. In the case where $G$ is trivial and the structure group on the heterotic side is $E_{8}$, it is only necessary that $-6 K_{2}-5 t=5\left(\eta-24 c_{1} / 5\right)$ be effective (corresponding to a nontrivial $\left.g_{1}\right)$. For $\mathrm{SU}(N)$ groups with $N>3$, on the other hand, $f_{0}$ and $g_{0}$ must be nonvanishing so $-6 K_{2}-6 t=6\left(\eta-5 c_{1}\right)$ must be effective and $\eta \geq 5 c_{1}$. 


\begin{tabular}{|ccc|}
\hline $\mathcal{G}$ & $\mathcal{H}$ & bound \\
\hline $\mathfrak{e}_{8}$ & 1 & $\eta=0$ \\
$\mathfrak{e}_{7}$ & $\mathfrak{s u}_{2}$ & $\eta \geq 2 c_{1}$ \\
$\mathfrak{e}_{6}, \mathfrak{f}_{4}$ & $\mathfrak{s u}_{3}, \mathfrak{g}_{2}$ & $\eta \geq 3 c_{1}$ \\
$\mathfrak{s o}_{8}, \mathfrak{g}_{2}$ & $\mathfrak{s o}_{8}, \mathfrak{f}_{4}$ & $\eta \geq 4 c_{1}$ \\
$\mathfrak{s u}_{3}$ & $\mathfrak{e}_{6}$ & $\eta \geq \frac{9}{2} c_{1}$ \\
$\mathfrak{s u}_{2}$ & $\mathfrak{e}_{7}$ & $\eta \geq \frac{14}{3} c_{1}$ \\
1 & $\mathfrak{e}_{8}$ & $\eta \geq \frac{24}{5} c_{1}$ \\
\hline
\end{tabular}

Table 3. Constraints on $\eta$ for certain structure groups $H$ of heterotic bundles, identified from F-theory bounds on $\eta$ for a given $4 \mathrm{D}$ gauge group $G ; \eta \geq a c_{1}$ means that $\eta-a c_{1}\left(B_{2}\right)$ is an effective divisor on $B_{2}$. For example, a heterotic bundle with structure group $\mathrm{SU}(2)$ is only possible when $\eta-2 c_{1}$ is effective. These results match those found in [38].

\subsection{F-theory constraints and $\mathrm{SO}(32)$ models}

As discussed in section 3 , for any given base $B_{2}$ there is a unique twist $T=2 c_{1}\left(B_{2}\right)$ so that F-theory on the resulting $\mathcal{B}_{3}$ is dual to the $\mathrm{SO}(32)$ heterotic theory on a generic elliptically fibered Calabi-Yau threefold over $B_{2}$. In this case the F-theory conditions are that $f_{2}$ is a section of $\mathcal{O}\left(-4 K_{2}-2 T\right)=\mathcal{O}_{\mathcal{B}_{3}}$ and $g_{3}$ is a section of $\mathcal{O}\left(-6 K_{2}-3 T\right)=\mathcal{O}_{\mathcal{B}_{3}}$. Both of these are therefore simply complex numbers so that the cubic $x^{3}+f_{2} x+g_{3}$ has three complex roots and the resulting gauge algebra is $\mathfrak{s o}_{8}$. This matches the dual heterotic theory where a generic choice of bundle will break the full $\mathrm{SO}(32)$ (really Spin $(32) / Z_{2}$ ) down to $\mathrm{SO}(8)$, just as in the $6 \mathrm{D}$ case where the F-theory dual of heterotic on K3 is F-theory on an elliptically fibered Calabi-Yau threefold over the base $F_{4}$.

\subsection{Base-point free condition}

We now consider the conditions that the heterotic base-point free condition imposes on F-theory geometry. On the heterotic side, the constraint is that $\eta_{i}$ is base-point free on $B_{2}$. As discussed in section 4.1 , on a surface this simply means that there does not exist any effective divisor (curve) $D$ of negative self-intersection such that $\eta_{i} \cdot D<0$. In the F-theory picture this means that

$$
\left(-6 K_{2} \pm T\right) \cdot D \geq 0
$$

for all effective divisors $D$ in $B_{2}$. Let us examine the consequences of this condition for curves satisfying $D \cdot D=-n$ where $n=1,2$.

For a curve $D$ with $n=1$, we have $-K_{2} \cdot D=1$, since $\left(K_{2}+D\right) \cdot D=2 g-2=-2$ where $D$ is a rational curve, so (6.2) becomes

$$
6 \pm T \cdot D \geq 0
$$

This is automatically satisfied from (4.9). Thus, the base-point free condition imposes no additional conditions for F-theory bases associated with twists over curves of selfintersection -1 . In particular, this means that any F-theory base $\mathcal{B}_{3}$ formed as a $\mathbb{P}^{1}$ bundle 
over a del Pezzo base $B_{2}$ automatically gives rise to $\eta_{i}$ that satisfy the base-point free condition on the heterotic side, since $B_{2}$ contains no curves of self-intersection lower than -1 .

Now consider the case of a curve $D$ with self-intersection $-n=-2$. In this case, we have $-K_{2} \cdot D=0$, so (6.2) becomes

$$
\pm T \cdot D \geq 0
$$

This relation is only satisfied with both signs when $T \cdot D=0$. Thus, the base-point free condition will be violated whenever the twist over a -2 curve has $T \cdot D= \pm 1$. From (4.10), these are the only nonzero possibilities.

We can now analyze the consequences for the gauge group on the divisors $\Sigma_{ \pm}$when the base-point free condition is violated. This corresponds to the condition (C) analyzed in section 4.1.5. Assume that $D$ is a curve of self-intersection -2 with an associated twist having $T \cdot D=+1$. In this situation, $\left(-n K_{2}-(n-k) T\right) \cdot D=-(n-k)$. As described in section 4.1.3, this means that $g_{5}, g_{4}, f_{3}, f_{2}$ vanish on $D$ to degree at least $1, g_{3}, g_{2}, f_{1}, f_{0}$ vanish on $D$ to degree at least 2 , etc. An immediate consequence is that $f, g$ cannot vanish on $\Sigma_{-}$to degrees 3,5 or higher, or they would vanish on $\Sigma_{-} \cap D$ to degrees 4,6 . This means that there cannot be a generic $\mathfrak{e}_{7}$ or $\mathfrak{e}_{8}$ gauge group on $\Sigma_{-}$. Furthermore, if the degrees of vanishing are 3,4 then $g_{4}$ cannot be a perfect square, since if it was then it would vanish to degree two on $D$ and again $f, g$ would vanish to degrees 4,6 on $\Sigma_{-} \cap D$. As reviewed in section 3.1, this condition means that in the 3,4 vanishing case the generic gauge group must be $\mathfrak{f}_{4}$ and not $\mathfrak{e}_{6}$. Thus, in the non-base-point free cases where an $\mathrm{F}$ theory construction is possible, the generic gauge group cannot be $\mathfrak{e}_{6}, \mathfrak{e}_{7}$, or $\mathfrak{e}_{8}$. A similar consideration holds for the generic gauge group on $\Sigma_{+}$when $T \cdot D=-1$.

These conditions can be made more explicit in the toric context. Given a divisor $D_{i}$ with $\tilde{t}_{i}=T \cdot D_{i}=1$, we have a local set of rays in the toric fan as in (4.12)

$$
\begin{aligned}
s_{-} & =(0,0,1) \\
\tilde{w}_{i-1} & =(1,0,0) \\
\tilde{w}_{i} & =(0,-1,0) \\
\tilde{w}_{i+1} & =(-1,-2,1) .
\end{aligned}
$$

All monomials $m=(a, b, c) \in N^{*}$ in $\mathcal{F}, \mathcal{G}$ then satisfy the inequalities (4.14)-(4.16), as well as (4.18), which becomes $c \geq a+2 b-B$ for $B=4,6$. In addition, there must be at least one monomial $m$ that satisfies $(4.17), b-c>B$, for $B=4$ or 6 .

First, we can ask if it is possible to have a gauge group factor on $\Sigma_{-}$associated with a summand $\mathfrak{e}_{7}$ or $\mathfrak{e}_{8}$. From table 1 , this would mean that every monomial $m=(a, b, c)$ would have $c \geq-1$ for both $B=4$ and $B=6$. From figure 2 , it is clear that this is not possible, however. The only simultaneous solution in $(b, c)$ to $c<b-B, b \leq B, c \geq-1$ is $b=B, c=-1$, and this is ruled out for both values of $B$ by $c \geq a+2 b-B$ since $a \geq-B$. Thus, when the base-point free condition is violated through $\tilde{t}_{i}=+1$ over a -2 curve, the gauge algebra summand associated with $\Sigma_{-}$cannot be $\mathfrak{e}_{7}$ or $\mathfrak{e}_{8}$. A similar result follows for $\Sigma_{+}$when $\tilde{t}_{i}=-1$. 
Now let us consider the possible summands $\mathfrak{e}_{6}$ and $\mathfrak{f}_{4}$, both associated with vanishing degrees of 3,4 for $f, g$ on $\Sigma_{-}$. In this case the constraint for $B=6$ is $c \geq-2$. There is a simultaneous solution to the inequalities for this value of $c$, given by $m=(a, b, c)=$ $(-6,5,-2)$, again as depicted in figure 2 . So this combination of vanishing degrees can be realized. The distinction between $\mathfrak{e}_{6}$ and $\mathfrak{f}_{4}$ can be seen most easily from the leading term in the Weierstrass coefficient $g=g_{4} z^{4}+g_{5} z^{5}+\cdots$, in a local expansion in coordinates around the relevant divisor, which in this case is $\Sigma_{-}$. If $g_{4}$ is a perfect square, the gauge algebra is $\mathfrak{e}_{6}$, and otherwise it is $\mathfrak{f}_{4}$. Since the only allowed monomial at order $z^{4}$ is $g_{4}=y$, which is not a perfect square, the gauge algebra must always be $\mathfrak{f}_{4}$ when the base-point free condition fails on $\eta_{1}=6 c_{1}+T$ and the degree of vanishing of $f, g$ is 3,4 on $\Sigma_{-}$. The same result follows for $\Sigma_{+}$when $\tilde{t}_{i}=-1$.

The upshot of this analysis is that the only gauge algebras that are possible for the structure group of the bundle in the dual heterotic model when the base-point free condition is violated are the commutants of the possible gauge algebras of the $4 \mathrm{D}$ theory, namely $\mathfrak{e}_{8}, \mathfrak{e}_{7}, \mathfrak{e}_{6}, \mathfrak{f}_{4}, \mathfrak{s o}_{8}$, and $\mathfrak{g}_{2}$. This is in good agreement with what is known of heterotic/F-theory duality in these cases, since the spectral cover construction for $\operatorname{SU}(N)$ and $\operatorname{Sp}(N)$ structure group bundles is not possible when the base point free condition is violated. Furthermore, these results demonstrate that the base-point-free condition must be necessary for any heterotic bundle with structure group $\mathrm{SU}(N)$ or $\operatorname{Sp}(N)$, independent of the spectral cover construction.

For the gauge groups associated with the more general exceptional algebras, it is expected that other bundle constructions such as the more general "cameral cover" construction [40-42] will exist for the heterotic bundles (though explicit conditions on bundle topology are at present not as well understood in this context as they are in the case of spectral covers) and as a result, the base-point free condition is not necessarily a requirement for the construction of a sensible bundle. The analysis of this section suggests that all F-theory models on $\mathbb{P}^{1}$ bundles over $B_{2}$ 's that are generalized del Pezzo surfaces have well-defined heterotic duals, even when the base-point free condition is violated, though new tools may be needed for explicit construction of the appropriate bundles on the heterotic side.

\subsection{A note of caution: G-flux}

To close this section, we return briefly to a caveat mentioned in section section 1 regarding the results presented here that are based purely on F-theory geometry. In deriving the bounds on structure groups and $\eta$ in sections 3-6 we have ignored $G$-flux which must be taken into account for a full description of the F-theory physics and dual heterotic bundle moduli space. Some general aspects of G-flux in 4D F-theory models, and relevant references are given in section 7.8, section 7.9.

In some cases it may be possible for non-trivial G-flux (in the singular limit of the fourfold geometry) to change the apparent symmetry group that would be inferred from the Weierstrass equation. Although counterintuitive from the perspective of Abelian Gflux in a smooth M-theory limit, such symmetry-breaking by flux can be generic in the singular limit and is expected to occur in a wide range of $4 \mathrm{D}$ F-theory models. This has recently been explored in the context of local F-theory models as "T-branes" [111, 112] (or 
equivalently "gluing data" $[113,114])$ and in the global context in both 4- $[115,116]$ and 6-dimensional compactifications [101].

The basic mechanism by which G-flux can break an apparent symmetry appearing from the geometric F-theory analysis in terms of a Weierstrass model is most clear in the dual heterotic picture. As discussed in section 3.1, the geometry of the F-theory base $\mathcal{B}_{3}$ determines almost all of the topology of the corresponding bundle on the heterotic side. The components $\zeta_{i}$ in (3.2), however, are not determined by the 4 -fold geometry. On the heterotic side, non-trivial bundles with a second Chern class entirely in $\zeta_{i}$ can break the gauge group just as effectively as bundles with non-vanishing $\eta_{i}$ that have a clear dual in F-theory geometry. The symmetry breaking bundles corresponding to the topology $c_{2}\left(V_{i}\right) \sim \zeta_{i}$ will not be visible in the F-theory geometry, and can only be seen in F-theory when G-flux is correctly incorporated.

In the context of the present investigation, we hope to explore the full moduli/vacuum space of the dual theories including G-flux in future work. For now, however, we simply provide an illustration of where the purely geometric criteria may miss solutions involving exotic G-flux, in an example appearing in section 7.9.

\section{Examples}

We examine some specific examples of F-theory models on bases $\mathcal{B}_{3}$ that are $\mathbb{P}^{1}$ bundles over various bases $B_{2}$ and which illustrate various features discussed in the main part of the paper.

\section{1 $\quad \mathbb{P}^{1}$ bundles over $\mathbb{P}^{2}$}

We begin with the simplest example, taking $\mathcal{B}_{3}$ to be a $\mathbb{P}^{1}$ fibration over $B_{2}=\mathbb{P}^{2}$. The effective divisors on $\mathbb{P}^{2}$ are multiples $n H$ of the hyperplane class $H$ with $H \cdot H=1$. The (anti-)canonical class is $-K_{2}=3 H$. The general constraint on the twist $T=t H$ is that $|t| \leq 18$; this is the analogue of the constraints (4.8)-(4.11) for a curve of self-intersection +1 . This class of F-theory models and their heterotic duals was described in [30, 38].

From the general classification of allowed F-theory models it follows that there is a valid model with $t=18\left(-6 K_{2}-t H=0\right)$, and that there are valid models for $0 \leq t \leq 12$ $\left(-4 K_{2}-t H\right.$ effective). Models with negative $t$ are equivalent under reflection to those with positive $t$. The twists $13 \leq t \leq 17$ correspond to bases $\mathcal{B}_{3}$ in which $g_{5}$ vanishes on some curves on the $\mathfrak{e}_{8}$ locus $\Sigma_{-}$, which must be blown up for a smooth threefold base, analogous to the Hirzebruch surfaces $F_{9,10,11}$ in the $6 \mathrm{D}$ construction. Since the base $B_{2}$ has no curves of self-intersection -2 or below, the base-point free condition is never violated. The resulting $4 \mathrm{D}$ supergravity models have a range of gauge group factors according to the value of $t$. We thus have a total of 14 distinct bases $\mathcal{B}_{3}$ corresponding to different twists in a $\mathbb{P}^{1}$ bundle over $\mathbb{P}^{2}$ giving F-theory models with distinct smooth heterotic duals.

These features can be seen explicitly through a toric construction, where the parts of the fan from $B_{2}$ are

$$
w_{0}=(0,1,0), w_{1}=(1,0,0), w_{2}=(-1,-1, t) .
$$


Here we have used linear transformations to set the component of $T$ to 0 for the first two vectors. The F-theory condition that $f, g$ do not automatically vanish to degrees 4,6 on $\Sigma_{-}$corresponds to the condition that $t \leq 18$ in the toric picture since the plane spanned by $w_{1}, w_{2}, w_{3}$ intersects the third axis at $(0,0, t / 3)$, so $t / 3 \leq 6$. (In general, the condition that $g$ not vanish on a divisor such as $\Sigma_{-}$at degree 6 can be described in any toric case as the condition that the plane spanning the $w_{i}$ 's intersect the $z$ axis at a value less or equal to 6 , as can be verified geometrically. ${ }^{20}$ ) The corresponding condition for $\Sigma_{+}$gives $t \geq-18$.

On the heterotic side, the choice of $t$ corresponds to the bundle decomposition where $\eta_{1,2}=18 \pm t \geq 0$ is the number of instanton factors in each component of the gauge group, which must be nonnegative associated with the condition that $\eta_{i}$ is effective.

Specific examples with generic gauge algebras $\mathfrak{s u}(2)$ and $\mathfrak{s u}(3)$ arise in the cases $t=4,5$. For $t=4, f$ and $g$ vanish to degrees 1,2 on $\Sigma_{-}$, as can be seen from the fact that $f_{0}, g_{0}$, and $g_{1}$ must all vanish as the corresponding divisors $-n K_{2}-(n-k) t H=-4 H,-6 H,-2 H$ are all non-effective. Similarly, for $t=5, f, g$ vanish to degrees $(2,2)$. Both of these cases correspond to situations where the gauge algebra $\mathfrak{s u}(2)$ or $\mathfrak{s u}(3)$ cannot be enhanced to an $\mathfrak{s u}(5)$, though the algebra can be enhanced to the exceptional series $\mathfrak{g}_{2}, \mathfrak{f}_{4}, \mathfrak{e}_{6}, \mathfrak{e}_{7}, \mathfrak{e}_{8}$.

The unique model with an $\mathrm{SO}(32)$ heterotic dual is the model with $t=6\left(t H=-2 K_{2}\right)$, with gauge algebra $\mathfrak{s o}(8)$, parallel to the $6 \mathrm{D}$ model on $\mathbb{F}_{4}$.

\section{2 $\quad \mathbb{P}^{1}$ bundles over $\mathbb{F}_{m}$}

Now consider F-theory models where the base $B_{2}$ is a Hirzebruch surface. Some of these models were also discussed in [30]. The cases $\mathbb{F}_{0}$ and $\mathbb{F}_{1}$ are qualitatively similar to the models described in the previous section, and are always base-point free as there are no -2 curves in the base. The cases $\mathbb{F}_{m}$ with $m>2$ correspond to singular geometries on the heterotic side. We briefly describe the cases $\mathbb{F}_{0}$ and $\mathbb{F}_{1}$ and then focus on the case $\mathbb{F}_{2}$. For any Hirzebruch surface $\mathbb{F}_{m}$, the cone of effective divisors is generated by divisors $S, F$ with $S \cdot S=-m, F \cdot F=0, S \cdot F=1$, and $-K_{2}=2 S+(2+m) F$.

For $\mathbb{F}_{0}$, we have $-K_{2}=2 S+2 F$, and we can parameterize $T=a S+b F$. There are symmetries under $T \rightarrow-T$ and $a \leftrightarrow b$. The general constraints on twists over curves of selfintersection 0 give $-12 \leq a, b \leq 12$. There is a single $\mathfrak{e}_{8}$ model with $a=b=12\left(T=-6 K_{2}\right)$. For all other good bases $-4 K_{2} \pm T$ are effective, so $-8 \leq a, b \leq 8$. Up to symmetries this gives $81+1=82$ distinct twists associated with valid F-theory models. As in the models over $B_{2}=\mathbb{P}^{2}$, there are a variety of gauge groups associated with the different twists.

For $\mathbb{F}_{1}$, we have $-K_{2}=2 S+3 F$, and we can again parameterize $T=a S+b F$. There is a symmetry under $T \rightarrow-T$. The general constraints on twists over curves of self-intersection 0 and -1 give $|a|=T \cdot F \leq 12,|b-a|=T \cdot S \leq 6$. There is a single $\mathfrak{e}_{8}$ model with $a=12, b=18\left(T=-6 K_{2}\right)$. For all other good bases $-4 K_{2} \pm T$ are effective, so $|a| \leq 8,|b| \leq 12$. Up to the sign symmetry of $T$ this gives $108+1=109$ distinct twists associated with valid F-theory models. Again, there are a variety of gauge groups associated with the different twists, and all models have $\eta_{ \pm}=-6 K_{2} \pm T$ base-point free.

\footnotetext{
${ }^{20}$ Thanks to L. Swanson for discussions on this point.
} 
A situation mentioned in section 4.1 .2 occurs for several $\mathbb{P}^{1}$ bundles $\mathcal{B}_{3}$ over $B_{2}=\mathbb{F}_{1}$, where a codimension two singularity arises on a curve despite the absence of gauge groups from codimension one singularities. A sample example of this occurs for the twist $T=2 F$. In this case, $-n K-(n-k) T=2 n S+(n+2 k) F$ has a negative intersection with $S$ when $n>2 k$, so $f_{0}, f_{1}, g_{0}, g_{1}, g_{2}$ all vanish on $S$, giving a $(2,3)$ codimension two singularity type over the curve $S \cap \Sigma_{-}$although there is no gauge group on $\Sigma_{-}$as $-n K-(n-k) T$ are all effective for $n=4,6,0 \leq k<n$.

Now we consider models with $B_{2}=\mathbb{F}_{2}$. We have $-K_{2}=2 S+4 F$, and we parameterize $T=a S+b F$, with a symmetry under $T \rightarrow-T$ exchanging $\Sigma_{ \pm}$. The general constraints on twists over curves of self-intersection 0 and -2 constrain $|a|=T \cdot F \leq 12,|b-2 a|=T \cdot S \leq 1$. There is a single $\mathfrak{e}_{8}$ model with $a=12, b=24\left(T=-6 K_{2}\right)$. The models with $b=2 a$ are base-point free; from the constraint that $-4 K_{2}-T$ be effective the base-point free models have $0 \leq a \leq 8$ up to symmetry, so along with the $\mathfrak{e}_{8}$ model there are 10 base-point free configurations. For the non-base-point free configurations, up to the sign symmetry on $T$ we can choose $b=2 a+1$, from which

$$
-3 K_{2}-T=(6-a) S+(12-2 a-1) F
$$

must be effective, and similar for $-3 K_{2}+T$. This constrains

$$
-8 \leq a \leq 5
$$

so there are 14 non-base-point free configurations, with gauge groups up to $\mathfrak{f}_{4}$ on $\Sigma_{-}$.

We can describe these cases explicitly in toric language. The toric fan for $\mathcal{B}_{3}$ contains the rays

$$
\begin{aligned}
& s_{ \pm}=(0,0, \pm 1) \\
& w_{0}=(0,1,0) \\
& w_{1}=(1,0,0) \\
& w_{2}=(0,-1, a) \\
& w_{3}=(0,-1, b) .
\end{aligned}
$$

The twist $T$ is parameterized by the integers $a, b$. An explicit computation of the monomials in the dual lattice that satisfy $\left\langle m, w_{\alpha}\right\rangle \geq-4,-6$ confirms that the cases described above are the only ones for which the F-theory model is acceptable and that in all these cases $f, g$ have acceptable degrees of vanishing on all divisors and curves.

We consider explicitly the cases where $\eta_{-}=-6 K_{2}-T$ fails the base-point free condition. When $a<2, b=2 a+1$, there is no vanishing of $f, g$ on $\Sigma_{-}$. For the twist combination $(a, b)=(2,5)$, the vanishing degrees are 1,2 , so the gauge algebra contribution from $\Sigma_{-}$ is $\mathfrak{s u}_{2}$. This should correspond on the heterotic side to an $E_{7}$ structure bundle on the Calabi-Yau described by the generic elliptic fibration over $\mathbb{F}_{2}$ that violates the base-point free condition, with $\eta=6 c_{1}-T=10 S+19 F$, which is not base-point free since $\eta \cdot S=-1$.

A similar analysis for the twist combination $(a, b)=(3,7)$ gives vanishing degrees of $f, g$ on $\Sigma_{-} 2,3$, for a gauge algebra factor of $\mathfrak{g}_{2}$ and a dual heterotic bundle structure 
group of $\mathfrak{f}_{4}$. In this case the bundle has $\eta=9 S+17 F$. For the twists $(a, b)=(4,9)$ and $(5,11)$ the vanishing degrees are 3,4 , so the gauge algebra contribution on $\Sigma_{-}$is $\mathfrak{f}_{4}$, and the heterotic structure group is $\mathfrak{g}_{2}$.

This gives a number of explicit examples of F-theory constructions that violate the base-point free condition, where the dual heterotic model should nonetheless exist with a bundle having an exceptional structure group.

\subsection{An F-theory model over $B_{2}=\mathbb{F}_{3}$ with a codimension two singularity but no gauge group}

As discussed in section 3.2, all elliptically fibered Calabi-Yau geometries over the base $B_{2}=\mathbb{F}_{3}$ are singular due to the Kodaira singularity over the -3 curve $D$ in the base with vanishing of $f, g$ to degrees $(2,2)$ or greater. This means that no F-theory models on any base $\mathcal{B}_{3}$ over $B_{2}=\mathbb{F}_{3}$ can have a smooth heterotic dual. In most cases, the absence of the heterotic dual is made particularly clear by the appearance of an additional gauge group factor in the $4 \mathrm{D}$ F-theory model over the divisor in $\mathcal{B}_{3}$ associated with $D$. In the singular heterotic dual theory this would correspond to an additional gauge factor arising at the singularity in the Calabi-Yau geometry. It is interesting to note, however, that for certain values of the twist $T$, a $\mathbb{P}^{1}$ bundle $\mathcal{B}_{3}$ over $B_{2}=\mathbb{F}_{3}$ can be constructed so that there is no extra nonabelian gauge group factor. For example, with the twist $T=F$, there is no divisor that must carry a gauge group factor, though there is a codimension two singularity where $(f, g)$ vanish to degrees $(3,4)$ on the curve $S \cap \Sigma_{-}$. These assertions can easily be checked explicitly using the monomials computed in the toric description.

\section{$7.4 \mathrm{dP}_{2}$}

The second del Pezzo surface, $\mathrm{dP}_{2}$, is constructed by blowing up $\mathbb{P}^{2}$ at two points, giving a pair of exceptional divisors $E_{1}, E_{2}$ with $E_{1} \cdot E_{1}=E_{2} \cdot E_{2}=-1, E_{1} \cdot E_{2}=0$. The proper transform of the line passing through the two points is a third -1 curve $F=H-E_{1}-E_{2}$, with $F \cdot E_{1}=F \cdot E_{2}=1$. The cone of effective divisors is spanned by $F, E_{1}, E_{2}$, which we can write as $(1,-1,-1),(0,1,0),(0,0,1)$ in a basis where the intersection product is $\operatorname{diag}(1,-1,-1) \cdot \mathrm{dP}_{2}$ also has a simple toric presentation, but we use this more abstract formulation for the del Pezzo examples to illustrate how the methods of this paper can be implemented outside the toric context. The (anti-)canonical class of $\mathrm{dP}_{2}$ is the proper transform of $-K=3 H$ on $\mathbb{P}^{2}$,

$$
-K=3 F+2 E_{1}+2 E_{2}
$$

If we parameterize the twist as

$$
T=a F+b E_{1}+c E_{2},
$$

there are symmetries under $b \leftrightarrow c$ and $(a, b, c) \leftrightarrow(-a,-b,-c)$.

We can now count the set of allowed twists $T$ using the conditions described in section 4.1.3 and section 4.1.5. From the analysis in section 4.1.6, we know this gives a necessary and sufficient set of conditions for the set of allowed $\mathcal{B}_{3}$ 's. There is a single twist 
$T=-6 K$ of class (A). There are no -2 curves in the base, so all other $\mathcal{B}_{3}$ 's are of type (B). The constraints that

$$
-4 K \pm T=(12 \pm a) F+(8 \pm b) E_{1}+(8 \pm c) E_{2}
$$

are effective constrain $|a| \leq 12,|b| \leq 8,|c| \leq 8$. The constraints that $\left|T \cdot E_{1}\right|,\left|T \cdot E_{2}\right|,|T \cdot F| \leq$ 6 imply $|a-b| \leq 6,|a-c| \leq 6,|a-b-c| \leq 6$. Up to the symmetries listed above, there are 471 distinct $T$ 's that satisfy these conditions, so the number of distinct $\mathbb{P}^{1}$ bundles $\mathcal{B}_{3}$ over $B_{2}=\mathrm{dP}_{2}$ that give good $\mathrm{F}$-theory models is 472 . This agrees with a direct analysis using toric methods, as described in section 9 .

\section{$7.5 \mathrm{dP}_{3}$}

The story for $\mathrm{dP}_{3}$ is similar to that for $\mathrm{dP}_{2}$. Blowing up $\mathbb{P}^{2}$ at 3 generic points gives 3 exceptional divisors $E_{1}, E_{2}, E_{3}$, and three -1 curves $X_{1}=H-E_{2}-E_{3}, X_{2}=H-E_{1}-$ $E_{3}, X_{3}=H-E_{1}-E_{2}$ from the proper transforms of the lines connecting each pair of points. In a basis with intersection form $\operatorname{diag}(1,-1,-1,-1)$ we have

$$
\begin{array}{ll}
X_{1}=(1,0,-1,-1) & E_{1}=(0,1,0,0) \\
X_{2}=(1,-1,0,-1) & E_{2}=(0,0,1,0) \\
X_{3}=(1,-1,-1,0) & E_{3}=(0,0,0,1)
\end{array}
$$

There are symmetries under the 6 permutations of the indices $i=1,2,3$, and under $E_{i} \leftrightarrow$ $X_{i}$, which maps

$$
\left(a,-b_{1},-b_{2},-b_{3}\right) \leftrightarrow\left(2 a-b_{1}-b_{2}-b_{3}, a-b_{2}-b_{3}, a-b_{1}-b_{3}, a-b_{1}-b_{2}\right)
$$

In the toric picture this can be seen as the 12-fold dihedral symmetry group $D_{6}$ of the regular hexagon. A divisor $D=\left(a,-b_{1},-b_{2},-b_{3}\right)$ is effective if $a \geq 0, b_{1}+b_{2}+b_{3} \leq 2 a$. The (anti-) canonical class of $B_{2}=\mathrm{dP}_{3}$ is again the proper transform of $H$

$$
-K=(3,-1,-1,-1)=\sum_{i} X_{i}+\sum_{i} E_{i} .
$$

There is one base $\mathcal{B}_{3}$ of type (A), with $T=-6 K$. To enumerate bases $\mathcal{B}_{3}$ of type (B), following the analysis of section 4.1.6, it is sufficient to identify all twists $T=\left(a,-b_{1},-b_{2},-b_{3}\right)$ so that the local twist conditions

$$
\begin{aligned}
& \left|T \cdot E_{i}\right|=\left|b_{i}\right| \leq 6 \\
& \left|T \cdot X_{i}\right|=\left|a-b_{j}-b_{k}\right| \leq 6, \quad i, j, k \text { distinct } \in\{1,2,3\}
\end{aligned}
$$

are satisfied and $-4 K \pm T=\left(12 \pm a,-4 \mp b_{1},-4 \mp b_{2},-4 \mp b_{3}\right)$ is effective, which implies

$$
|a| \leq 12, \quad\left|2 a-b_{1}-b_{2}-b_{3}\right| \leq 12 .
$$

A simple enumeration shows that up to the $D_{6}$ symmetry group there are 775 solutions of all these conditions, so a total of 776 distinct possible bases $\mathcal{B}_{3}$ that are $\mathbb{P}^{1}$ bundles over $\mathrm{dP}_{3}$. As for $\mathrm{dP}_{2}$, this result agrees with the explicit enumeration done using toric methods described in section 9 . 


\section{$7.6 \mathrm{dP}_{4}$}

For $\mathrm{dP}_{n}$ the analysis is again similar to $\mathrm{dP}_{3}$ and $\mathrm{dP}_{2}$, though there is no toric construction, there are more symmetries, and the effectiveness condition is increasingly complicated as the number of -1 curves on the wall of the cone of effective divisors increases.

For $\mathrm{dP}_{4}$ there are 4 exceptional divisors $E_{i}, E_{1}=(0,1,0,0,0), \ldots E_{4}=(0,0,0,0,1)$, and 6 proper transforms of lines $X_{i j}, X_{12}=(1,-1,-1,0,0), \ldots$. The intersection form is $\operatorname{diag}(1,-1,-1,-1,-1)$, with (anti-) canonical class $-K=(3,-1,-1,-1,-1)$. There are symmetries under arbitrary permutations of the $i$ 's, as well as additional symmetries of the form

$$
E_{1} \leftrightarrow X_{23}, \quad E_{2} \leftrightarrow X_{13}, \quad E_{3} \leftrightarrow X_{12}, \quad X_{i 4}, E_{4} \text { fixed }
$$

The full symmetry group is of order $5 !=120$, and can be seen most clearly by redefining $\tilde{X}_{0 i}=E_{i}, \tilde{X}_{i j}=\frac{1}{2}\left|\epsilon_{i j k l}\right| X_{k l}$, for which the nonzero intersection products are $\tilde{X}_{\mu \nu} \cdot \tilde{X}_{\mu \nu}=-1, \tilde{X}_{\mu \nu} \cdot \tilde{X}_{\lambda \sigma}=1$ when $\mu, \nu, \lambda, \sigma \in\{0,1,2,3,4\}$ are distinct; in terms of the $\tilde{X}$ 's, the symmetry group is simply the set of permutations on all 5 possible index values.

In $\mathrm{dP}_{4}$, a divisor $D=\left(a,-b_{1},-b_{2},-b_{3},-b_{4}\right)$ is effective iff $b_{i} \leq a \forall i, \sum_{i} b_{i} \leq 2 a$, which can be seen from the conditions that $D$ must be formed from a positive integral linear combination of $X_{i j}$ 's and $E_{i}$ 's. The twist $T=\left(s, t_{1}, t_{2}, t_{3}, t_{4}\right)$ must satisfy the conditions

$$
\left|T \cdot E_{i}\right|=\left|t_{i}\right| \leq 6, \quad\left|T \cdot X_{i j}\right|=\left|s-t_{i}-t_{j}\right| \leq 6 .
$$

As in the previous del Pezzo examples, there is one solution with $T=-6 K$, and we can enumerate all solutions with $-4 K \pm T$ effective, which along with the twist conditions (4.8)(4.11) give necessary and sufficient conditions for a good base $\mathcal{B}_{3}$. An explicit enumeration shows that after taking account of symmetry there are 6976 distinct bases $\mathcal{B}_{3}$ of type (B), for a total of $6977 \mathcal{B}_{3}$ that are $\mathbb{P}^{1}$ fibrations over $\mathrm{dP}_{4}$.

For higher $\mathrm{dP}_{n}$, and for generalized del Pezzo surfaces, the analysis can be carried out in a similar fashion. As $n$ increases, however, the details of the calculation become more complicated. For $\mathrm{dP}_{5}$, for example, there is an additional -1 curve from a conic passing through all 5 blown up points, $C=2 H-E_{1}-E_{2}-E_{3}-E_{4}-E_{5}$, which complicates the effectiveness condition on divisors. In principle, however, for any base $B_{2}$, the number of twists satisfying the local twist conditions is finite, and the determination of the full set of $\mathcal{B}_{3}$ 's over $B_{2}$ can be done efficiently and explicitly.

\subsection{An example of an upper and lower bound on $\eta$}

One of the more novel observations of this study is the fact that for certain fourfold geometries there exist generic symmetries that can be neither broken (Higgsed) or enhanced at any points in the complex structure moduli space of $Y_{4}$. These restrictions arise because of a variety of features, however all the failures of "enhancement" occur because of too-high a degree of vanishing of $(f, g)$ on divisors and curves as described in section 6 .

The consequences of having a twist $T$ of the $\mathbb{P}^{1}$-fibered base $\mathcal{B}_{3}$ on the F-theory side that gives rise to such a restrictive condition on the gauge group corresponds in the heterotic geometry to a choice of partial bundle topology $\eta$ for which only one structure group $H$ is possible (subject once again to the caveats arising from ignoring G-flux, see section 6.5) 
Let us consider here an example of this type for which only one symmetry is possible and all Higgsing/enhancing is forbidden. This is the case for the base $\mathcal{B}_{3}$ defined by the $\mathbb{P}^{1}$ bundle with twist $T=5 S+11 F$ on $\mathbb{F}_{2}$. Constructing the generic Weierstrass model over this base $\mathcal{B}_{3}$, it is straightforward to verify that this $Y_{4}$ manifests a generic $F_{4}$ symmetry.

In the dual heterotic theory, this corresponds to a $G_{2}$ bundle over the threefold $X_{3}$ with $\eta=7 S+13 F$. More precisely, the heterotic bundle $V_{1}$ has $\eta_{-}=7 S+13 F$ and is a $G_{2}$ bundle. The second bundle, associated to $\eta_{+}$, has generic $E_{8}$ structure group and hence one $E_{8}$ factor is generically completely broken and will not concern us further. More explicitly, we have the following Weierstrass equation for $Y_{4}$ :

$$
Y^{2}=X^{3}+\left(f_{3} z^{3}+f_{4} z^{4}+\ldots\right) X+\left(g_{4} z^{4}+g_{5} z^{5}+g_{6} z^{6}+\ldots\right)
$$

(Here $(X, Y, Z=1)$ are the coordinates on the elliptic fiber of the $C Y_{4}$, while $z=0$ defines the 7 -brane locus (i.e., the section $\Sigma_{-}$) inside of $\mathcal{B}_{3}$ and is of $E_{6} / F_{4}$ type according to Kodaira-Tate.) As mentioned in previous sections, if $g_{4}$ is a perfect square then the symmetry is $E_{6}$, and for more general polynomials it is $F_{4}$.

For the twist $T=5 S+11 F,(7.22)$ is the generic form of the Weierstrass model for arbitrary complex structure. The fact that the $F_{4}$ symmetry is generic (i.e., cannot be Higgsed) from the point of view of the F-theory Weierstrass model has the same natural low-energy $4 \mathrm{D}$ interpretation on both sides of the duality — there is simply no charged matter available to get a vev in vacuum.

For the given twist, all additional tunings of the complex structure that might increase the gauge group on $\Sigma_{-}$induce non-CY singularities. As an example, consider the specialization of $g_{4}=\alpha^{2}$ for some polynomial $\alpha$ of the appropriate degree. Here the vanishing degree of $(f, g, \Delta)$ increases from the generic values of $(4,5,10)$ on the curve $\Sigma_{-} \cap S$ to $(4,6,12)$ on the same curve, and hence the singularity cannot be resolved without going to a different $\mathrm{F}$-theory base $\mathcal{B}_{3}$ by blowing up the curve.

In this case, the restriction on enhancement has a clear interpretation in terms of the heterotic bundle geometry. An enhancement of the symmetry of $F_{4} \rightarrow E_{6}$ for example, corresponds in the heterotic theory to a reduction in rank of the associated bundle from $G_{2} \rightarrow \mathrm{SU}(3)$. For the case at hand this would indicate that by tuning the complex structure of $Y_{4}$ we were inducing a "splitting" of the vector bundle. In terms of the associated vector bundles the following reduction of representations in $G_{2} \rightarrow \mathrm{SU}(3)$,

$$
\mathbf{7} \rightarrow \mathbf{3}+\overline{\mathbf{3}}+\mathbf{1}
$$

would lead to

$$
V_{7}=V_{3} \oplus V_{3}^{\vee} \oplus \mathcal{O}_{X_{3}}
$$

the fact, however, that this tuning leads to a badly singular $Y_{4}$ indicates that a generic $G_{2}$ bundle with $\eta=7 S+13 F$ cannot be decomposed as in (7.24) for smooth $\mathrm{SU}(3)$ bundles $V_{3}$. Instead, any such decomposition must lead to non-locally free sheaves (i.e., heterotic "small instantons" [117-119]) and a degenerate limit of the theory.

For this choice of twist, we have an additional confirmation of this heterotic result in the fact that $\eta$ is not base-point-free. Thus by the arguments of section 6 , we cannot define 
any smooth $\mathrm{SU}(3)$ spectral cover bundle to play the role of $V_{3}$ in (7.24). Although the spectral cover construction is not guaranteed to be representative for the bundle moduli space in general, the consistency conditions on $Y_{4}$ applied to these "non-enhanceable" geometries indicate that if a generic symmetry $F_{4}$ is not base-point free, it will be impossible to enhance the symmetry for special values of the complex structure (compatible with a CY resolution). This provides an interesting window into the moduli space of all such $G_{2}$-bundles by providing general restrictions on possible decompositions like the one given above.

\subsection{Examples with non-trivial chiral matter}

In the previous examples we have seen that the F-theory fourfold geometry frequently encodes otherwise hard to obtain information about the moduli space of vector bundles on heterotic CY threefolds. In this section, we use the heterotic theory to obtain new information about the matter spectrum of a $4 D$ effective F-theory. To accomplish this, we return to the formulas for chiral matter given in section 5.2.

As an example, let us consider $E_{6}$ theories in the dual heterotic/F-theory geometry. As discussed in section 5.3.2, for an $\mathrm{SU}(N)$ bundle described via a spectral cover the chiral index is $[45,103]$

$$
\operatorname{Ind}(V)=-h^{1}(X, V)+h^{1}\left(X, V^{\vee}\right)=\lambda[\eta] \cdot\left[\eta+N K_{2}\right]
$$

where $\lambda$ is defined by (5.27) and (5.28). Thus, the chiral index is proportional to a simple geometric intersection of the curve $[\eta]$ in the base with the matter curve $\left[\eta+N K_{2}\right]$.

To understand the significance of this geometry, the case of $E_{6}$ theories is particularly interesting because for $\mathrm{SU}(3)$ bundles described as spectral covers we can guarantee that the constant $\lambda$ is non-vanishing. Recall from (5.28) that for some integer $m$

$$
\lambda= \begin{cases}m+\frac{1}{2}, & \text { if } N \text { is odd } \\ m, & \text { if } N \text { is even }\end{cases}
$$

Thus, for $\mathrm{SU}(3)$ bundles $/ E_{6}$ theories it is required that $\lambda \neq 0$ and the question of whether or not the theory has chiral matter can be reduced to a of question of intersection theory for the matter curve $\left[\eta+3 K_{2}\right]$ in the 2 -fold base. We will be interested in whether or not this curve is reducible and whether or not it has non-trivial intersection with $[\eta]$.

To illustrate the possibilities, we can consider the four generic $E_{6}$ theories over the base $B_{2}=\mathbb{F}_{1}$. There the twists

$$
T=n S+9 F \quad, \quad 3 \leq n \leq 6
$$

all give rise to $E_{6}$ symmetries on $\Sigma_{-}$. This is easy to check since the coefficient $g_{4}$ in the Weierstrass equation

$$
Y^{2}=X^{3}+\left(f_{3} z^{3}+f_{4} z^{4}+\ldots\right) X+\left(g_{4} z^{4}+g_{5} z^{5}+g_{6} z^{6}+\ldots\right)
$$

satisfies $g_{4} \in H^{0}\left(B_{2}, \mathcal{O}(\eta)^{\otimes 2} \otimes K_{2}^{\otimes 6}\right)=H^{0}\left(B_{2}, \mathcal{O}\left(-6 K_{2}-2 T\right)\right)=H^{0}\left(\mathbb{F}_{1}, 2(6-n) S\right)$, which indicates that $g_{4}$ is a perfect square in these $\operatorname{cases}^{21}$ and hence (as described in the previous

\footnotetext{
${ }^{21}$ Note that the line bundle cohomology over $\mathbb{F}_{n}$ can be shown to satisfy: $h^{0}\left(\mathbb{F}_{n}, \mathcal{O}(S)\right)=1$ for the divisor $S^{2}=-n$. Hence if $s$ is the toric coordinate associated to the divisor $S$, the generic (only) element of $H^{0}\left(\mathbb{F}_{n}, n S\right)$ is $s^{n}$.
} 
section) the fiber type is split to $E_{6}$ (rather than the generic, non-split $F_{4}$ ). Moreover, for $3 \leq n \leq 6$ we have the heterotic topology:

$$
\eta=(12-n) S+9 F \quad \text { and } \quad \eta+3 K_{2}=(6-n) S
$$

For these $\mathrm{SU}(3)$ bundles, $\eta$ is effective and base-point-free, $\eta-3 c_{1}$ is effective, and it is straightforward to verify that simple line bundles $L_{\mathcal{S}}$ of the form (5.27) can be found with $\lambda \neq 0$. Thus, we are guaranteed that smooth spectral cover bundles exist (stable in the appropriate adiabatic region in Kähler moduli space).

In the case that $g_{4}=\alpha^{2}$ the polynomials in (7.28) appear as a $\mathrm{SU}(3)$ spectral cover inside the heterotic $C Y_{3}$ of the form

$$
g_{5} \hat{Z}^{3}+f_{3} \hat{X} \hat{Z}+\alpha \hat{Y}=0
$$

where $(\hat{X}, \hat{Y}, \hat{Z})$ are coordinates on the $C Y_{3}$ elliptic fiber (the above equation gives three points on the elliptic fiber for each point on the $\mathbb{F}_{2}$ base, as expected for an $\mathrm{SU}(3)$ spectral cover). As usual, $f_{4}, g_{6}$ in the fourfold Weierstrass equation above appear as the coefficients in the $C Y_{3}$ Weierstrass

$$
\hat{Y}^{2}=\hat{X}^{3}+f_{4} \hat{X}+g_{6}
$$

Recall from the arguments of section 5.3.2 that the coefficient $\alpha \in H^{0}\left(\mathcal{O}(\eta) \otimes K_{2}^{\otimes 3}\right)=$ $H^{0}\left(\mathbb{F}_{2}, \mathcal{O}((6-n) S)\right.$ in $(7.30)$ defines the "matter curve", $\alpha=0$. This is where the 27 -type matter is localized in both the heterotic/F-theory geometries.

Thus, for this class of bundles (5.28) and (5.32) can be used to straightforwardly compute

$$
\operatorname{Ind}(V)=\text { no. of } \overline{\mathbf{2 7}}^{\prime} s-\text { no. of } \mathbf{2 7}^{\prime} s=(6-n)(n-3)
$$

From this we see that the cases $n=3$ and $n=6$ have chiral index zero, but for $n=4,5$ the theory must have chiral matter. Given the full defining data of the bundle, the exact multiplicity of the 27's and $\overline{\mathbf{2 7}}$ s could be computed using Leray spectral sequences [98], but even at this preliminary level, the results of the chiral index are intriguing. For these dual geometries, given a value of $\eta$, it can immediately be determined whether or not the theory contains chiral matter.

Of course, we derived the necessity of a non-vanishing chiral index for an $\mathrm{SU}(3)$ bundle described as a spectral cover, but the third Chern class (and hence the Chiral index) is a topological invariant in the bundle moduli space. As a result, so long as a good SU(3) spectral cover bundle exists, we can use it as a probe to extract the structure of the full moduli space of hermitian bundles, all of which must have non-vanishing index! As discussed in section 6.4, all CY fourfold geometries with generic $E_{6}$ symmetry and smooth heterotic duals satisfy the base-point-free condition and can be described by well-behaved spectral cover bundles as in section 5.3.

It would be interesting to investigate the question of chiral matter in this context more directly on the F-theory side in the future, in particular by including G-flux. Some general aspects of how G-flux can be incorporated in 4D F-theory models are described in [120-123]. Progress has been made in understanding how chiral matter in F-theory 
models can be determined in the presence of G-flux based on aspects of the spectral cover construction [20, 124-130] and more directly from the M-theory description [131], but a more complete and directly computable formulation is desirable. An inspection of (7.28) shows that the points determined by the intersection

$$
[\eta] \cdot\left[\eta+N K_{2}\right]
$$

in the F-theory geometry corresponds exactly to the simultaneous vanishing of $g_{4}$ and $g_{5}$ at points in the base - that is, the chiral index in these cases is counted by co-dimension 3 singular loci in the 4-fold geometry. The observation that chiral matter and co-dimension 3 singularities (and associated $G$-flux) could be linked (at least in $K 3$-fibered 4 -folds) has been observed for some time $[124,125]$ and has been used more recently in F-theory model building [20, 132].

It would be interesting to study more generally whether simple correlations such as those between (5.21) and (5.28) and (5.32) exist between $\eta$ and the chiral index, independent of the existence of a heterotic dual. In fact, it is possible that the F-theory 4-fold could explicitly give indications of such correlations through its topology. For example, it is well known that some 4-folds cannot be good F-theory vacua without including non-trivial G-flux. In these cases their second Chern class (or more generally Wu class) is incompatible with trivial G-flux in the presence of quantization conditions. A study of the topology of $Y_{4}$ and its links to intersection structure such as that in (7.25) could yield important information along these lines (for similar investigations see $[133,134]$ which explore Chern and $\mathrm{Wu}$ classes of $Y_{4}$ with simple singularities). We hope to explore this in future work.

\subsection{Generic G-flux that breaks gauge symmetry}

In section 6.5, we discussed how G-flux can break the gauge group associated with a purely geometric construction, through the structure of the second Chern class of the dual heterotic bundle. As an example of this mechanism, consider the case of base $B_{2}=\mathbb{P}^{1} \times \mathbb{P}^{1}$ with twist $T=6 c_{1}\left(B_{2}\right)$; i.e., $\eta_{-}=0$. According to the arguments of section 3 , we would naturally have determined from the F-theory geometry $Y_{4}$ that $\Sigma_{-}$carried an $E_{8}$ symmetry (i.e., the fiber degeneration is type $\mathrm{II}^{*}$ ) and hence that there were no smooth bundles $V_{1}$ on $X_{3}$ with $\eta_{-}=0$ (i.e., that $V_{1}$ is trivial and $V_{2}$ satisfies $\eta_{+}=12 c_{1}\left(B_{2}\right)$ ). However, this is too quick since this argument ignores the fact that $\eta$ alone is not enough to determine even $c_{2}\left(V_{1}\right)$. To see this, consider the following smooth heterotic geometry.

On the base $B_{2}$, consider the poly-stable rank 2 vector bundle defined as a kernel (of the map $m$ ) via the following short exact sequence (i.e., a "monad" bundle [108]):

$$
0 \rightarrow \mathcal{V}_{1} \rightarrow \mathcal{O}(0,1)^{\oplus 2} \oplus \mathcal{O}(1,0)^{\oplus 2} \stackrel{m}{\rightarrow} \mathcal{O}(1,1)^{\oplus 2} \rightarrow 0
$$

(where $\mathcal{O}(a, b)=\mathcal{O}(a S+b F)$ and $S, F$ are the hyperplanes in each $\mathbb{P}^{1}$ factor). For an appropriately block-diagonal choice of the map $m, \mathcal{V}_{1}$ is a simple twist of the poly-stable tangent bundle of $\mathbb{F}_{0}$ with vanishing slope; i.e., $\mathcal{V}_{1}=T \mathbb{P}^{1} \otimes \mathcal{O}(1,1)$. For generic choices of $m$ this bundle is slope-stable for all of the Kähler cone of $\mathbb{P}^{1} \times \mathbb{P}^{1}$. Over the entire elliptically fibered threefold, $\pi: X_{3} \rightarrow B_{2}$, we can likewise define the stable, slope-zero pull-back 
bundle $\pi^{*}\left(\mathcal{V}_{1}\right)=V_{1}$ with $c_{1}\left(V_{1}\right)=0$ and $c_{2}\left(V_{1}\right)$ non-trivial solely from the pull-back of the $(2,2)$-form on the base, $\pi^{*}(\zeta)$

$$
c_{2}\left(V_{1}\right)=\pi^{*}(\zeta)=\pi^{*}\left(4 \omega_{1} \omega_{2}\right) \quad \Rightarrow \eta_{-}=0
$$

where $\omega_{i}$ are the $(1,1)$-forms dual to the divisors $S, F$ in $\mathbb{F}_{0}$.

The pull-back bundle defined by (7.34) is an example of a smooth, everywhere stable $\mathrm{SU}(2)$ bundle which breaks $E_{8} \rightarrow E_{7}$ in the heterotic effective theory. Thus, in contradiction to the conventional indication of the F-theory $E_{8}$-type Weierstrass equation, it is clear that this is an everywhere well-defined $E_{7}$ theory. In the other $E_{8}$ factor a generic bundle with $c_{2}\left(V_{2}\right)=12 c_{1}\left(\mathbb{F}_{0}\right)+88 \pi^{*}\left(\omega_{1} \omega_{2}\right)$ (as required by anomaly-cancellation, see (5.2) and (A.7)) breaks all the symmetry. At first pass it would seem that the bundle in (7.34) cannot be naively be described by a smooth spectral cover and that as a result the Heterotic/F-theory dictionary is unclear. For spectral covers $\eta=0$ indicates that $[\mathcal{S}]=2[\sigma]$ from $(5.24)$ and we would be tempted to conclude here that the spectral cover (5.25) with $\eta=0$ described only the Fourier-Mukai transform of the trivial rank 2 bundle $\mathcal{O}^{\oplus 2}$. However, this is forgetting half of the data of the Fourier-Mukai transform: in particular the rank 1 sheaf $L_{\mathcal{S}}$ over $\mathcal{S}$ (see section 5.3 and (5.27)). Taking into account the possibility of rank 1 sheaves on the non-reduced scheme $\mathcal{S}$ which arise from higher rank sheaves (in this case rank 2) on $\sigma=0[135,136]$ it is clear that more general bundles $V_{1}$ are possible after FM transform. ${ }^{22}$ In the standard heterotic/F-theory dictionary, the data of these rank 1 sheaves (whether ordinary line bundles or higher rank sheaves in the non-reduced or reducible case as above) is mapped into G-flux [20, 42, 80].

As this example illustrates, such possibilities must be taken into account if one hopes to fully determine the properties of heterotic vector bundle moduli space from its F-theory dual. For now, we consider only the data of $Y_{4}$ itself, focusing on purely geometric structure and properties, and leave an investigation of the intriguing possibilities of G-flux for future work.

\section{Consequences for heterotic bundles}

Many of the new results in this paper are conclusions/constraints regarding properties of the moduli space of bundles (more precisely, the moduli space of semi-stable sheaves) arising in heterotic theories and links between bundle topology and structure group. In this section we provide a brief summary of these results.

Unlike in six dimensions, four-dimensional heterotic/F-theory duality provides new and non-trivial insight into the structure of the heterotic moduli space $\mathcal{M}_{\omega}(c(V))$ of semi-stable sheaves with fixed topology on $X_{3}$. At present, very few techniques are known for determining the dimension and structure of $\mathcal{M}_{\omega}$ on Calabi-Yau threefolds and there are many open questions which are of interest to both physics and mathematics. These include applications to string phenomenology (for example the large scale scans for "Standard

\footnotetext{
${ }^{22}$ As mentioned in section section 5.3.3, the holomorphic tangent bundle to an elliptically fibered threefold, $T X_{3}$, is frequently found to have a degenerate spectral cover description of this type (i.e., reducible or nonreduced $\mathcal{S}$ ) $[110,137]$.
} 
Model" bundles undertaken in [15-17]) as well as more mathematical questions such as the possible existence of new mathematical rules for linking topology $(c(V))$ to conditions for vanishing/triviality of $\mathcal{M}_{\omega}(c(V))$ and the computation of higher rank Donaldson-Thomas invariants. For elliptically fibered Calabi-Yau threefolds, heterotic/F-theory duality provides a rich set of new computational tools and we view this work as a preliminary step in using these tools to determine the full structure of $\mathcal{M}_{\omega}$. We briefly summarize here the main new results:

\section{Effectiveness conditions on $\eta$.}

- As discussed in section 5.1 and section 5.3, the parameter $\eta$ determining part of the second Chern class of the heterotic bundles must obey several effectiveness constraints in different contexts. $\eta$ must be effective in the stable degeneration limit, and $\eta-$ $N c_{1}\left(B_{2}\right)$ must be effective for a spectral cover construction of an $\mathrm{SU}(N)$ bundle. We have found that these constraints are more general. For any F-theory construction with a smooth heterotic dual, $\eta$ must be effective, and $\eta-N c_{1}$ must be effective for $N=2,3$ for gauge groups $E_{7}, E_{6}$ (or smaller) corresponding to heterotic theories with structure bundles $\mathrm{SU}(2), \mathrm{SU}(3)$, independent of the stable degeneration limit or method of bundle construction.

\section{Base-point-freeness and bundles with exceptional structure group.}

- Previous work aimed at describing vector bundles over Calabi-Yau threefolds in the context of heterotic/F-theory duality, such as [19], has focused on bundles with $\mathrm{SU}(N)$ and $\operatorname{Sp}(N)$ structure groups, constructed using spectral covers in the stable degeneration limit. Here we have considered consistency conditions on topology in a construction-independent way and demonstrated that the base-point-freeness condition on $\eta$ is necessary for $\mathrm{SU}(N)$ and $\mathrm{Sp}(N)$ structure groups, independent of the the method of bundle construction.

Moreover, our study has shown that these constraints on the topology of the vector bundle do not seem to be universal. We have considered a broader class of heterotic/F-theory dual models and identified a large range of models in which the base-point free condition on the components $\eta$ of the second Chern class need not be satisfied in the dual F-theory model. In all these models the structure group on the heterotic side is an exceptional group or $\mathrm{SO}(8)$. Thus, F-theory allows us to identify the conditions on the second Chern class that are necessary, and apparently sufficient, for vector bundles with exceptional and $\mathrm{SO}(8)$ structure group to be constructed over a broad class of Calabi-Yau threefolds. These results could be mathematically useful in explicitly constructing or characterizing such bundles. In particular, it would be intriguing to utilize these conditions in formulating topological consistency conditions for bundles constructed through the cameral cover construction (which are at present not as explicitly described as those for the spectral cover construction). 
- One interesting feature of the heterotic models with F-theory duals where the base-point free condition is violated is that they all involve elliptic fibrations over generalized del Pezzo surfaces that contain curves of self-intersection -2 . These surfaces are limits of usual del Pezzo surfaces where the points where $\mathbb{P}^{2}$ is blown up are brought together in specific ways. In principle, the Calabi-Yau threefolds formed over generalized del Pezzo surfaces should simply be special limits in the moduli space of the generic elliptically fibered Calabi-Yau threefold over the corresponding del Pezzo. This limit can be controlled precisely and may provide an avenue for the explicit construction of the vector bundles with exceptional structure groups that arise in these cases. More precisely, in these limits as the complex structure of $B_{2}$ is tuned to produce the generalized del Pezzo surfaces, the Mori cone of effective divisors in $B_{2}$ jumps discontinuously (though $h^{1,1}\left(X_{3}\right)$ remains unchanged), and the Kähler cone of the Calabi-Yau threefold decreases correspondingly. This change in the Kähler cone impacts the properties of the moduli space of stable bundles that can arise, and also seems to restrict the existing bundles to have exceptional structure groups in many cases. ${ }^{23}$

\section{$\mathrm{SO}(32)$ heterotic/F-theory duality and the connectivity of string moduli space.}

- By using topological terms in the $4 \mathrm{D}$ effective supergravity action to characterize heterotic F-theory duality [9], we can identify topologically which F-theory models are dual to heterotic models for $\mathrm{SO}(32)$ as well as $E_{8} \times E_{8}$ models without requiring a stable degeneration limit. We have explicitly identified those F-theory models that are dual to $\mathrm{SO}(32)$ heterotic string theory over a general smooth elliptically fibered Calabi-Yau threefold base, and shown that in all such cases the generic model has a gauge group of $\mathrm{SO}(8)$, which cannot be broken further by Higgsing.

- On the F-theory side, all the models we have considered are connected in a smooth geometric moduli space. Over each base $\mathcal{B}_{3}$ there is a moduli space of Weierstrass models that provides a nonperturbative completion of the perturbative heterotic moduli space of bundles (sheaves) over the dual heterotic elliptically fibered Calabi-Yau threefold. Furthermore, the distinct bases are connected by tensionless string transitions that correspond to small instanton transitions on the heterotic side. For those F-theory bases with $\mathrm{SO}(32)$ heterotic duals, there are also $E_{8} \times E_{8}$ duals; Ftheory/heterotic duality may illuminate the connection between these two distinct heterotic perturbative limits and the resulting relationship on Calabi-Yau threefolds between the moduli spaces of $\mathrm{SO}(8)$ structure group bundles and other bundle structures associated with the $E_{8} \times E_{8}$ theory. This extends to four dimensions results on the geometry of $E_{8} \times E_{8} / \mathrm{SO}(32)$ dual heterotic pairs that were previously understood in higher-dimensional contexts [21, 22, 56, 143-146].

\footnotetext{
${ }^{23}$ See [103, 138-142] for similar "Noether-Lefschetz" type-problems and "jumping" in complex structure/bundle moduli space.
} 


\section{Upper bounds on $H$.}

- As first explored in $[37,38]$, the presence of generic, non-Higgsable symmetries for singular $Y_{4}$ geometries indicates that for a given $\eta$ there is an upper bound on the size of the structure group $H$ for any bundle in the moduli space. As explained in section 6 , if a generic symmetry $G$ cannot be Higgsed in the 4-dimensional effective theory, this implies that for the given topology $(\eta)$ there exist no bundles with structure group larger than $H$, the commutant of $G$ in $E_{8}$. Phrased differently, in order to define a bundle with structure group $H$ over the elliptically fibered CY threefold, there is a minimum "size" for $\eta$. These conditions (given in the absence of $G$-flux) are listed in table 3.

- We further observe that these rules are at present only a first step in determining $\mathcal{M}_{\omega}(c(V))$ and its constraints. As described in section 6.5, for some choices of bundle/4-fold topology it may be that generic $G$-flux breaks the apparent symmetry $G$ indicated by the Weierstrass equation of $Y_{4}$. In these cases, the bundle structure group may be bigger than indicated by the bounds on $\eta$ in table 3 . When this occurs, it must involve non-trivial values for $\zeta$ in $(3.2)$; this indicates a new layer of structure linking not only $\eta$ with $H$, but also with $\left(\zeta, c_{3}(V)\right)$ - the two integer values specifying the remaining bundle topology not studied in this work. Such a correlation would involve a finer level of structure linking $H$ and $c(V)$ than has been so far explored in the literature. We hope to explore these issues in further work.

Lower bounds on structure group, $\boldsymbol{H}$. In addition to the non-Higgsable symmetries described above and their heterotic consequences, in the F-theory geometry we have seen many examples of $Y_{4}$ with a generic symmetry $G$ which cannot be consistently enhanced (see section 6). In the dual heterotic geometry, these geometric observations provide constraints on when a given vector bundle can be consistently decomposed into a reducible sum:

$$
V \rightarrow \mathcal{V}_{1} \oplus \mathcal{V}_{2} \oplus \mathcal{O} \oplus \ldots
$$

with a smaller (reduced) structure group.

- The results of section 6.4 indicate that the base-point-free condition described in section 5.3 and section 6.4 cannot be consistently violated in the case of $H=\mathrm{SU}(N)$ (i.e., for a hermitian bundle, regardless of the method of construction). As a result, for any technology applied to a bundle associated with a consistent non-base-point free choice of $\eta$, it is clear that there is no way to reduce the structure group to $\mathrm{SU}(N)$. We find that all the non-base-point free examples of $\eta$ correspond to structure groups $H=\mathrm{SO}(8), G_{2}, F_{4}, E_{6}, E_{7}$ or $E_{8}$. In these cases, the bundle structure group can never be consistently decomposed $H \rightarrow H_{1} \times H_{2} \ldots$ with $H_{i} \subset H$ hermitian; in these cases the gauge group $G$ thus cannot be enhanced to $E_{6}$ or $E_{7}$.

- In a similar spirit, for many examples (see section 4.2.1) there are generic $\mathrm{SU}(3)$ or $\mathrm{SU}(2)$ symmetries on $Y_{4}$, with $(f, g)$ vanishing to degree $(2,2)$ or $(1,2)$ and no possible enhancements to higher $\mathrm{SU}(N)$ gauge symmetries are possible (this would 
require $(f, g)$ non-vanishing and $\Delta$ vanishing to degree $N)$. As in the case of the non-base-point free examples above, this constrains the ways in which bundles with exceptional structure groups can be decomposed into hermitian factors.

- We find examples for which there appear to be both upper and lower bounds on $H$; in these cases for the given value of $\eta$ the moduli space of semi-stable sheaves can contain bundles with exactly one allowed structure group only. See for example section 7.7, where an example of a topology is given for which $F_{4}$ is the only consistent structure group.

- The lower bounds on $H$ constrain the structure of possible sub-sheaves $\mathcal{F} \subset V$, and determine a bound below which $V$ cannot decompose as $V=\mathcal{F} \oplus V / \mathcal{F}$, etc. The determination of such substructure has important consequences for the Harder-Narasimhan filtration of $V$, and the group quotient structure of $\mathcal{M}$ itself [72].

- Finally, these "lower" bounds on $H$ may be strengthened by the presence of $G$-flux. While $G$-flux cannot enhance the gauge group in a way that violates these lower bounds on $H$, it could act to reduce the generic gauge symmetry arising purely from geometry, which could lead to even stronger lower bounds on $H$ in some cases.

\section{Enumeration of heterotic/F-theory dual pairs with toric bases $\boldsymbol{B}_{2}$}

We have systematically analyzed all toric F-theory bases $\mathcal{B}_{3}$ (constructed as $\mathbb{P}^{1}$ bundles) that have smooth heterotic duals on Calabi-Yau threefolds that are elliptically fibered with section. The toric bases $B_{2}$ that support such models are the generalized del Pezzo (gdP) surfaces, a subset of 16 of the complete set of 61,539 toric surfaces enumerated in [60] that can act as bases for elliptically fibered Calabi-Yau manifolds with section. Over the 16 toric gdP bases we find 4962 distinct $\mathcal{B}_{3}$ 's that have smooth heterotic duals. Each choice of $\mathcal{B}_{3}$ corresponds to a specific Calabi-Yau threefold $X_{3}$ giving the generic elliptic fibration over $B_{2}$, with a particular choice in the part of the bundle topology characterized by $\eta_{i}$ in (3.2). The analysis was performed by considering all possible twists $T$ compatible with the bounds (4.8)-(4.10), and explicitly analyzing the monomial and singularity structure of the resulting Weierstrass model in the toric description. For each model that does not have a $(4,6)$ singularity on a divisor or curve, we determine the gauge group content and whether $\eta_{1}, \eta_{2}$ are base-point free. The resulting enumeration of $\mathcal{B}_{3}$ 's for some specific $B_{2}$ 's matches that described in the previous section using the more general constraints described in section 4. The results of the toric analysis are listed in tables 4, 5. For each base $B_{2}$ we have indicated the number of distinct $\mathcal{B}_{3}$ 's over that base (the number of possible "twists" $T$ in the $\mathbb{P}^{1}$ bundle giving acceptable $\mathcal{B}_{3}$ 's), and the number of these $\mathcal{B}_{3}$ 's that violate the base-point free condition for one or both gauge group factors. A subset of the F-theory bases $\mathcal{B}_{3}$ tabulated in table 4 have been explored in previous work; in particular, the $\mathbb{P}^{1}$ bundles over $\mathbb{P}^{2}$ and $\mathbb{F}_{m}$ described in the first four lines of the table, and the 18 toric Fano varieties were also described in [30, 137, 147].

One interesting observation from the data in table 4 is that more than half of the possible toric F-theory geometries violate the base-point free condition on at least one 


\begin{tabular}{|lr|c|c|c|c|c|}
\hline \multicolumn{2}{|l|}{ base $B_{2}$} & $h_{1,1}$ & $\# \mathcal{B}_{3}$ 's & NB $(1)$ & NB $(2)$ & $\#$ cod 3 \\
\hline$(1,1,1)$ & $\left(\mathbb{P}^{2}\right)$ & 1 & 14 & 0 & 0 & 0 \\
\hline$(0,0,0,0)$ & $\left(\mathbb{F}_{0}\right)$ & 2 & 82 & 0 & 0 & 0 \\
$(1,0,-1,0)$ & $\left(\mathbb{F}_{1}\right)$ & 2 & 109 & 0 & 0 & 0 \\
$(2,0,-2,0)$ & $\left(\mathbb{F}_{2}\right)$ & 2 & 24 & 14 & 0 & 0 \\
\hline$(0,0,-1,-1,-1)$ & $\left(d P_{2}\right)$ & 3 & 472 & 0 & 0 & 0 \\
$(1,-1,-1,-2,0)$ & 3 & 173 & 100 & 0 & 0 \\
\hline$(-1,-1,-1,-1,-1,-1)$ & $\left(d P_{3}\right)$ & 4 & 776 & 0 & 0 & 0 \\
$(0,-1,-1,-2,-1,-1)$ & 4 & 729 & 396 & 0 & 0 \\
$(0,0,-2,-1,-2,-1)$ & 4 & 312 & 213 & 42 & 0 \\
$(1,0,-2,-2,-1,-2)$ & 4 & 62 & 31 & 25 & 32 \\
\hline$(-1,-1,-2,-1,-2,-1,-1)$ & 5 & 1119 & 755 & 140 & 0 \\
$(0,-1,-1,-2,-2,-1,-2)$ & 5 & 406 & 219 & 150 & 217 \\
\hline$(-1,-1,-2,-1,-2,-2,-1,-2)$ & 6 & 351 & 149 & 185 & 173 \\
$(-1,-2,-1,-2,-1,-2,-1,-2)$ & 6 & 214 & 119 & 69 & 0 \\
$(0,-2,-1,-2,-2,-2,-1,-2)$ & 6 & 83 & 18 & 59 & 45 \\
\hline$(-1,-2,-2,-1,-2,-2,-1,-2,-2)$ & 7 & 36 & 8 & 26 & 29 \\
\hline total & & 4962 & 2022 & 696 & 496 \\
\hline
\end{tabular}

Table 4. Table of all smooth F-theory bases with smooth heterotic duals that are $\mathbb{P}^{1}$ bundles over toric bases $B_{2}$. The base $B_{2}$ is characterized by the sequence of self-intersections of toric divisors. NB (Non-Base point free) indicates the number of bases $\mathcal{B}_{3}$ that violate the base-point free condition on one (1) or both (2) sides $\Sigma_{ \pm}$. The final column is the number of models that have a toric codimension 3 locus where $f, g$ vanish to degrees 4,6 .

of the gauge factors, so that more than half of the corresponding heterotic models have generic bundles with exceptional or $\mathrm{SO}(8)$ structure group. The only models that can violate the base-point free condition are those with generalized del Pezzo bases having -2 curves, and for such bases a very high fraction of models violate the base-point free condition on at least one side. Since the vast majority of the hundreds of possible non-toric bases $B_{2}$ compatible with a smooth heterotic dual are generalized del Pezzo's, we expect that the fraction of all models with smooth heterotic duals that have exceptional or $\mathrm{SO}(8)$ structure group is quite high.

In table 5 we tabulate the number of models in the full set that have each of the possible distinct gauge algebras $\mathcal{G}_{1} \oplus \mathcal{G}_{2}$. Note that this gauge algebra represents the minimal (most generic) gauge algebra for each base. For each $\mathcal{B}_{3}$, tuning Weierstrass monomials can lead to enhanced gauge groups through "unHiggsing," corresponding on the heterotic side to special loci in bundle moduli space where the structure group $H$ becomes smaller and $G$ correspondingly larger. The minimal gauge algebra summands $\mathcal{G}_{i}$ for each base $\mathcal{B}_{3}$ 


\begin{tabular}{|r|c|c|c|c|c|c|c|c|c|}
\hline$\times$ & $\cdot$ & $\mathfrak{s u}_{2}$ & $\mathfrak{s u}_{3}$ & $\mathfrak{g}_{2}$ & $\mathfrak{s o}_{8}$ & $\mathfrak{f}_{4}$ & $\mathfrak{e}_{6}$ & $\mathfrak{e}_{7}$ & $\mathfrak{e}_{8}$ \\
\hline$\cdot$ & 712 & & & & & & & & \\
$\mathfrak{s u}_{2}$ & 499 & 47 & & & & & & & \\
$\mathfrak{s u}_{3}$ & 121 & 11 & 2 & & & & & & \\
$\mathfrak{g}_{2}$ & 589 & 62 & 7 & 34 & & & & & \\
$\mathfrak{s o}_{8}$ & 276 & 14 & 1 & 12 & 3 & & & & \\
$\mathfrak{f}_{4}$ & 1245 & 74 & 6 & 54 & 9 & 32 & & & \\
$\mathfrak{e}_{6}$ & 184 & 2 & 0 & 2 & 0 & 2 & 0 & & \\
$\mathfrak{e}_{7}$ & 890 & 24 & 0 & 14 & 2 & 13 & 0 & 4 & \\
$\mathfrak{e}_{8}$ & 15 & 0 & 0 & 0 & 0 & 0 & 0 & 0 & 0 \\
\hline
\end{tabular}

Table 5. Gauge algebras $\mathcal{G}_{1} \oplus \mathcal{G}_{2}$ arising in generic models for the $4962 \mathrm{~F}$-theory bases $\mathcal{B}_{3}$ with toric $B_{2}$ and smooth heterotic duals. Note that for many bases $\mathcal{B}_{3}$ a variety of distinct models with enhanced gauge groups can be realized when moduli are tuned to specific loci, corresponding to distinct elliptically fibered fourfolds in the F-theory picture.

are determined from the dual monomials in $f, g$ in the toric picture using table 1 . In places where the degrees of vanishing of $f, g$ do not uniquely determine the gauge algebra type, the gauge algebra is fixed by the monodromy around the codimension one divisor, which can be read off from the structure of the monomials following the discussion in section 3.1. When the vanishing degrees of $f, g, \Delta$ are $2,2,4$, the gauge algebra is $\mathfrak{s u}_{2}$ unless $g=g_{2}(u, v) z^{2}+\mathcal{O}\left(z^{3}\right)$ with $g_{2}(u, v)$ a perfect square, where $z=0$ on the divisor locus in question and $u, v$ are coordinates on the divisor. When there is no restriction other than the vanishing of certain monomials, $g_{2}(u, v)$ is only guaranteed to be a perfect square if it contains only a single even monomial $u^{2 n} v^{2 m}$. Similarly, when $f, g, \Delta$ vanish to degrees $3,4,8$, the gauge algebra factor is only $\mathfrak{e}_{6}$ when the leading part of $g$ is a perfect square, which again is only possible when it is a single even monomial. For vanishing degrees $2,3,6$ the story is slightly more subtle, but again easy to analyze in terms of the monomials. The generic gauge algebra factor is $\mathfrak{g}_{2}$. The algebra becomes $\mathfrak{s o}_{8}$ when $f_{2}^{3}=c g_{3}^{2}$, with $c$ an overall constant (complex) coefficient, which is only possible when each contains only a single monomial $f_{2}=a u^{3 n} v^{3 m}, g_{3}=b u^{2 n} v^{2 m}$. In principle, the gauge algebra could be $\mathfrak{s o}_{7}$, which can occur when $g_{3}=0$ and $f_{2}(u, v)$ is not a perfect square, but this does not occur for any of the $3 \mathrm{D}$ bases $\mathcal{B}_{3}$ considered here. (Note, however, that for more general bases that do not have smooth heterotic duals we do expect $\mathfrak{s o}_{7}$ to arise as a generic gauge algebra component. This occurs, for example, in 6D models when the base $B_{2}$ contains intersecting curves of self-intersection $-2,-3,-2$, which support the gauge algebra $\mathfrak{s u}_{2} \oplus \mathfrak{s o}_{7} \oplus \mathfrak{\mathfrak { s u } _ { 2 }}$ [8].)

In table 5 we see that the great majority of models (85\%) have some gauge group automatically imposed from the geometry, which cannot be removed by Higgsing charged moduli fields. Furthermore, most of the models either have gauge factors that are not subgroups of $\mathrm{SU}(5)$, or contain $\mathrm{SU}(2)$ or $\mathrm{SU}(3)$ factors that cannot be enhanced to $\mathrm{SU}(5)$ as discussed in the previous sections. Note that the gauge group described here is purely 
that determined by the geometry. As discussed in section 6.5, in some situations the gauge group may be modified when G-flux is taken into account. We leave further investigation of this effect to future work.

Note that there are many toric $\mathcal{B}_{3}$ 's that are $\mathbb{P}^{1}$ bundles over toric $B_{2}$ bases for which gauge algebra summands $\mathfrak{e}_{8}$ arise that are not included in this tabulation because there are codimension two curves in $\mathcal{B}_{3}$ living in the $\mathfrak{e}_{8}$ locus where the degree of vanishing of $f, g$ reaches 4,6 . These are a special class of examples of situations where a curve in the base $\mathcal{B}_{3}$ must be blown up to have a base $\mathcal{B}_{3}^{\prime}$ that can act as the base of an elliptically fibered Calabi-Yau fourfold. After this blow-up, the F-theory model no longer has a smooth heterotic dual and is not included in this analysis. In this situation the blown up base is also generically non-toric. These cases are closely analogous to base surfaces $B_{2}$ for $6 \mathrm{D}$ F-theory models that contain $-9,-10$, and -11 curves; along such curves there is an $\mathfrak{e}_{8}$ gauge algebra summand and 3,2 , or 1 points where $f, g$ vanish to degrees 4,6 and the base must be blown up for a smooth F-theory model [8, 60, 61].

Several unusual features arise in many of the 4962 models we have constructed. As mentioned in section 4.1, in some models that are otherwise well-behaved there are codimension three singularities of order $(4,6)$. It is not known whether these singularities herald a sickness of the associated 4D supergravity theories [89]; as discussed in section 7.8, such codimension three singularities may also be associated with chiral matter, G-flux, or abelian gauge symmetries. In some cases, a codimension three $(4,6)$ singularity arises at a toric point given by the intersection of three toric divisors. There are a total of 496 models with this feature (or bug) in the toric set; we have tabulated the number of threefolds $\mathcal{B}_{3}$ where this occurs for each base surface $B_{2}$ in the last column of table 4 . One of the simplest examples of a threefold with this property is the $\mathbb{P}^{1}$ bundle over the 10 th base $B_{2}$ in table 4 , characterized by divisors in the base with self-intersections $(1,0,-2,-2,-1,-2)$ and a twist divisor $T=D_{5}$, where $D_{5}$ is the divisor in $B_{2}$ with self-intersection -1 . This model has no codimension one singularities associated with nonabelian gauge groups, but $f$ and $g$ vanish to degrees $(4,6)$ at the point $\Sigma_{-} \cap D_{3} \cap D_{4}$. Note that, as mentioned in section 4.1.6 there can also be codimension three $(4,6)$ singularities arising at non-toric points, such as generically occurs on curves where $f, g$ vanish to degrees $(4,5)$. We have not attempted to classify the models with such singularities here.

Another feature that can arise is a codimension two singularity on a curve that does not lie on any divisor carrying a gauge group. While in general codimension two singularities indicate matter charged under the nonabelian gauge groups of the corresponding divisors, in this situation this interpretation is not possible. It is possible that these singularities are simply cusps in the discriminant locus with no physical meaning, or they may herald the presence of abelian U(1) factors. This occurs in roughly half (2495 of the 4962 total) of the threefold bases $\mathcal{B}_{3}$. The simplest example is the $\mathbb{P}^{1}$ bundle over $\mathbb{F}_{1}$ with twist $T=2 F$; this base gives no codimension one singularity associated with nonabelian gauge groups, but has a $(2,3)$ vanishing of $f, g$ on $S \cap \Sigma_{-}$. We leave further investigation of the models with these features to future work. 


\section{Conclusions and open questions}

In this paper we have given a global characterization of a broad class of $4 \mathrm{D} \mathcal{N}=1$ string vacua that admit both a heterotic description and a dual F-theory description. The class of vacua we have considered are described in the heterotic theory through compactification on a smooth Calabi-Yau threefold that is elliptically fibered with section over a base $B_{2}$ and carries a smooth vector bundle (and in some cases, 5-branes wrapping the elliptic fiber), and in F-theory through compactification on an elliptic fibration over a base threefold that is itself a $\mathbb{P}^{1}$ bundle with section over the same base $B_{2}$. We have shown that the number of topologically distinct vacua in this class is finite, and we have explicitly enumerated all models where the base $B_{2}$ is toric.

By focusing on the underlying geometrical and topological structure of the theories, we have developed tools and identified features of these models that do not depend on specific limits or bundle constructions on the heterotic side of the duality. We have identified from the F-theory side a simple set of constraints that are necessary and sufficient for the existence of a Calabi-Yau compactification geometry; these constraints are expressed in terms of the "twist" defining the $\mathbb{P}^{1}$ bundle on the F-theory side and related components of the second Chern class of the bundles on the heterotic side. These constraints give a detailed characterization of the circumstances under which slope-stable bundles with general structure groups should exist both for heterotic $E_{8} \times E_{8}$ and $\mathrm{SO}(32)$ theories on smooth Calabi-Yau threefolds. The structure of chiral matter on the heterotic side has implications for the interplay between chiral matter and G-flux on the F-theory side.

The results described in this work represent a small step towards a systematic characterization of the broader class of $\mathcal{N}=14 \mathrm{D}$ supergravity theories that can be realized in string theory. There are clearly many directions in which this work could be expanded further. Many more detailed aspects of the physics of the large class of models described here can be explored further using the tools described here. This work also provides a basis for a further systematic expansion of our understanding of heterotic/F-theory duality, as well as tools for expanding the range of applicability of both heterotic and F-theory approaches to string compactification. We conclude with a brief summary of some of these possible future directions.

\subsection{Detailed physics of smooth heterotic/F-theory dual pairs}

In this work, following [9], we used topological structure, in the form of axion-curvature squared terms in the 4D supergravity theory, to identify dual heterotic and F-theory geometries. This gives an association between F-theory constructions and heterotic bundles that is independent of the stable degeneration limit [19] in which the duality has been most thoroughly studied; the approach taken here has enabled a systematic classification of all smooth dual geometries where the F-theory model is described in terms of a threefold base $\mathcal{B}_{3}$ that is a $\mathbb{P}^{1}$ bundle over a base $B_{2}$. Within this class of smooth dual geometries, there are many questions that could be explored further.

The roughly 5000 models where $B_{2}$ is toric provide an extensive dataset of dual heterotic/F-theory constructions that may be useful in a variety of contexts. To aid further 
development in this direction, we have provided the details of this set of F-theory compactification geometries in a file that can be downloaded. ${ }^{24}$ This file contains a listing for each of the bases $B_{2}$ of the complete set of allowed twists $T$ describing a $\mathbb{P}^{1}$ bundle over that $B_{2}$ that does not have $(4,6)$ codimension one or two singularities, as well as the generic gauge algebras $\mathcal{G}_{1} \oplus \mathcal{G}_{2}$ generated by for codimension one singularities over that base ( $\mathrm{SO}(8)$ in those cases that have an $\mathrm{SO}(32)$ heterotic dual). The methods of this paper can be applied more generally for any of the several hundred generalized del Pezzo bases that support a smooth elliptically fibered Calabi-Yau threefold. One natural extension of the work here would be the explicit construction and classification of this broader class of (generically non-toric) bases, along the lines of the example $\mathrm{dP}_{4}$ worked out in section 7.6 (which by itself already gives rise to roughly 7000 additional $\mathcal{B}_{3}^{\prime} s$; other non-toric generalized del Pezzo surfaces are expected to similarly generate large numbers of additional examples).

For the models considered and enumerated here, many more detailed questions remain to be addressed. For each of the $\sim 5000$ toric $\mathcal{B}_{3}$ 's, there are many branches of the moduli space in which the generic gauge group is enhanced by "unHiggsing", corresponding to a tuning of Weierstrass moduli in the F-theory picture and special loci in bundle moduli space on the heterotic side. Many general aspects of the branching structure of these moduli spaces remain to be investigated. We have identified from the F-theory side specific conditions under which the dual heterotic model should admit a bundle with exceptional structure group; in many of these cases there is no explicit mathematical construction known for such models, finding such constructions represents another class of open problems related to this work. And, as mentioned throughout the text, we have not incorporated the effects of G-flux on the F-theory side; this mechanism will in general lift many geometric F-theory moduli and produce chiral matter. We hope that the explicit correspondence we have developed here will help in elucidating these issues further.

Although we have focused in this paper on general aspects of heterotic and F-theory constructions that are independent of specific models, some lessons have emerged that may be relevant for more phenomenological "model building". One general lesson from the systematic study of F-theory models both in $6 \mathrm{D}$ and in $4 \mathrm{D}$, illustrated particularly clearly in six dimensions $[60,61]$, is that a large fraction of the elliptically fibered Calabi-Yau manifolds that can be used to compactify F-theory give rise to large "non-Higgsable" gauge groups. While a clear understanding of the connection between geometrically non-Higgsable gauge groups and $4 \mathrm{D}$ physics requires a better incorporation of the effects of G-flux, the models we have studied here are among those in which the minimal geometric gauge groups are smallest, and may provide the most promising candidates for realistic models of physics. In terms of potentially phenomenologically relevant gauge groups, we have found that many $\mathrm{F}$ theory geometries contain geometric $\mathrm{SU}(2)$ and/or $\mathrm{SU}(3)$ factors that cannot be enhanced to $\mathrm{SU}(5)$, but that can for example be enhanced to $\mathrm{SO}(10), E_{6}$, or $E_{7}$. Much work has been done in constructing phenomenologically oriented F-theory models based on an SU(5) unification structure (see [149-151] for a review of some of this work, and [34, 152-158] for

\footnotetext{
${ }^{24}$ The list of 4962 toric bases $\mathcal{B}_{3}$ and associated minimal gauge groups described in section 9 is available online in an ancillary mathematica format text file at [148] or at http//ctp.lns.mit.edu/wati/data/het-Fdual-bases.m.
} 
some specific global GUT models). It would be interesting to study more broadly how the generic gauge group structures that we have explored here might play into more general model building approaches, perhaps in the context of GUT groups other than SU(5). As we have focused on generic geometric structure in the models studied here, we have also not investigated the tuning of abelian gauge group factors. Much recent work [82, 159174] has focused on the role of global U(1) factors in F-theory models. In most cases such $\mathrm{U}(1)$ factors arise only at very special tuned loci in the Weierstrass moduli space over any given F-theory base; it was recently found, however, that U(1) factors can be generic over certain special F-theory bases in 6D [61], including bases related to non-toric generalized del Pezzo bases considered here. Further investigation of U(1) factors in the class of dual heterotic/F-theory models provides another interesting direction for further work.

At present, the explicit heterotic/F-theory dual "dictionary" has been most fully determined in a corner of moduli space in which the heterotic bundles can be described via spectral (more generally cameral) covers [19, 41, 42, 80]. However, the results of this work shows that many consistent, perturbative heterotic theories cannot be described by a naive application of these constructions. For example, the bundle with $\eta=0$ of (7.34) can clearly not be described as an ordinary, smooth spectral cover (see section 7.9 for a discussion). Other specific examples arise in the class of models we have analyzed here. For instance, considering the set of 4962 toric dual pairs described in section 9, there appear to be many good F-theory geometries for which we cannot directly construct the heterotic dual bundles with standard tools, even when the heterotic structure bundle is $\mathrm{SU}(N)$. For example, of the 9474 -folds with a generic $E_{7}$ symmetry (on at least one patch), 897 of these fail to satisfy the parity condition (5.29) for $\mathrm{SU}(2)$ bundles constructed as generic, irreducible spectral covers. This indicates that if good heterotic duals exist they must either a) not be constructible as ordinary $\mathrm{SU}(2)$ spectral covers (i.e., they possess one of the limitations described in section 5.3.3) or b) the bundle moduli space contains no irreducible spectral covers at all (see [103] for example, for more exotic possibilities). It is also possible that this parity condition may be a more general constraint and may indicate some problem with the associated F-theory models, for example that may indicate a conflict with the existence of a consistent choice of G-flux. In any case, there remains something to be understood in the explicit moduli mapping of the heterotic/F-theory dual pair which could yield important new insights into both theories.

As mentioned above, we have not considered here the effects of G-flux in modifying the underlying Calabi-Yau geometry of F-theory. One of the most interesting aspects of 4dimensional heterotic/F-theory duality is the fact that deformations that change the gauge symmetry (i.e., deformations of the complex structure of $Y_{4}$ or of the bundles $V_{i}$ on $X_{3}$ ) can be obstructed. These obstructions can appear both through D- and F-term contributions to the potential in the low energy theory. A better understanding of this potential would have impact not only on the problem of moduli stabilization in heterotic/F-theory effective theories but could also lead to novel dynamical effects in the 4-dimensional theories - including, for example, the obstruction of tensionless string/small-instanton transitions and possible duality to non-commutative $D 3$ branes. We hope to explore these topics in future work. 


\subsection{Expanding heterotic/F-theory duality}

In this work we have focused on the simplest class of heterotic/F-theory dualities, where both sides have elliptically fibered Calabi-Yau geometries with a single section, and the F-theory base $\mathcal{B}_{3}$ is a $\mathbb{P}^{1}$ bundle that also has a single section. Since we expect that the heterotic and F-theory constructions are simply different mathematical approaches to describing the same physical theory in distinct limits, we expect that it should be possible to extend this duality to a much broader class of vacua, possibly at the expense of needing to introduce more complicated mathematical objects in the theory on one or both sides.

One clear question is the extent to which heterotic/F-theory duality can be systematically described when the heterotic Calabi-Yau geometry and/or bundle structure becomes singular, leading mathematically to a description in terms of more singular objects such as sheaves. While many examples of this have been studied in the literature, the appearance of structure such as enhanced gauge groups and additional geometric moduli arising through tensionless string transitions is more transparent geometrically from the point of view of Weierstrass models on the F-theory side. The framework developed here may provide a useful context in which to systematically extend the duality in these directions. The simple geometric framework of F-theory has the potential to clarify some of the mathematical questions that are rather subtle in this context on the heterotic side.

More generally, there are classes of geometries that are slightly more general than those considered here in which heterotic/F-theory duality is not understood. These include bases $\mathcal{B}_{3}$ built as more general $\mathbb{P}^{1}$ fibrations (rather than $\mathbb{P}^{1}$ bundles) and situations where either the heterotic or F-theory elliptic fibrations have multiple sections (higher rank Mordell-Weil group) or no section at all. In particular, for Calabi-Yau geometries that are elliptically fibered but have more than one section, or a multi-section, the story is not yet completely clear. F-theory models with multiple sections (higher rank Mordell-Weil group) are understood simply as models with additional U(1) factors, which generally should have natural heterotic duals. F-theory models with a multi-section but no global section have recently been incorporated into the global moduli space of Weierstrass models [81, 82]. It is less clear, however, how to construct an F-theory dual for a heterotic model on a CalabiYau threefold with multiple sections or a multi-section. While in principle such threefolds can be realized as special limits in the Weierstrass moduli space of elliptic fibrations (using the Jacobian fibration associated with threefolds having a multi-section, as in [82]), which should give a corresponding construction on the F-theory side, the details of the physics of this correspondence have not been worked out. In this paper we considered only cases where the F-theory threefold base $\mathcal{B}_{3}$ is itself a $P^{1}$ bundle with section. It is also possible to consider situations where $\mathcal{B}_{3}$ is a $P^{1}$ fibration without a section or indeed even more general geometries [66, 175].

Another example of a situation where heterotic/F-theory duality is not well understood comes from the fact that for Calabi-Yau threefolds, the moduli space of bundles $\mathcal{M}$ can have multiple components (see appendix B). If is not known how F-theory duals to such situations can be understood. A natural hypothesis is that for each component of the heterotic moduli space there would exist topologically identical, non-diffeomorphic Calabi-Yau 4-folds on the F-theory side. This is another interesting avenue for further investigation. 
Finally, on the heterotic side there are compactifications on Calabi-Yau threefolds that are not elliptically fibered. Such geometries can be reached by nonperturbative transitions from elliptically fibered Calabi-Yau threefolds, so should in principle be connected to the underlying geometric moduli space of F-theory compactifications. At present, there is no known mechanism by which F-theory can include such vacua. F-theory is at present still an incomplete physical theory, however; there is no direct action principle for the theory that incorporates all degrees of freedom. In an optimistic scenario, further development of heterotic/F-theory duality may provide some insight into a more complete formulation of the theory and a broader and more unified characterization of the full space of $\mathcal{N}=14 \mathrm{D}$ supersymmetric string theory vacua.

\section{Acknowledgments}

We would like to thank Ron Donagi, Antonella Grassi, James Gray, Thomas Grimm, Jonathan Heckman, Samuel Johnson, Denis Klevers, Gabriella Martini, David Morrison, Daniel Park, and Lucia Swanson for helpful discussions. This research was supported by the DOE under contract \#DE-FC02-94ER40818, and was also supported in part by the National Science Foundation under Grant No. PHY-1066293. We would like to thank the Simons Center for Geometry and Physics (LA and WT), the Aspen Center for Physics (WT), and the Center for the Theoretical Physics at MIT (LA) for hospitality during part of this work.

\section{A Properties of elliptically fibered Calabi-Yau three- and fourfolds}

In this appendix we briefly review a collection of useful results regarding the geometry and topology of elliptically fibered Calabi-Yau manifolds (see [19, 30] for a more complete treatment). We focus on smooth elliptically fibered Calabi-Yau threefolds, $\pi: X_{3} \rightarrow B_{2}$ with a single section (which defines $B_{2}$ as an algebraic sub-manifold within $X_{3}$ ). If $X_{3}$ is in Weierstrass form, a minimal set of divisors ${ }^{25}$ that span the Picard group of $X_{3}$ is given by the zero section, $D_{0}$ and divisors pulled back from the base of the form $D_{\alpha}=\pi^{*}\left(D_{\alpha}^{\text {base }}\right)$, where $D_{\alpha}^{\text {base }}, \alpha=1, \ldots h^{1,1}\left(B_{2}\right)$ is an ample divisor on $B_{2}$. For such smooth, minimal elliptic fibrations, $h^{1,1}\left(X_{3}\right)=h^{1,1}\left(B_{2}\right)+1$. We will denote the basis of $\{1,1\}$-forms dual to the divisors above as $\left\{\omega_{0}, \omega_{\alpha}\right\}$.

By virtue of this simple fibration structure, the triple intersection numbers of these divisors exhibit a universal behavior. First, since the base is a 2 -fold it is clear that

$$
D_{\alpha} \cap D_{\beta} \cap D_{\gamma}=0
$$

Moreover, from the very definition of what it means for $D_{0}$ to be section (and not a multisection) it is guaranteed that for any two-form $\zeta$ on $B_{2}$ (dual to a single point),

\footnotetext{
${ }^{25}$ We focus here on a minimal form of elliptically fibered threefold in which all exceptional curves in the fiber have been blown down.
} 
$D_{0} \cap \zeta=1$ (that is, the zero section intersects each elliptic fiber precisely once). It follows from this fact that

$$
D_{0} \cap D_{\alpha} \cap D_{\beta}=m_{\alpha \beta}
$$

where $m_{\alpha \beta}=D_{\alpha}^{\text {base }} \cap D_{\beta}^{\text {base }}$. These facts are enough to derive the following important cohomological identity on $\{2,2\}$ forms,

$$
\omega_{0} \wedge \omega_{0}=K \wedge \omega_{0}
$$

where $K$ is the canonical class of the base, $K=-c_{1}\left(B_{2}\right)=K^{\alpha} \omega_{\alpha}$. With these results, the triple intersection numbers of $X_{3}$

$$
d_{A B C}=\int_{X_{3}} \omega_{A} \wedge \omega_{B} \wedge \omega_{C}
$$

where $\omega_{A}=\left\{\omega_{0}, \omega_{\alpha}\right\}$, are given by

$$
\begin{array}{ll}
d_{000}=m_{\alpha \beta} K^{\alpha} K^{\beta} & d_{00 \alpha}=m_{\alpha \beta} K^{\beta} \\
d_{0 \alpha \beta}=m_{\alpha \beta} & d_{\alpha \beta \gamma}=0
\end{array}
$$

With these intersection numbers and a chosen Kähler form $\omega=t^{0} \omega_{0}+t^{\alpha} \omega_{\alpha}$, the volume of $X_{3}$ takes the form

$$
\operatorname{Vol}\left(X_{3}\right)=\frac{1}{3 !} \int_{X} \omega \wedge \omega \wedge \omega=\frac{1}{3 !}\left(d_{000}\left(t^{0}\right)^{3}+3 d_{00 \alpha}\left(t^{0}\right)^{2} t^{\alpha}+3 d_{0 \alpha \beta} t^{0} t^{\alpha} t^{\beta}\right)
$$

The fibration structure guarantees that the second Chern class of $X_{3}$ can be written as $[19]$

$$
c_{2}\left(T X_{3}\right)=12 c_{1}\left(B_{2}\right) \wedge \omega_{0}+c_{2}\left(B_{2}\right)+11 c_{1}\left(B_{2}\right)^{2}
$$

where in addition the topology of $B_{2}$ satisfies

$$
\chi\left(B_{2}\right)=\int_{B_{2}} c_{2}\left(B_{2}\right)=2+h^{1,1}\left(B_{2}\right) \quad, \quad \int_{B_{2}} c_{1}^{2}\left(B_{2}\right)=K^{\alpha} K^{\beta} m_{\alpha \beta}=10-h^{1,1}\left(B_{2}\right)
$$

Finally, using the redundancy relation on $\{2,2\}$ forms in (A.3) it is possible to write the second Chern class of any bundle, $V$, on $X_{3}$

$$
c_{2}(V)=\eta \wedge \omega_{0}+\zeta
$$

where $\eta$ and $\zeta$ are pullbacks through $\pi$ of $\{1,1\}\{2,2\}$ forms from $B_{2}$.

\section{B A brief exploration of rigid bundles}

A novel feature of four-dimensional compactifications of heterotic string theory/F-theory is the possibility of multiple components in the dual (vector bundle/fourfold) moduli spaces. In the case of heterotic/F-theory duality, such multiple components to the moduli space have not yet been studied in detail. Indeed, thus far in the literature the correspondence 
between vector bundle moduli spaces in heterotic theories and the complex structure moduli space of Calabi-Yau fourfolds has only been studied in the case of single, connected components [19].

For the moduli space of stable sheaves on Calabi-Yau threefolds a natural case of interest is given when the local moduli space in fact contains an isolated, rigid component. In general, given two bundles with the same topology, it is difficult to decide whether or not they reside in the same component of a global bundle moduli space. However, if one bundle is rigid (and the other has local deformation moduli), it is clear that the rigid bundle consists of its own distinct component to bundle moduli space. As a result, a search for rigid bundles is one of the simplest probes for multiple components of bundle moduli spaces.

For elliptically fibered Calabi-Yau threefolds, it is straightforward to come by examples of such rigid vector bundles. For instance, over the Calabi-Yau threefold defined as a single degree $\{3,3\}$ hypersurface in the product, $\mathbb{P}^{2} \times \mathbb{P}^{2}$, of two projective spaces, consider the following poly-stable $\mathrm{SU}(2)$ bundle

$$
V_{2}=L+L^{\vee}=\mathcal{O}\left(-H_{1}+H_{2}\right)+\mathcal{O}\left(H_{1}-H_{2}\right)
$$

where $H_{1}, H_{2}$ are the restrictions of the hyperplanes of each ambient $\mathbb{P}^{2}$ factor to $X_{3}$. On this space, $V_{2}$ is rigid, since the dimension of the space of bundle-valued singlets is given by

$$
h^{1}\left(X_{3}, L^{\otimes 2}\right)=h^{1}\left(X_{3}, L^{\vee \otimes 2}\right)=0
$$

for generic values of the complex structure of $X_{3}$ [99]. Geometrically the spaces $H^{1}\left(X_{3}, L^{\otimes 2}\right), H^{1}\left(X_{3}, L^{\vee \otimes 2}\right)$ constitute the space of non-trivial extensions (for example $H^{1}\left(X_{3}, L^{\otimes 2}\right)$ parameterizes the space of non-trivial extensions $0 \rightarrow L \rightarrow V_{2} \rightarrow L^{\vee} \rightarrow 0$ ) which parameterize how $L, L^{\vee}$ may be non-trivially "glued" back into an indecomposable $\mathrm{SU}(2)$ bundle. Since the space of such extensions vanishes in these cases, the split bundle $L+L^{\vee}$ has no infinitesimal deformations, that is, it is rigid.

Although the example above is interesting from the point of view of vector bundle moduli spaces, it is not clear what the impact of such examples will be in the heterotic-Ftheory pairs constructed in this work. Although the threefold above is elliptically fibered, it has no section and cannot be written in Weierstrass form. At present its F-theory dual (if any) is unknown.

In this appendix, we make a tentative exploration of whether it is possible to obtain isolated components to the moduli space of $\mathrm{SU}(2)$ bundles, such as the one described above, over the class of Calabi-Yau threefolds considered here - that is, elliptically fibered threefolds with a single section, obeying the topological identities listed in appendix A. Once again, we can search for bundles of the form

$$
L+L^{\vee}
$$

where $L$ is a holomorphic line bundle on $X_{3}$ satisfying $\mu(L)=0$ in the Kähler cone, as required for supersymmetry by (5.6). This reducible bundle in (B.3) will be rigid if $h^{1}\left(X, L^{\otimes 2}\right)=h^{1}\left(X,\left(L^{\vee}\right)^{\otimes 2}\right)=0$ 
As we will argue below, however, for the geometry in consideration in this work, such examples appear to be rare and we have obtained no explicit examples. This result is fully consistent with the fact that in the dual F-theory geometry we find a single Calabi-Yau fourfold (with a single connected (and non-trivial) component to its complex structure moduli space) for each choice of vector bundle topology/twisting parameter, $T$.

To see this, we must consider the line bundle cohomology of $L^{\otimes 2}$ on $\pi: X_{3} \rightarrow B_{2}$. Here we are aided by the formalism of Leray Spectral sequences [98]. According to this spectral sequence for the fibration $\pi: X_{3} \rightarrow B_{2}$, we have a natural bi-grading such that for any bundle $V$ on $X_{3}$,

$$
H^{p}(X, V)=\sum_{p=l+m} E_{\infty}^{l, m}
$$

where

$$
E_{1}^{l, m}=H^{l}\left(B_{2}, R^{m} \pi_{*}(V)\right)
$$

and and $R^{m} \pi_{*}(V)$ is the $m$-th direct image sheaf of the bundle $V$ (pushed forward under the fibration $\pi$ ). We need not concern ourselves with the iteration of the sequence via the maps $d_{r}: E_{r}^{p, q} \rightarrow E_{r}{ }^{p+r, q-r+1}$, since the spectral sequence terminates at $E_{1}$. To see this, note that on any open set $\mathcal{U}$ on $\mathbb{P}^{1}$, the $m$-th direct image sheaf, $R^{m} \pi_{*}(V)$ can be locally represented by the pre-sheaf

$$
\mathcal{U} \rightarrow H^{m}\left(f^{-1}(\mathcal{U}), R^{m} \pi_{*}(V)\right)
$$

For elliptic fibrations, however, the fiber (locally isomorphic to $f^{-1}(\mathcal{U})$ ) is one dimensional. As a result, $R^{m} \pi_{*}(V)$ is non-vanishing only for $m=0,1$ and the spectral sequence terminates at

$$
E_{\infty}=E_{1}
$$

To analyze the cohomology, we further need to observe how a line bundle of the form

$$
L=\mathcal{O}\left(a \sigma+b^{\alpha} \pi^{*}\left(D_{\alpha}\right)\right)
$$

behaves under the push-forward functor. The first useful useful observation is that line bundles of the form $\mathcal{O}\left(b^{\alpha} D_{\alpha}\right)$ built from divisors pulled back from the base $B_{2}$ satisfies $\pi^{*} \mathcal{O}_{B_{2}}\left(b^{\alpha} D_{\alpha}\right)=\mathcal{O}_{X_{3}}\left(b^{\alpha} \pi^{*}\left(D_{\alpha}\right)\right)$. This implies that under push-forwards we have the so-called "projection formula". For a fibration $\pi: X_{3} \rightarrow B_{2}$, and any bundles $V$ on $X$ and $U$ on $B$,

$$
R^{q} \pi_{*}\left(V \otimes \pi^{*} U\right)=R^{q} \pi_{*}(V) \otimes U
$$

To determine the cohomology of $L=\mathcal{O}_{X_{3}}\left(a \sigma+b^{\alpha} \pi^{*}\left(D_{\alpha}\right)\right)$ then, we need only consider $R^{i} \pi_{*}\left(\mathcal{O}_{X_{3}}\left(a \sigma+b^{\alpha} \pi^{*}\left(D_{\alpha}\right)\right)=R^{i} \pi_{*}\left(\mathcal{O}_{X_{3}}(a \sigma)\right) \otimes \mathcal{O}_{B_{2}}\left(b^{\alpha} D_{\alpha}\right)\right.$. Moreover, the structure of $R^{i} \pi_{*}\left(\mathcal{O}_{X_{3}}(a \sigma)\right)$ can straightforwardly be determined by considering the Koszul sequence:

$$
\left.0 \rightarrow \mathcal{O}_{X_{3}}(-\sigma) \rightarrow \mathcal{O}_{X_{3}} \rightarrow \mathcal{O}\right|_{\sigma=B_{2}} \rightarrow 0
$$

Twisting this by $\mathcal{O}_{X_{3}}(\sigma)$ yields

$$
\left.0 \rightarrow \mathcal{O}_{X_{3}} \rightarrow \mathcal{O}_{X_{3}}(\sigma) \rightarrow \mathcal{O}(\sigma \cdot \sigma)\right|_{\sigma=B_{2}} \rightarrow 0
$$


But by (A.3) and the definition of $\sigma$ as the zero-section this is simply

$$
\left.0 \rightarrow \mathcal{O}_{X_{3}} \rightarrow \mathcal{O}_{X_{3}}(\sigma) \rightarrow \mathcal{O}\left(K_{2} \cdot \sigma\right)\right|_{\sigma=B_{2}} \rightarrow 0
$$

and pushing forward to $B_{2}$ gives the short exact sequence

$$
0 \rightarrow \mathcal{O}_{B_{2}} \rightarrow R^{0} \pi_{*}\left(\mathcal{O}_{X_{3}}(\sigma)\right) \rightarrow K_{2} \rightarrow 0
$$

For $B_{2}$ the base of a $\mathrm{CY}$ threefold, the above sequence splits and we have determined the direct image sheaf:

$$
R^{0} \pi_{*}\left(\mathcal{O}_{X_{3}}(\sigma)\right)=\mathcal{O}_{B_{2}} \oplus K_{2}
$$

The calculation outlined above can be iterated inductively to find the higher direct image sheaves $R^{i} \pi_{*}\left(\mathcal{O}_{X_{3}}(a \sigma)\right)$ for $a>1$ in a similar manner. It is straightforward to demonstrate that

$$
\begin{array}{rlrl}
R^{0} \pi_{*}\left(\mathcal{O}_{X_{3}}(2 \sigma)\right) & =\mathcal{O}_{B_{2}} \oplus K_{2} \oplus K_{2}^{\otimes 2} & & R^{1} \pi_{*}\left(\mathcal{O}_{X_{3}}(2 \sigma)\right)=0 \\
R^{0} \pi_{*}\left(\mathcal{O}_{X_{3}}(3 \sigma)\right) & =\mathcal{O}_{B_{2}} \oplus K_{2} \oplus K_{2}^{\otimes 2} \oplus K_{2}^{\otimes 3} & & R^{1} \pi_{*}\left(\mathcal{O}_{X_{3}}(3 \sigma)\right)=0 \\
\vdots & & & \vdots \\
R^{0} \pi_{*}\left(\mathcal{O}_{X_{3}}(a \sigma)\right)=\operatorname{Sym}^{a}\left(\mathcal{O}_{B_{2}} \oplus K_{2}\right) & & R^{1} \pi_{*}\left(\mathcal{O}_{X_{3}}(a \sigma)\right)=0
\end{array}
$$

Similar results for $a<0$ can be found by using Grothendieck duality: for any sheaf $F$ on $X_{3}$, the push-forward functors obey the following relation:

$$
R^{1-i} \pi_{*}\left(F^{\vee} \otimes \omega_{\mathbb{X}_{3} \mid B_{2}}\right)=\left(R^{i} \pi_{*} F\right)^{\vee} \quad, i=0,1
$$

where $\omega_{X_{3} \mid B_{2}}=K_{X_{3}} \otimes \pi^{*}\left(K_{2}^{\vee}\right)=\pi^{*}\left(K_{2}^{\vee}\right)$ is the "dualyzing sheaf" [98]. With these results in hand, we can now in principle calculate all line bundle cohomology on $X_{3}$. In order to build reducible, rigid $\mathrm{SU}(2)$ bundles like those above we must note that for the line bundles of interest, $\mu(L)=0$, and hence [99]

$$
H^{0}(X, L)=H^{3}(X, L)=0
$$

Thus, to construct a rigid $\mathrm{SU}(2)$ bundle we must use the results above for line bundle cohomology and further ask, for what values of $a, b$ in (B.8) can we have $H^{1}(X, L)=$ $H^{1}\left(X, L^{\vee}\right)=0$ ? By (B.4) and (B.5) it is clear that we require

$$
H^{1}\left(B_{2}, R^{0} \pi_{*} L\right)=H^{0}\left(B_{2}, R^{1} \pi_{*} L\right)=0
$$

in order to satisfy $H^{1}(X, L)=0$ and

$$
H^{2}\left(B_{2}, R^{0} \pi_{*} L\right)=H^{1}\left(B_{2}, R^{1} \pi_{*} L\right)=0
$$

for $H^{2}(X, L)=H^{1}\left(X, L^{\vee}\right)=0$ (by Serre duality). This, coupled with (B.20) means that the direct image sheaves $R^{0} \pi_{*} L$ and $R^{1} \pi_{*} L$ must have entirely vanishing cohomology on $B_{2}$. However, as we will see below, this does not occur for simple threefolds of the type we are considering here. 
To see that it is rare for $H^{i}\left(B_{2}, R^{0} \pi_{*} L\right)=0 \forall i$, it is useful to consider the index of $R^{0} \pi_{*} L$ using the Riemann-Roch theorem. With $L$ as in (B.8)

$$
R^{0} \pi_{*} L=\left(\mathcal{O}_{B_{2}} \oplus K_{2} \oplus \ldots K_{2}^{\otimes a}\right) \otimes \mathcal{O}_{B_{2}}\left(b^{\alpha} D_{\alpha}\right)
$$

we have that for each term in the sum, the index is additive. Thus

$$
\chi\left(R^{0} \pi_{*} L\right)=\chi\left(\mathcal{O}_{B_{2}}\left(b^{\alpha} D_{\alpha}\right)+\chi\left(K_{2} \otimes \mathcal{O}_{B_{2}}\left(b^{\alpha} D_{\alpha}\right)\right) \ldots\right.
$$

Letting $D=b^{\alpha} D_{\alpha}$ and using the fact that for any divisor, $A \subset B_{2}$,

$$
\chi(A)=1+\frac{1}{2} A \cdot\left(A-K_{2}\right)
$$

we have

$$
\chi\left(R^{0} \pi_{*} L\right)=(a+1)+D \cdot D
$$

If we demand that the index vanishes as a necessary condition for entirely vanishing cohomology, we require $D \cdot D=-(a+1)$ for some curve $D \subset B_{2}$. Putting this together with other geometric constraints in the problem, we see that the Bogomolov bound of section 5.1 places a positivity condition on $c_{2}(V)$. For $V=L \oplus L^{\vee}$ this enforces that $a \geq 0$. Finally, it can be noted that for the case $a=0$, the line bundles can be verified to have non-vanishing cohomology. Thus, here we will consider $a>0$.

For the geometries considered in this work, we have at most -2 curves, thus without loss of generality we can restrict ourselves to line bundles of the form $\mathcal{O}\left(\sigma+b^{\alpha} D_{\alpha}\right)$ where $D=b^{\alpha} D_{\alpha}$ is a -2 curve. Although there do exist curves of this type (for example the divisor $S$ in $\mathbb{F}_{2}$ ), it can be verified on a case-by-case basis that here the necessary condition is not in fact sufficient and $H^{1}\left(B_{2}, R^{0} \pi_{*} L\right) \neq 0$. Although we have not rigorously ruled out all possible -2 curves in our set of base manifolds $B_{2}$, systematic searches have found no examples with entirely vanishing cohomology. Thus, we expect that for the simple geometries outlined in appendix A, no reducible $\mathrm{SU}(2)$ bundles of the form shown in (B.3) exist as rigid components in the moduli space. It would be nice, however, to have a more general abstract proof of this result. If rigid bundles can be found within the context of heterotic/F-theory duality, it would be interesting to investigate the dual F-theory constructions. Some possibly related F-theory models may exist; for $6 \mathrm{D}$ compactifications over $B_{2}=\mathbb{P}^{2}$, some models with exotic matter were identified in [68] that are similarly rigid in the sense that they have no moduli that preserve the gauge group and matter content.

Open Access. This article is distributed under the terms of the Creative Commons Attribution License (CC-BY 4.0), which permits any use, distribution and reproduction in any medium, provided the original author(s) and source are credited.

\section{References}

[1] M.B. Green, J.H. Schwarz and E. Witten, Superstring theory, volume 1 and 2, Cambridge Univ. Pr., Cambridge U.K. (1989).

[2] J. Polchinski, String theory, volume 1 and 2, Cambridge Univ. Pr., Cambridge U.K. (1998). 
[3] L. Susskind, The anthropic landscape of string theory, hep-th/0302219 [INSPIRE].

[4] M. Graña, Flux compactifications in string theory: a comprehensive review, Phys. Rept. 423 (2006) 91 [hep-th/0509003] [INSPIRE].

[5] F. Denef, M.R. Douglas and S. Kachru, Physics of string flux compactifications, Ann. Rev. Nucl. Part. Sci. 57 (2007) 119 [hep-th/0701050] [INSPIRE].

[6] V. Kumar and W. Taylor, String universality in six dimensions, Adv. Theor. Math. Phys. 15 (2011) 325 [arXiv:0906.0987] [INSPIRE].

[7] V. Kumar, D.R. Morrison and W. Taylor, Global aspects of the space of $6 D N=1$ supergravities, JHEP 11 (2010) 118 [arXiv:1008.1062] [INSPIRE].

[8] D.R. Morrison and W. Taylor, Classifying bases for $6 D$ F-theory models, Central Eur. J. Phys. 10 (2012) 1072 [arXiv:1201.1943] [INSPIRE].

[9] T.W. Grimm and W. Taylor, Structure in $6 D$ and $4 D N=1$ supergravity theories from F-theory, JHEP 10 (2012) 105 [arXiv: 1204.3092] [INSPIRE].

[10] R. Donagi, B.A. Ovrut, T. Pantev and D. Waldram, Standard model bundles on nonsimply connected Calabi-Yau threefolds, JHEP 08 (2001) 053 [hep-th/0008008] [INSPIRE].

[11] R. Donagi, B.A. Ovrut, T. Pantev and D. Waldram, Standard model bundles, Adv. Theor. Math. Phys. 5 (2002) 563 [math/0008010] [INSPIRE].

[12] L.B. Anderson, Y.-H. He and A. Lukas, Heterotic compactification, an algorithmic approach, JHEP 07 (2007) 049 [hep-th/0702210] [INSPIRE].

[13] L.B. Anderson, Y.-H. He and A. Lukas, Monad bundles in heterotic string compactifications, JHEP 07 (2008) 104 [arXiv: 0805. 2875] [INSPIRE].

[14] L.B. Anderson, J. Gray, Y.-H. He and A. Lukas, Exploring positive monad bundles and a new heterotic standard model, JHEP 02 (2010) 054 [arXiv:0911.1569] [INSPIRE].

[15] L.B. Anderson, A. Constantin, J. Gray, A. Lukas and E. Palti, A comprehensive scan for heterotic SU(5) GUT models, JHEP 01 (2014) 047 [arXiv:1307.4787] [INSPIRE].

[16] L.B. Anderson, J. Gray, A. Lukas and E. Palti, Heterotic line bundle standard models, JHEP 06 (2012) 113 [arXiv:1202.1757] [INSPIRE].

[17] L.B. Anderson, J. Gray, A. Lukas and E. Palti, Two hundred heterotic standard models on smooth Calabi-Yau threefolds, Phys. Rev. D 84 (2011) 106005 [arXiv:1106.4804] [INSPIRE].

[18] M. Gabella, Y.-H. He and A. Lukas, An abundance of heterotic vacua, JHEP 12 (2008) 027 [arXiv:0808.2142] [INSPIRE].

[19] R. Friedman, J. Morgan and E. Witten, Vector bundles and F-theory, Commun. Math. Phys. 187 (1997) 679 [hep-th/9701162] [INSPIRE].

[20] H. Hayashi, R. Tatar, Y. Toda, T. Watari and M. Yamazaki, New aspects of heterotic/F-theory duality, Nucl. Phys. B 806 (2009) 224 [arXiv:0805.1057] [inSPIRE].

[21] D.R. Morrison and C. Vafa, Compactifications of F-theory on Calabi-Yau threefolds. 1, Nucl. Phys. B 473 (1996) 74 [hep-th/9602114] [INSPIRE].

[22] D.R. Morrison and C. Vafa, Compactifications of F-theory on Calabi-Yau threefolds. 2, Nucl. Phys. B 476 (1996) 437 [hep-th/9603161] [INSPIRE]. 
[23] M.J. Duff, R. Minasian and E. Witten, Evidence for heterotic/heterotic duality, Nucl. Phys. B 465 (1996) 413 [hep-th/9601036] [INSPIRE].

[24] N. Seiberg and E. Witten, Comments on string dynamics in six-dimensions, Nucl. Phys. B 471 (1996) 121 [hep-th/9603003] [INSPIRE].

[25] M. Gross, A finiteness theorem for elliptic Calabi-Yau threefolds, Duke Math. J. 74 (1994) 271 [alg-geom/9305002] [INSPIRE].

[26] M. Maruyama, On boundedness of torsion free sheaves, J. Math. Kyoto Univ. 21 (1981) 673.

[27] A. Langer, Semistable sheaves in positive characteristic, Ann. Math. 159 (2004) 251 [Addendum ibid. 160 (2004) 1211].

[28] M. Demazure, H.C. Pinkham and B. Teissier eds., Seminaire sur les singularités des surfaces (in French), Lect. Notes Math. 777, Springer, Germany (1980).

[29] M. Cvetič, J. Halverson, D. Klevers and P. Song, On finiteness of type IIB compactifications: magnetized branes on elliptic Calabi-Yau threefolds, JHEP 06 (2014) 138 [arXiv:1403.4943] [INSPIRE].

[30] A. Klemm, B. Lian, S.S. Roan and S.-T. Yau, Calabi-Yau fourfolds for M-theory and F-theory compactifications, Nucl. Phys. B 518 (1998) 515 [hep-th/9701023] [INSPIRE].

[31] I. Brunner and R. Schimmrigk, F-theory on Calabi-Yau fourfolds, Phys. Lett. B 387 (1996) 750 [hep-th/9606148] [INSPIRE].

[32] I. Brunner, M. Lynker and R. Schimmrigk, Unification of M-theory and F-theory Calabi-Yau fourfold vacua, Nucl. Phys. B 498 (1997) 156 [hep-th/9610195] [INSPIRE].

[33] I. Brunner, M. Lynker and R. Schimmrigk, Dualities and phase transitions for Calabi-Yau threefolds and fourfolds, Nucl. Phys. Proc. Suppl. 56B (1997) 120 [hep-th/9703182] [INSPIRE].

[34] J. Knapp, M. Kreuzer, C. Mayrhofer and N.-O. Walliser, Toric construction of global F-theory GUTs, JHEP 03 (2011) 138 [arXiv:1101.4908] [INSPIRE].

[35] J. Gray, A.S. Haupt and A. Lukas, All complete intersection Calabi-Yau four-folds, JHEP 07 (2013) 070 [arXiv: 1303.1832] [INSPIRE].

[36] J. Gray, A.S. Haupt and A. Lukas, Topological invariants and fibration structure of complete intersection Calabi-Yau four-folds, arXiv:1405.2073 [INSPIRE].

[37] G. Rajesh, Toric geometry and F-theory/heterotic duality in four-dimensions, JHEP 12 (1998) 018 [hep-th/9811240] [INSPIRE].

[38] P. Berglund and P. Mayr, Stability of vector bundles from F-theory, JHEP 12 (1999) 009 [hep-th/9904114] [INSPIRE].

[39] R. Friedman, J.W. Morgan and E. Witten, Vector bundles over elliptic fibrations, alg-geom/9709029 [INSPIRE].

[40] R.Y. Donagi, Principal bundles on elliptic fibrations, Asian J. Math 1 (1997) 214 [alg-geom/9702002] [INSPIRE].

[41] R. Donagi, Spectral covers, in Current topics in complex algebraic geometry, MSRI pub. 28 (1992) 65 [alg-geom/9505009].

[42] R. Donagi, Heterotic/F-theory duality: ICMP lecture, in Mathematical physics, Brisbane Australia (1997), pg. 206 [hep-th/9802093] [INSPIRE]. 
[43] E. Looijenga, Root systems and elliptic curves, Invent. Math. 38 (1977) 17.

[44] E. Looijenga, Invariant theory for generalized root systems, Invent. Math. 61 (1980) 1.

[45] G. Curio, Chiral matter and transitions in heterotic string models, Phys. Lett. B 435 (1998) 39 [hep-th/9803224] [inSPIRE].

[46] C. Vafa, Evidence for F-theory, Nucl. Phys. B 469 (1996) 403 [hep-th/9602022] [InSPIRE].

[47] F. Denef, Les Houches lectures on constructing string vacua, arXiv:0803.1194 [INSPIRE].

[48] A. Clingher and J.W. Morgan, Mathematics underlying the F-theory/heterotic string duality in eight-dimensions, Commun. Math. Phys. 254 (2005) 513 [math/0308106] [INSPIRE].

[49] G. Lopes Cardoso, G. Curio, D. Lüst and T. Mohaupt, On the duality between the heterotic string and F-theory in eight-dimensions, Phys. Lett. B 389 (1996) 479 [hep-th/9609111] [INSPIRE].

[50] W. Lerche and S. Stieberger, Prepotential, mirror map and F-theory on K3, Adv. Theor. Math. Phys. 2 (1998) 1105 [Erratum ibid. 3 (1999) 1199] [hep-th/9804176] [InSPIRE].

[51] J. McOrist, D.R. Morrison and S. Sethi, Geometries, non-geometries and fluxes, Adv. Theor. Math. Phys. 14 (2010) [arXiv: 1004.5447] [InSPIRE].

[52] K. Kodaira, On compact analytic surfaces. II, Ann. Math. 77 (1963) 563.

[53] K. Kodaira, On compact analytic surfaces. III, Ann. Math. 78 (1963) 1.

[54] O.J. Ganor, D.R. Morrison and N. Seiberg, Branes, Calabi-Yau spaces and toroidal compactification of the $N=1$ six-dimensional $E_{8}$ theory, Nucl. Phys. B 487 (1997) 93 [hep-th/9610251] [INSPIRE].

[55] W. Taylor, TASI lectures on supergravity and string vacua in various dimensions, arXiv:1104.2051 [INSPIRE].

[56] M. Bershadsky et al., Geometric singularities and enhanced gauge symmetries, Nucl. Phys. B 481 (1996) 215 [hep-th/9605200] [INSPIRE].

[57] W.P. Barth, K. Hulek, C.A.M. Peters and A. Van de Ven, Compact complex surfaces, Springer, Germany (2004).

[58] M. Reid, Chapters on algebraic surfaces, in Complex algebraic geometry, Park City U.S.A. (1993), IAS/Park City Math. Ser. 3 (1997) 3 [alg-geom/9602006].

[59] A. Grassi, On minimal models of elliptic threefolds, Math. Ann. 290 (1991) 287.

[60] D.R. Morrison and W. Taylor, Toric bases for 6D F-theory models, Fortsch. Phys. 60 (2012) 1187 [arXiv: 1204.0283] [INSPIRE].

[61] G. Martini and W. Taylor, 6D F-theory models and elliptically fibered Calabi-Yau threefolds over semi-toric base surfaces, arXiv:1404.6300 [INSPIRE].

[62] S. Donaldson, Anti self-dual Yang-Mills connections over complex algebraic surfaces and stable vector bundles, Proc. London Math. Soc. 3 (1985) 1.

[63] K. Uhlenbeck and S.T. Yau, On the existence of Hermitian Yang-Mills connections in stable bundles, Comm. Pure App. Math. 39 (1986) 257.

[64] K. Uhlenbeck and S.T. Yau, A note on our previous paper: on the existence of Hermitian Yang-Mills connections in stable vector bundles, Comm. Pure App. Math. 42 (1986) 703. 
[65] P.S. Aspinwall, Aspects of the hypermultiplet moduli space in string duality, JHEP 04 (1998) 019 [hep-th/9802194] [INSPIRE].

[66] R. Donagi, S. Katz and M. Wijnholt, Weak coupling, degeneration and log Calabi-Yau spaces, arXiv:1212.0553 [INSPIRE].

[67] V. Sadov, Generalized Green-Schwarz mechanism in F-theory, Phys. Lett. B 388 (1996) 45 [hep-th/9606008] [INSPIRE].

[68] V. Kumar, D.S. Park and W. Taylor, 6D supergravity without tensor multiplets, JHEP 04 (2011) 080 [arXiv: 1011.0726] [InSPIRE].

[69] D.R. Morrison and W. Taylor, Matter and singularities, JHEP 01 (2012) 022 [arXiv: 1106.3563] [INSPIRE].

[70] P.S. Aspinwall and D.R. Morrison, Point-like instantons on K3 orbifolds, Nucl. Phys. B 503 (1997) 533 [hep-th/9705104] [INSPIRE].

[71] P.S. Aspinwall and R.Y. Donagi, The heterotic string, the tangent bundle and derived categories, Adv. Theor. Math. Phys. 2 (1998) 1041 [hep-th/9806094] [INSPIRE].

[72] D. Huybrechts and M. Lehn, The geometry of moduli spaces of sheaves, Cambridge University Press, Cambridge U.K. (2010).

[73] G. Ellingsrud and L. Gottsche, Variation of moduli spaces and Donaldson invariants under change of polarization, J. reine angew. Math. 467 (1995) 1 [alg-geom/9410005].

[74] Z. Qin, Birational properties of moduli spaces of stable locally free rank-2 sheaves on algebraic surfaces, Manuscripta Math. 72 (1991) 163.

[75] W.P. Li and Z. Qin, Donaldson-Thomas invariants of certain Calabi-Yau 3-folds, arXiv: 1002.4080.

[76] W.P. Li and Z. Qin, Stable rank-2 bundles on Calabi-Yau manifolds, Internat. J. Math. 14 (2003) 1097.

[77] R. Friedman and Z. Qin, Flips of moduli spaces and transition formulas for Donaldson polynomial invariants of rational surfaces, Commun. Anal. Geom. 3 (1995) 11 [alg-geom/9410007].

[78] L.B. Anderson, J. Gray and B.A. Ovrut, Transitions in the web of heterotic vacua, Fortsch. Phys. 59 (2011) 327 [arXiv: 1012.3179] [INSPIRE].

[79] S.K. Donaldson and R.P. Thomas, Gauge theory in higher dimensions, in The geometric universe, Oxford Univ. Press, Oxford U.K. (1998), pg. 31.

[80] G. Curio and R.Y. Donagi, Moduli in $N=1$ heterotic/F-theory duality, Nucl. Phys. B 518 (1998) 603 [hep-th/9801057] [INSPIRE].

[81] V. Braun and D.R. Morrison, F-theory on genus-one fibrations, arXiv: 1401.7844 [INSPIRE].

[82] D.R. Morrison and W. Taylor, Sections, multisections and U(1) fields in F-theory, arXiv: 1404.1527 [INSPIRE].

[83] S. Katz, D.R. Morrison, S. Schäfer-Nameki and J. Sully, Tate's algorithm and F-theory, JHEP 08 (2011) 094 [arXiv:1106.3854] [InSPIRE].

[84] H.C. Pinkham, Simple elliptic singularities, Del Pezzo surfaces and Cremona transformations, Proc. Sympos. Pure Math. 30 (1977) 69, Amer. Math. Soc., U.S.A. (1977). 
[85] U. Persson, Configurations of Kodaira fibers on rational elliptic surfaces, Math. Z. 205 (1990) 1.

[86] R. Miranda, Persson's list of singular fibers for a rational elliptic surface, Math. Z. 205 (1990) 191.

[87] W. Fulton, Introduction to toric varieties, Ann. Math. Study 131, Princeton University Press, Princeton U.S.A. (1993).

[88] J. Knapp and M. Kreuzer, Toric methods in F-theory model building, Adv. High Energy Phys. 2011 (2011) 513436 [arXiv:1103.3358] [INSPIRE].

[89] P. Candelas, D.-E. Diaconescu, B. Florea, D.R. Morrison and G. Rajesh, Codimension three bundle singularities in F-theory, JHEP 06 (2002) 014 [hep-th/0009228] [INSPIRE].

[90] A. Grassi and D.R. Morrison, Group representations and the Euler characteristic of elliptically fibered Calabi-Yau threefolds, J. Alg. Geom. 12 (2003) 321 [math/0005196] [INSPIRE].

[91] V. Braun, T.W. Grimm and J. Keitel, Geometric engineering in toric F-theory and GUTs with U(1) gauge factors, JHEP 12 (2013) 069 [arXiv: 1306.0577] [INSPIRE].

[92] M.R. Douglas, R.L. Karp, S. Lukic and R. Reinbacher, Numerical solution to the hermitian Yang-Mills equation on the Fermat quintic, JHEP 12 (2007) 083 [hep-th/0606261] [INSPIRE].

[93] L.B. Anderson, V. Braun, R.L. Karp and B.A. Ovrut, Numerical Hermitian Yang-Mills connections and vector bundle stability in heterotic theories, JHEP 06 (2010) 107 [arXiv:1004 .4399] [INSPIRE].

[94] L.B. Anderson, V. Braun and B.A. Ovrut, Numerical Hermitian Yang-Mills connections and Kähler cone substructure, JHEP 01 (2012) 014 [arXiv:1103.3041] [INSPIRE].

[95] W.P. Li and Z. Qin, Donaldson-Thomas invariants of certain Calabi-Yau 3-folds, arXiv: 1002.4080 .

[96] W.P. Li and Z. Qin, Stable rank-2 bundles on Calabi-Yau manifolds, Internat. J. Math. 14 (2003) 1097.

[97] M. Lübke and A. Teleman, The Kobayashi-Hitchin correspondence, World Scientific, Singapore (1995).

[98] R. Hartshorne, Algebraic geometry, Graduate Texts in Mathematics, Springer, Germany (1977).

[99] L.B. Anderson, Heterotic and M-theory compactifications for string phenomenology, arXiv:0808.3621 [INSPIRE].

[100] R. Friedman and J. Morgan, Smooth four-manifolds and complex surfaces, Springer, New York U.S.A. (1994).

[101] L.B. Anderson, J.J. Heckman and S. Katz, T-branes and geometry, JHEP 05 (2014) 080 [arXiv:1310.1931] [INSPIRE].

[102] R. Donagi, Y.-H. He, B.A. Ovrut and R. Reinbacher, The particle spectrum of heterotic compactifications, JHEP 12 (2004) 054 [hep-th/0405014] [INSPIRE].

[103] G. Curio, Moduli restriction and chiral matter in heterotic string compactifications, JHEP 01 (2012) 015 [arXiv:1110.6315] [INSPIRE]. 
[104] S. Guerra, Spectral cover construction for associated bundles, Ph.D. dissertation, http://repository.upenn.edu/dissertations/AAI3260911, University of Pennsylvania, U.S.A. January 12007.

[105] L.B. Anderson, J. Gray, A. Lukas and B. Ovrut, The edge of supersymmetry: stability walls in heterotic theory, Phys. Lett. B 677 (2009) 190 [arXiv:0903.5088] [INSPIRE].

[106] L.B. Anderson, J. Gray, A. Lukas and B. Ovrut, Stability walls in heterotic theories, JHEP 09 (2009) 026 [arXiv:0905.1748] [INSPIRE].

[107] M. Atiyah, Vector bundles over an elliptic curve, Proc. London Math. Soc. 7 (1957) 414.

[108] J. Distler and B.R. Greene, Aspects of $(2,0)$ string compactifications, Nucl. Phys. B 304 (1988) 1 [INSPIRE].

[109] O. Okonek, M. Schneider and H. Spindler, Vector bundles on complex projective spaces, Birkhauser, Boston U.S.A. (1980).

[110] M. Bershadsky, T.M. Chiang, B.R. Greene, A. Johansen and C.I. Lazaroiu, F-theory and linear $\sigma$-models, Nucl. Phys. B 527 (1998) 531 [hep-th/9712023] [INSPIRE].

[111] R. Donagi, S. Katz and E. Sharpe, Spectra of D-branes with Higgs vevs, Adv. Theor. Math. Phys. 8 (2005) 813 [hep-th/0309270] [INSPIRE].

[112] S. Cecotti, C. Cordova, J.J. Heckman and C. Vafa, T-branes and monodromy, JHEP 07 (2011) 030 [arXiv: 1010.5780] [InSPIRE].

[113] R. Donagi and M. Wijnholt, Gluing branes, I, JHEP 05 (2013) 068 [arXiv:1104.2610] [INSPIRE].

[114] R. Donagi and M. Wijnholt, Gluing branes, II: flavour physics and string duality, JHEP 05 (2013) 092 [arXiv: 1112.4854] [inSPIRE].

[115] J. Marsano, N. Saulina and S. Schäfer-Nameki, Global gluing and G-flux, JHEP 08 (2013) 001 [arXiv: 1211.1097] [INSPIRE].

[116] A.P. Braun and T. Watari, On singular fibres in F-theory, JHEP 07 (2013) 031 [arXiv:1301.5814] [INSPIRE].

[117] R. Donagi, B.A. Ovrut and D. Waldram, Moduli spaces of five-branes on elliptic Calabi-Yau threefolds, JHEP 11 (1999) 030 [hep-th/9904054] [INSPIRE].

[118] B.A. Ovrut, T. Pantev and J. Park, Small instanton transitions in heterotic M-theory, JHEP 05 (2000) 045 [hep-th/0001133] [INSPIRE].

[119] E. Buchbinder, R. Donagi and B.A. Ovrut, Vector bundle moduli and small instanton transitions, JHEP 06 (2002) 054 [hep-th/0202084] [INSPIRE].

[120] T.W. Grimm, T.-W. Ha, A. Klemm and D. Klevers, Computing brane and flux superpotentials in F-theory compactifications, JHEP 04 (2010) 015 [arXiv:0909. 2025] [INSPIRE].

[121] T.W. Grimm, T.-W. Ha, A. Klemm and D. Klevers, Five-brane superpotentials and heterotic/F-theory duality, Nucl. Phys. B 838 (2010) 458 [arXiv:0912.3250] [INSPIRE].

[122] M. Bies, C. Mayrhofer, C. Pehle and T. Weigand, Chow groups, Deligne cohomology and massless matter in F-theory, arXiv:1402.5144 [INSPIRE].

[123] N.C. Bizet, A. Klemm and D.V. Lopes, Landscaping with fluxes and the $E_{8}$ Yukawa point in F-theory, arXiv: 1404.7645 [INSPIRE]. 
[124] B. Andreas and G. Curio, On discrete twist and four flux in $N=1$ heterotic/F-theory compactifications, Adv. Theor. Math. Phys. 3 (1999) 1325 [hep-th/9908193] [InSPIRE].

[125] P. Candelas, D.-E. Diaconescu, B. Florea, D.R. Morrison and G. Rajesh, Codimension three bundle singularities in F-theory, JHEP 06 (2002) 014 [hep-th/0009228] [INSPIRE].

[126] R. Donagi and M. Wijnholt, Model building with F-theory, Adv. Theor. Math. Phys. 15 (2011) 1237 [arXiv: 0802.2969] [INSPIRE].

[127] C. Beasley, J.J. Heckman and C. Vafa, GUTs and exceptional branes in F-theory - I, JHEP 01 (2009) 058 [arXiv:0802.3391] [inSPIRE].

[128] A.P. Braun, A. Collinucci and R. Valandro, G-flux in F-theory and algebraic cycles, Nucl. Phys. B 856 (2012) 129 [arXiv:1107.5337] [INSPIRE].

[129] J. Marsano and S. Schäfer-Nameki, Yukawas, G-flux and spectral covers from resolved Calabi-Yau's, JHEP 11 (2011) 098 [arXiv:1108.1794] [INSPIRE].

[130] S. Krause, C. Mayrhofer and T. Weigand, $G_{4}$ flux, chiral matter and singularity resolution in F-theory compactifications, Nucl. Phys. B 858 (2012) 1 [arXiv:1109.3454] [InSPIRE].

[131] T.W. Grimm and H. Hayashi, F-theory fluxes, chirality and Chern-Simons theories, JHEP 03 (2012) 027 [arXiv:1111.1232] [INSPIRE].

[132] H. Hayashi, T. Kawano, R. Tatar and T. Watari, Codimension-3 singularities and Yukawa couplings in F-theory, Nucl. Phys. B 823 (2009) 47 [arXiv:0901.4941] [InSPIRE].

[133] A. Collinucci and R. Savelli, On flux quantization in F-theory, JHEP 02 (2012) 015 [arXiv: 1011.6388] [INSPIRE].

[134] A. Collinucci and R. Savelli, On flux quantization in F-theory II: unitary and symplectic gauge groups, JHEP 08 (2012) 094 [arXiv:1203.4542] [INSPIRE].

[135] R. Donagi and E. Markman, Spectral curves, algebraically completely integrable Hamiltonian systems and moduli of bundles, alg-geom/9507017 [INSPIRE].

[136] R. Donagi, L. Ein and R. Lazarsfeld, Nilpotent cones and sheaves on K3 surfaces, Contemp. Math. 207 (1997) 51.

[137] P. Berglund and P. Mayr, Heterotic string/F-theory duality from mirror symmetry, Adv. Theor. Math. Phys. 2 (1999) 1307 [hep-th/9811217] [INSPIRE].

[138] R. Donagi and M. Wijnholt, Higgs bundles and UV completion in F-theory, Commun. Math. Phys. 326 (2014) 287 [arXiv:0904.1218] [INSPIRE].

[139] L.B. Anderson, J. Gray, A. Lukas and B. Ovrut, Stabilizing the complex structure in heterotic Calabi-Yau vacua, JHEP 02 (2011) 088 [arXiv:1010.0255] [INSPIRE].

[140] L.B. Anderson, J. Gray, A. Lukas and B. Ovrut, The Atiyah class and complex structure stabilization in heterotic Calabi-Yau compactifications, JHEP 10 (2011) 032 [arXiv:1107.5076] [INSPIRE].

[141] L.B. Anderson, J. Gray, A. Lukas and B. Ovrut, Vacuum varieties, holomorphic bundles and complex structure stabilization in heterotic theories, JHEP 07 (2013) 017 [arXiv: 1304.2704] [INSPIRE].

[142] G. Curio, Complete bundle moduli reduction in heterotic string compactifications, JHEP 05 (2012) 075 [arXiv: 1203.4961] [INSPIRE]. 
[143] K.S. Narain, New heterotic string theories in uncompactified dimensions $<10$, Phys. Lett. B 169 (1986) 41 [INSPIRE].

[144] P.H. Ginsparg, Comment on toroidal compactification of heterotic superstrings, Phys. Rev. D 35 (1987) 648 [INSPIRE].

[145] E. Witten, Small instantons in string theory, Nucl. Phys. B 460 (1996) 541 [hep-th/9511030] [INSPIRE].

[146] P.S. Aspinwall and M. Gross, The $\mathrm{SO}(32)$ heterotic string on a K3 surface, Phys. Lett. B 387 (1996) 735 [hep-th/9605131] [INSPIRE].

[147] K. Mohri, F-theory vacua in four-dimensions and toric threefolds, Int. J. Mod. Phys. A 14 (1999) 845 [hep-th/9701147] [INSPIRE].

[148] L.B. Anderson and W. Taylor, Geometric constraints in dual F-theory and heterotic string compactifications, arXiv:1405.2074 [INSPIRE].

[149] J.J. Heckman, Particle physics implications of F-theory, Ann. Rev. Nucl. Part. Sci. 60 (2010) 237 [arXiv: 1001.0577] [INSPIRE].

[150] T. Weigand, Lectures on F-theory compactifications and model building, Class. Quant. Grav. 27 (2010) 214004 [arXiv: 1009.3497] [INSPIRE].

[151] A. Maharana and E. Palti, Models of particle physics from Type IIB string theory and F-theory: a review, Int. J. Mod. Phys. A 28 (2013) 1330005 [arXiv:1212.0555] [InSPIRE].

[152] J. Marsano, N. Saulina and S. Schäfer-Nameki, F-theory compactifications for supersymmetric GUTs, JHEP 08 (2009) 030 [arXiv:0904.3932] [INSPIRE].

[153] J. Marsano, N. Saulina and S. Schäfer-Nameki, Monodromies, fluxes and compact three-generation F-theory GUTs, JHEP 08 (2009) 046 [arXiv:0906.4672] [INSPIRE].

[154] R. Blumenhagen, T.W. Grimm, B. Jurke and T. Weigand, Global F-theory GUTs, Nucl. Phys. B 829 (2010) 325 [arXiv: 0908.1784] [INSPIRE].

[155] T.W. Grimm, S. Krause and T. Weigand, F-theory GUT vacua on compact Calabi-Yau fourfolds, JHEP 07 (2010) 037 [arXiv: 0912.3524] [INSPIRE].

[156] M. Cvetič, I. Garcia-Etxebarria and J. Halverson, Global F-theory models: instantons and gauge dynamics, JHEP 01 (2011) 073 [arXiv: 1003.5337] [INSPIRE].

[157] C.-M. Chen, J. Knapp, M. Kreuzer and C. Mayrhofer, Global SO(10) F-theory GUTs, JHEP 10 (2010) 057 [arXiv: 1005.5735] [InSPIRE].

[158] J. Marsano, H. Clemens, T. Pantev, S. Raby and H.-H. Tseng, A global SU(5) F-theory model with Wilson line breaking, JHEP 01 (2013) 150 [arXiv:1206.6132] [INSPIRE].

[159] T.W. Grimm and T. Weigand, On Abelian gauge symmetries and proton decay in global F-theory GUTs, Phys. Rev. D 82 (2010) 086009 [arXiv:1006.0226] [INSPIRE].

[160] T.W. Grimm, M. Kerstan, E. Palti and T. Weigand, Massive Abelian gauge symmetries and fluxes in F-theory, JHEP 12 (2011) 004 [arXiv:1107.3842] [INSPIRE].

[161] D.S. Park and W. Taylor, Constraints on $6 D$ supergravity theories with Abelian gauge symmetry, JHEP 01 (2012) 141 [arXiv:1110.5916] [INSPIRE].

[162] D.S. Park, Anomaly equations and intersection theory, JHEP 01 (2012) 093 [arXiv:1111.2351] [INSPIRE]. 
[163] D.R. Morrison and D.S. Park, F-theory and the Mordell-Weil group of elliptically-fibered Calabi-Yau threefolds, JHEP 10 (2012) 128 [arXiv:1208.2695] [INSPIRE].

[164] M. Cvetič, T.W. Grimm and D. Klevers, Anomaly cancellation and Abelian gauge symmetries in F-theory, JHEP 02 (2013) 101 [arXiv:1210.6034] [INSPIRE].

[165] C. Mayrhofer, E. Palti and T. Weigand, U(1) symmetries in F-theory GUTs with multiple sections, JHEP 03 (2013) 098 [arXiv: 1211.6742] [INSPIRE].

[166] V. Braun, T.W. Grimm and J. Keitel, New global F-theory GUTs with U(1) symmetries, JHEP 09 (2013) 154 [arXiv: 1302.1854] [INSPIRE].

[167] J. Borchmann, C. Mayrhofer, E. Palti and T. Weigand, Elliptic fibrations for $\mathrm{SU}(5) \times \mathrm{U}(1) \times \mathrm{U}(1)$ F-theory vacua, Phys. Rev. D 88 (2013) 046005 [arXiv: 1303. 5054] [INSPIRE].

[168] M. Cvetič, D. Klevers and H. Piragua, F-theory compactifications with multiple U(1)-factors: constructing elliptic fibrations with rational sections, JHEP 06 (2013) 067 [arXiv:1303.6970] [INSPIRE].

[169] V. Braun, T.W. Grimm and J. Keitel, Geometric engineering in toric F-theory and GUTs with $\mathrm{U}(1)$ gauge factors, JHEP 12 (2013) 069 [arXiv: 1306. 0577] [INSPIRE].

[170] M. Cvetič, A. Grassi, D. Klevers and H. Piragua, Chiral four-dimensional F-theory compactifications with $\mathrm{SU}(5)$ and multiple $\mathrm{U}(1)$-factors, JHEP 04 (2014) 010 [arXiv:1306.3987] [INSPIRE].

[171] J. Borchmann, C. Mayrhofer, E. Palti and T. Weigand, SU(5) tops with multiple U(1)s in F-theory, Nucl. Phys. B 882 (2014) 1 [arXiv:1307.2902] [InSPIRE].

[172] M. Cvetič, D. Klevers, H. Piragua and P. Song, Elliptic fibrations with rank three Mordell-Weil group: F-theory with $\mathrm{U}(1) \times \mathrm{U}(1) \times \mathrm{U}(1)$ gauge symmetry, JHEP 03 (2014) 021 [arXiv: 1310.0463] [INSPIRE].

[173] A.P. Braun, A. Collinucci and R. Valandro, The fate of U(1)'s at strong coupling in F-theory, JHEP 07 (2014) 028 [arXiv: 1402.4054] [INSPIRE].

[174] M.R. Douglas, D.S. Park and C. Schnell, The Cremmer-Scherk mechanism in F-theory compactifications on K3 manifolds, JHEP 05 (2014) 135 [arXiv:1403.1595] [INSPIRE].

[175] J.J. Heckman, H. Lin and S.-T. Yau, Building blocks for generalized heterotic/F-theory duality, arXiv:1311.6477 [INSPIRE]. 